\title{
SOME ASPECTS OF ANALYSIS ON ALMOST COMPLEX MANIFOLDS WITH BOUNDARY
}

\author{
BERNARD COUPET, HERVÉ GAUSSIER AND ALEXANDRE SUKHOV
}

\begin{abstract}
We present some results dealing with the local geometry of almost complex manifolds. We establish mainly the complete hyperbolicity of strictly pseudoconvex domains, the extension of plurisubharmonic functions through generic submanifolds and the elliptic regularity of some diffeomorphisms in almost complex manifolds with boundary.
\end{abstract}

\section{Contents}

Introduction

1. Basic properties of almost complex structures

1.1. Almost complex structures

1.2. Pseudoholomorphic discs

1.3. Real submanifolds

1.4. Plurisubharmonic functions

1.5. Canonical almost complex bundles

2. Riemann mapping theorem for almost complex manifolds with boundary

2.1. Existence of discs attached to a real submanifold of an almost complex manifold

2.2. Generation of stationary discs

2.3. Canonical Foliation and the "Riemann map" associated with an almost complex structure

3. Kobayashi metric on almost complex manifolds

3.1. Localization of the Kobayashi-Royden metric

3.2. Uniform estimates of the Kobayashi-Royden metric

3.3. Boundary continuity and localization of biholomorphisms

4. Nonisotropic scaling of almost complex manifolds with boundary

4.1. Localization and boundary behavior of the tangent map

4.2. Non isotropic dilations

4.3. Lifts of biholomorphisms to the cotangent bundle : the case of arbitrary dimension

5. Elliptic regularity on almost complex manifolds with boundary

5.1. Reflection principle and regularity of analytic discs

5.2. Behavior of pseudoholomorphic maps near totally real submanifolds

5.3. Fefferman's mapping Theorem 


\section{INTRODUCTION}

Geometric invariants are natural objects in the problem of classifying varieties. For instance, the Riemann curvature is such an invariant in Riemannian geometry and isometries are the corresponding transformations. In symplectic geometry, the transformations are called symplectomorphisms but the Darboux Theorem states the non existence of local symplectic invariants.

In complex geometry, Cauchy-Riemann invariants are local invariants interferring in the classification of complex manifolds with boundaries. The generic corresponding situation is the equivalence problem between strictly pseudoconvex hypersurfaces in complex manifolds. Such classifications, initiated and completely treated by É.Cartan [13] in complex dimension two, was intensively studied and two important approaches in higher dimension are the theory of normal forms due to SS.Chern-J.Moser [16] and the Fefferman theorem [31] connecting complex and Cauchy-Riemann geometries.

The integral representation theory, which can be considered as the extension of the wellknown Cauchy-Green formula, was elaborated mainly by Grauert-Lieb [38] and Henkin [41] for strictly pseudoconvex domains in the Euclidean complex space and enabled to solve the $\bar{\partial}$-problem. In contrast with complex manifolds, there is generically no pseudoholomorphic map between almost complex manifolds, since the holomorphicity condition is given by a nonsolvable overdetermined system. In particular, the lack of holomorphic coordinates prevents from developing a straightforward integral representation theory.

The local existence of pseudoholomorphic discs in any almost complex manifold goes back to the work of N.Nijenhuis-W.Woolf [61]. With the idea of generalizing the NewlanderNirenberg Theorem to integrable almost complex manifolds with low regularity, they presented both an analytic and a geometric approach to the $\bar{\partial}_{J}$-equation, satisfied by pseudoholomorphic discs in an almost complex manifold $(M, J)$. This equation is locally a perturbation of the standard elliptic $\bar{\partial}$-equation; the local existence of pseudoholomorphic discs relies on the stability of the solutions to elliptic systems of partial differential equations under small quasi-linear elliptic deformation. Pseudoholomorphic discs played a fundamental rôle in symplectic geometry where some global symplectic invariants are associated to the moduli space of holomorphic curves for a compatible almost complex structure. Almost complex manifolds are viewed as natural manifolds for a deformation theory and pseudoholomorphic discs carry some information on the symplectic geometry by means of compactness phenomena.

The main purpose of this survey is to present some bases of local analysis in almost complex manifolds which consists in studying, essentially by analytic methods, local equivalence problems between strictly pseudoconvex domains. A large part of the survey is devoted to a precise study of pseudoholomorphic discs and of different objects whose existence is direcly issued from them. These are the Kobayashi-Royden metric and plurisubharmonic functions.

Most of our approach follows the concept of structures deformation. Nonisotropic dilations fitted to the geometry of strictly pseudoconvex hypersurfaces were introduced in complex analysis by S.Pinchuk [67] (related ideas were already used by Kuiper and Benzecri in the context of affine and projective geometry). The essential difference in almost complex manifolds relies on the non holomorphicity of the dilation maps. Hence the scaling procedure involves both a deformation of domains and of the ambiant almost complex structure. This 
general scheme drives to the presentation of some exotic non integrable almost complex structures, appearing as cluster points of dilated structures. These model almost complex structures imply new phenomena which distinguish almost complex manifolds from complex ones. A consequent part of our works relies on the study of these model structures.

This survey is for a large part an overview of different results obtained in a series of papers [33, 23, 24, 34]. This is organized as follows.

In Section 1, we mainly present the basic properties of almost complex manifolds. The first two subsections are devoted to generalities. In subsection 1.3, we explain how to attach pseudoholomorphic discs to totally real submanifolds and we introduce the model structures. These were introduced in [33]. We define complex hypersurfaces for these structures. In Subsection 1.4 we focus on plurisubharmonic functions. We first establish the Hopf Lemma and, as an application, we obtain the boundary distance preserving property for biholomorphisms between strictly pseudoconvex domains (proposition 1.4.8). Then we study the problem of removable singularities for plurisubharmonic functions. In Subsection 1.5 we present two constructions of almost complex structures on the tangent and on the cotangent bundles of an almost complex manifold. These canonical lifts play a major rôle in our studies and they will be used throughout the survey.

Section 2 concerns the study of stationary discs. The notion of stationary discs was introduced by L.Lempert [52] in the complex setting. Our results can be considered as a local analogue of Lempert's theory in almost complex manifolds. We prove the existence of stationary discs in the unit ball for small almost complex deformation of the standard complex structure (Propositions 2.2.1 and 2.2.2). We show that the stationary discs form a foliation of the unit ball, singular at the origin (Proposition 2.3.4). Then we define an analogue of the Riemann map and we give its main properties (Theorem 2.3.10). We end Section 2 with three applications : the boundary study of biholomorphisms (Corollary 2.3.11), a partial generalization of Cartan's theorem (Corollary 2.3.12) and the local biholomorphic equivalence problem between almost complex manifolds (Theorem 2.3.13).

Section 3 is devoted to the study of the Kobayashi metric in almost complex manifolds. Proving precise estimates of the Kobayashi-Royden metric in strictly pseudoconvex domains, similar to the estimates obtained by I.Graham [37] in the complex setting, we answer a question by S.Kobayashi about the existence of basis of complete hyperbolic neighborhoods at every point in an almost complex manifold. The general idea is to use a boundary localization principle (Proposition 3.1.5) and rescaling.

In Section 4 we study the boundary behaviour of a biholomorphism between two strictly pseudoconvex domains. We present the scaling procedure in details and we study the properties of the dilated objects. For a better understanding, we start with the case of four real dimensional almost complex manifolds in Subsections 4.1 and 4.2. One of the main results concerns the behaviour of the lift of a biholomorphism to the cotangent bundle presented in Proposition 4.2.6. The corresponding results in higher dimension are treated in Subsection 4.3. The analogue of Proposition 4.2.6 is Proposition 4.3.6. We end this Subsection with a compactness principle (Theorem 4.3.8). This can be considered as an almost complex analogue to the classical Wong-Rosay Theorem. 
Section 5 deals with the question of the regularity of pseudoholomorphic maps attached to totally real submanifolds in an almost complex manifold. These results are consequences of a geometric elliptic theory. In Subsection 5.1 we show that a pseudoholomorphic disc attached, in the sense of the cluster set, to a smooth totally real submanifold extends smoothly up to the boundary (Theorem 5.1.1). We point out that similar results have been established in the almost complex case under stronger assumptions on the initial boundary regularity of the disc. In Subsection 5.2 we establish the regularity of a pseudoholomorphic map, defined in a wedge attached to a totally real submanifold and whose image is contained, in the sense of the cluster set, into a totally real submanifold of an almost complex manifold (Proposition 5.2.1). As an application we obtain a partial version of the Fefferman Theorem (Corollary 5.2.4). Subsection 5.3 is devoted to the proof of the Fefferman Theorem, one of the main results of our survey (Theorem 5.3.1). This is a consequence of the results established in the previous sections.

\section{BAsiC PROPERTIES OF ALMOST COMPlEX STRUCTURES}

1.1. Almost complex structures. Everywhere in this paper $\Delta$ denotes the unit disc in $\mathbb{C}$ and $\mathbb{B}$ the unit ball in $\mathbb{C}^{n}$. Let $M$ be a smooth $\mathcal{C}^{\infty}$ real manifold of real dimension $2 n$.

Definition 1.1.1. (i) An almost complex structure on $M$ is a smooth $\mathcal{C}^{\infty}$-field $J$ on the tangent bundle $T M$ of $M$, satisfying $J^{2}=-I$.

(ii) If $J$ is an almost complex structure on $M$ then the 2-tuple $(M, J)$ is called an almost complex manifold.

By an abuse of notation $J_{s t}$ is the standard structure on $\mathbb{R}^{2 k}$ for every positive integer $k$. The standard structure $J_{s t}^{(2)}$ of $\mathbb{R}^{2}$ has the form

$$
J_{s t}^{(2)}=\left(\begin{array}{cc}
0 & -1 \\
1 & 0
\end{array}\right) .
$$

In $\mathbb{R}^{2 n}$ with standard coordinates $\left(x^{1}, y^{1}, \ldots, x^{n}, y^{n}\right)$ this is given by the block diagonal matrix :

$$
J_{s t}^{(2 n)}=\left(\begin{array}{cccc}
J_{s t}^{(2)} & & & \\
& J_{s t}^{(2)} & & \\
& & \cdot & \\
& & & J_{s t}^{(2)}
\end{array}\right) .
$$

In what follows we will just write $J_{s t}$ since the dimension of the space will be clear from the context. The first examples of almost complex manifolds are provided by complex manifolds. Namely, a complex manifold is a smooth real manifold $M$ of positive dimension $2 n$ with local complex analytic (holomorphic) charts $f=\left(f^{1}, \ldots, f^{n}\right)$ from $M$ to $\mathbb{C}^{n}$. The almost complex structure is locally defined by $J:=d f \circ J_{s t} \circ d f^{-1}$. We point out that $J$ does not depend on the choice of $f$. Thus an almost complex manifold $(M, J)$ is a complex manifold if for every point $p$ in $M$ there exists a neighborhood $U$ of $p$ and a coordinate diffeomorphism $z: U \rightarrow \mathbb{R}^{2 n}$ such that $d z \circ J \circ d z^{-1}=J_{\text {st }}$ on $z(U)$.

An important special case of an almost complex manifold is a bounded domain $D$ in $\mathbb{C}^{n}$ equipped with an almost complex structure $J$, defined in a neighborhood of $\bar{D}$, and 
sufficiently close to the standard structure $J_{s t}$ in the standard $\mathcal{C}^{k}$ norm on $\bar{D}$. Every almost complex manifold may be represented locally in such a form. More precisely, we have the following statement.

Lemma 1.1.2. Let $(M, J)$ be an almost complex manifold. Then for every point $p \in M$, every $\lambda_{0}>0$ and every real $k \geq 0$ there exist a neighborhood $U$ of $p$ and a coordinate diffeomorphism $z: U \rightarrow \mathbb{B}$ such that $z(p)=0, d z(p) \circ J(p) \circ d z^{-1}(0)=J_{\text {st }}$ and the direct image $z^{*}(J)=d z(p) \circ J(p) \circ d z^{-1}$ satisfies $\left\|z^{*}(J)-J_{s t}\right\|_{\mathcal{C}^{k}(\overline{\mathbb{B}})} \leq \lambda_{0}$.

Here $\mathcal{C}^{k}(\overline{\mathbb{B}})$ denotes the standard norm on $\mathbb{B}$

Proof. There exists a diffeomorphism $z$ from a neighborhood $U^{\prime}$ of $p \in M$ onto $\mathbb{B}$ satisfying $z(p)=0$ and $d z(p) \circ J(p) \circ d z^{-1}(0)=J_{s t}$. For $\lambda>0$ consider the dilation $d_{\lambda}: t \mapsto \lambda^{-1} t$ in $\mathbb{C}^{n}$ and the composition $z_{\lambda}=d_{\lambda} \circ z$. Then $\lim _{\lambda \rightarrow 0}\left\|z_{\lambda}^{*}(J)-J_{s t}\right\|_{\mathcal{C}^{2}(\bar{B})}=0$. Setting $U=z_{\lambda}^{-1}(\mathbb{B})$ for $\lambda>0$ small enough, we obtain the desired statement.

In particular, every almost complex structure $J$ sufficiently close to the standard structure $J_{s t}$ will be written locally $J=J_{s t}+\mathcal{O}(\|z\|)$. Finally by a small perturbation (or deformation) of the standard structure $J_{s t}$ defined in a neighborhood of $\bar{D}$, where $D$ is a domain in $\mathbb{C}^{n}$, we will mean a smooth one parameter family $\left(J_{\lambda}\right)_{\lambda}$ of almost complex structures defined in a neighborhood of $\bar{D}$, the real parameter $\lambda$ belonging to a neighborhood of the origin, and satisfying : $\lim _{\lambda \rightarrow 0}\left\|J_{\lambda}-J_{s t}\right\|_{\mathcal{C}^{k}(\bar{D})}=0$.

1.1.1. $\partial_{J}$ and $\bar{\partial}_{J}$ operators. Let $(M, J)$ be an almost complex manifold. We denote by $T M$ the real tangent bundle of $M$ and by $T_{\mathbb{C}} M:=\mathbb{C} \otimes T M$ its complexification. Recall that $T_{\mathbb{C}} M=T^{(1,0)} M \oplus T^{(0,1)} M$ where $T^{(1,0)} M:=\left\{X \in T_{\mathbb{C}} M: J X=i X\right\}=\{\zeta-i J \zeta, \zeta \in T M\}$, and $T^{(0,1)} M:=\left\{X \in T_{\mathbb{C}} M: J X=-i X\right\}=\{\zeta+i J \zeta, \zeta \in T M\}$. Let $T^{*} M$ denote the cotangent bundle of $M$. Identifying $\mathbb{C} \otimes T^{*} M$ with $T_{\mathbb{C}}^{*} M:=\operatorname{Hom}\left(T_{\mathbb{C}} M, \mathbb{C}\right)$ we define the set of complex forms of type $(1,0)$ on $M$ by : $T_{(1,0)}^{*} M=\left\{w \in T_{\mathbb{C}}^{*} M: w(X)=0, \forall X \in T^{(0,1)} M\right\}$ and the set of complex forms of type $(0,1)$ on $M$ by : $T_{(0,1)}^{*} M=\left\{w \in T_{\mathbb{C}}^{*} M: w(X)=\right.$ $\left.0, \forall X \in T^{(1,0)} M\right\}$. Then $T_{\mathbb{C}}^{*} M=T_{(1,0)}^{*} M \oplus T_{(0,1)}^{*} M$.

This allows to define the operators $\partial_{J}$ and $\bar{\partial}_{J}$ on the space of smooth functions defined on $M$ : given a complex smooth function $u$ on $M$, we set $\partial_{J} u=d u_{(1,0)} \in T_{(1,0)}^{*} M$ and $\bar{\partial}_{J} u=d u_{(0,1)} \in T_{(0,1)}^{*} M$. As usual, differential forms of any bidegree $(p, q)$ on $(M, J)$ are defined by means of the exterior product.

We point out that there is a one-to-one correspondence between almost complex structures and independent complex one forms. More precisely, to any almost complex structure one can associate a basis $\left(w^{1}, \ldots, \omega^{n}\right)$ of $(1,0)$-forms. The conjugated forms $\bar{\omega}^{1}, \ldots, \bar{\omega}^{n}$ define a basis of $T_{(0,1)}^{*} M$ and $w^{1}, \ldots, \omega^{n}, \bar{\omega}^{1}, \ldots, \bar{\omega}^{n}$ are $\mathbb{C}$-linearly independent. Conversely, if $n$ complex one forms $\omega^{1}, \ldots, \omega^{n}$ on $T_{\mathbb{C}}^{*} M$ are such that $w^{1}, \ldots, \omega^{n}, \bar{\omega}^{1}, \ldots, \bar{\omega}^{n}$ are $\mathbb{C}$-linearly independent, one can define an almost complex structure on $M$ by asserting that $\left(\omega^{1}, \ldots, \omega^{n}\right)$ is a basis of $T_{(1,0)}^{*} M$. The corresponding almost complex structure $J$ is defined as follows. Set $\omega^{j}=\zeta^{j}+i \eta^{j}$. The one forms $\zeta^{1}, \ldots, \zeta^{n}, \eta^{1}, \ldots, \eta^{n}$ define a basis of $T M$. Then define $J$ on $T M$ by $J \zeta^{j}=\eta^{j}$ for $j=1, \ldots, n$. 
1.1.2. Integrability. Let $\left(X_{\overline{1}}, \ldots, X_{\bar{n}}\right)$ be a basis of $T_{(0,1)}^{*} M$. If $M$ is a complex manifold, then one can find local charts $f=\left(f^{1}, \ldots, f^{n}\right)$ such that

$$
X_{\bar{j}} f^{k}=0 \text { for every } j, k=1, \ldots, n .
$$

Equation (1.1) is equivalent to the equation $d f^{j}\left(X_{\bar{j}}\right)=0$ for $j, k=1, \ldots, n$, meaning that $\left(d f^{1}, \ldots, d f^{n}\right)$ form a basis of $T_{(1,0)}^{*} M$. As a direct consequence of (1.1) we have $\left[X_{\bar{j}}, X_{\bar{k}}\right]=0$ for every $j, k=1, \ldots, n$. This last condition is equivalent to the integrability of the two fiber bundles $T^{(1,0)} M$ and $T^{(0,1)} M$. One can rewrite the integrability of $T^{(1,0)} M$ as

$$
N_{J}(\zeta, \eta)=0 \text { for every } \zeta, \eta \in T M
$$

where $N_{J}$ is the Nijenhuis tensor defined on $T M \times T M$ by :

$$
N_{J}(\zeta, \eta)=[J \zeta, J \eta]-J[J \zeta, \eta]-J[\zeta, J \eta]-[\zeta, \eta]
$$

The content of the Newlander-Nirenberg theorem is the following (see [60, 40, 81]) :

Theorem 1.1.3. An almost complex manifold $(M, J)$ is a complex manifold if and only if $T^{(1,0)} M$ is integrable.

We point out that the integrability condition can also be interpreted in terms of forms as follows : $(M, J)$ is a complex manifold if and only if $d \omega$ has no $(0,2)$ component for every $(1,0)$ form $\omega$.

Let $J$ be an almost complex structure defined in a neighborhood of the origin in $\mathbb{R}^{2 n}$ endowed with the usual standard complex coordinates $z^{1}, \ldots, z^{n}$. We assume that $J(0)=J_{s t}$. Since $\left(d z^{1}, \ldots, d z^{n}\right)$ form a basis of $(1,0)$ forms at the origin, one can find a basis of $(1,0)$ forms $\left(\omega^{1}, \ldots, \omega^{n}\right)$ such that $\omega^{j}(0)=d z^{j}$ for $j=1, \ldots, n$. Hence one can assume that $\omega^{j}=d z^{j}+\sum_{k=1} A_{j}^{j}(z, \bar{z}) d \bar{z}^{k}$, where $A_{k}^{j}$ are smooth functions satisfying $A_{k}^{j}(0,0)=0$. One cannot impose the condition $\left(\partial A_{k}^{j} / \partial \bar{z}\right)(0)=0$ unless the structure $J$ is integrable at the origin. However, if ... then by the change of variables ... one can add the normalization condition $\left(\partial A_{k}^{j} / \partial z\right)(0)=0$. This normalization will be used in the local description of strictly pseudoconvex hypersurfaces, see Subsection 1.3.

We point out that ny almost complex structure on a real surface is integrable.

Below in this first section we will describe some examples of almost complex structures which will play essential role thoughhout the paper. These are model almost complex structures (defined and studied in Subsection 1.3) and canonical almost complex structures on the tangent and cotangent bundles (Subsection 1.5). These different structures will be the center of our study in the forecoming Sections.

1.2. Pseudoholomorphic discs. A smooth map $f$ between two almost complex manifolds $\left(M^{\prime}, J^{\prime}\right)$ and $(M, J)$ is holomorphic if its differential satisfies the following holomorphicity condition :

$$
d f \circ J=J^{\prime} \circ d f \text { on } T M^{\prime} .
$$


Lemma 1.2.1. The map $f$ is $\left(J^{\prime}, J\right)$ holomorphic if and only if

$$
\forall \omega \in T_{(1,0)}^{*} M^{\prime}, f^{*} w \in T_{(1,0)}^{*} M .
$$

Here $f^{*} w$ is the complex one form defined on $T_{\mathbb{C}} M$ by $f^{*} \omega=\omega \circ d f$.

Proof. System (1.3) implies that $d f(X) \in T^{(0,1)} M$ for every $X \in T^{(0,1)} M^{\prime}$. In particular, if $\omega \in T_{(1,0)}^{*} M$ and $X \in T^{(0,1)} M^{\prime}$ then $\left(f^{*} \omega\right)(X)=\omega(d f(X))=0$. Conversely, assume that condition (1.4) is satisfied. If $X \in T^{(0,1)} M^{\prime}$ and $\omega \in T_{(1,0)}^{*} M$, then $\omega(d f(X))=0$. Hence, $d f(X)_{i} n T^{(0,1)} M$ and $d f(J X)=-i d f(X)=J^{\prime}(d f(X))$. One can prove similarly the equality $d f \circ J=J^{\prime} \circ d f$ on $T^{(1,0)} M^{\prime}$, implying system (1.3).

Generically, if $\operatorname{dim}_{\mathbb{R}} M^{\prime}=2 k>2$ then system (1.3) is overdetermined. If $k=1$ it follows from the previous subsection that $J^{\prime}$ is integrable. In particular, one can view locally $\left(M^{\prime}, J^{\prime}\right)$ as $\left(\Delta, J_{s t}\right)$. In case $\left(M^{\prime}, J^{\prime}\right)=\left(\Delta, J_{s t}\right)$ the map $f$ is called a $J$-holomorphic disc. We denote by $\zeta$ the complex variable in $\mathbb{C}: \zeta=x+i y$. Since $J_{s t}(\partial / \partial x)=\partial / \partial y$ system (1.3) can be written :

or equivalently

$$
\frac{\partial f}{\partial y}=J(f) \frac{\partial f}{\partial x}
$$

$$
\left(J+J_{s t}\right) \frac{\partial f}{\partial \bar{\zeta}}=\left(J-J_{s t}\right) \frac{\partial f}{\partial \zeta} .
$$

In view of Lemma 1.1.2, $J+J_{\text {st }}$ is locally invertible. Then the holomorphicity condition is usually written as

$$
\frac{\partial f}{\partial \bar{\zeta}}+Q_{J}(f) \frac{\partial f}{\partial \zeta}=0
$$

where $Q_{J}$ is an endomorphism of $\mathbb{R}^{2 n}$ given by $Q_{J}=\left(J_{s t}+J\right)^{-1}\left(J_{s t}-J\right)$. However it is easy to see that $Q_{J}$ is an anti $\mathbb{C}$-linear endomorphism of $\mathbb{C}^{n}$. One can locally write a basis $w:=\left(w^{1}, \ldots, w^{n}\right)$ of $(1,0)$ forms on $M$ as $w^{j}=d z^{j}+\sum_{k=1}^{n} A_{k}^{j}(z, \bar{z}) d \bar{z}$ where $A_{k}^{j}$ is a smooth function satisfying the normalization conditions $A_{k}^{j}(0,0)=0$. According to condition (1.4) the disc $f$ being $J$-holomorphic if $f^{*}\left(w^{j}\right)$ is a $(1,0)$ form for $j=1, \ldots, n$ (see [18]), meaning that $f^{*}\left(w^{j}\right)(\partial / \partial \bar{\zeta})=0, f$ satisfies the following equation on $\Delta$ :

$$
\frac{\partial f}{\partial \bar{\zeta}}+A(f) \frac{\overline{\partial f}}{\partial \zeta}=0
$$

where $A=\left(A_{j, k}\right)_{1 \leq j, k \leq n}$. We will use equation (1.5) to characterize the $J$-holomorphicity in the survey.

The Nijenhuis-Woolf theorem gives the existence of pseudoholomorphic discs and their smooth dependence on initial data. The following general form is due to S.Ivashkovich and J.P.Rosay.

Proposition 1.2.2. Let $k \in \mathbb{N}, k \geq 1$, and $0<\alpha<1$. Let $(M, J)$ be an almost complex manifold with $J$ of class $\mathcal{C}^{k, \alpha}$ and let $p \in M$. Then for every sufficiently small $V=\left(v_{1}, \ldots, v_{k}\right) \in \mathcal{J}_{p}^{k} M$, there exists a $\mathcal{C}^{k+1, \alpha} J$-holomorphic map $u_{p, V}$ from $\Delta$ into $M$ such that the $k^{\text {th }}$ jet of $u_{p, V}$ at the origin is equal to $(p, V)$. Moreover, $u_{p, V}$ can be chosen with $\mathcal{C}^{1}$ dependence (in $\mathcal{C}^{k, \alpha}$ ) on the parameters $(p, V)$ in $\mathcal{J}^{k} M$. 
Here $\mathcal{J}^{k} M$ denotes the space of jets of order $k$ of maps from the unit disc $\Delta$ to $M$.

Proof. We follow the proof given in [44]. If we fix a chart $B$ containing $p$, we can assume that $p=0 \in \mathbb{R}^{2 n}$ and $J(0)=J_{s t}$. Hence there exists a neighborhood $U$ of 0 such that equation (1.5) is defined for every disc $u$, defined on $\Delta$, with values in $U$.

Consider the Cauchy-Green operator $T_{C G}$ for maps $g$ continuous on $\bar{\Delta}$, with values in a complex vector space :

$$
\forall \zeta \in \bar{\Delta}, T_{C G}(g)(\zeta)=\frac{1}{2 \pi i} \iint_{\Delta} \frac{g(\tau)}{\zeta-\tau} d \tau \overline{d \tau} .
$$

The operator $T_{C G}$ satisfies the following properties :

(a) $g \in \mathcal{C}^{k, \alpha}(\bar{\Delta}) \Rightarrow T_{C G} g \in \mathcal{C}^{k+1, \alpha}(\bar{\Delta}), \forall k \in \mathbb{N}, 0<\alpha<1$,

(b) $\frac{\partial}{\partial \bar{\zeta}}\left(T_{C G}(g)\right)=g$ on $\bar{\Delta}$.

By considering $A_{t}(u):=A(t u)$ and $u_{t, \tau}(\zeta):=t^{-1} u(\tau \zeta)$ we can assume that $\|A\|_{\mathcal{C}^{1}}$ is small enough. Moreover, in view of equation (1.5), the $J$-holomorphicity of a disc $u$ is equivalent to the (usual) holomorphicity of the disc $h=\left(I d-T_{C G}\left(A(u) \overline{\frac{\partial}{\partial \zeta}}\right)\right) u$. Consider now the $\mathcal{C}^{k}$ mapping :

$$
\begin{array}{rlr}
\Phi:(-1,1) \times \mathcal{C}^{k}(\Delta, B) & \rightarrow & \mathcal{C}^{k}\left(\Delta, \mathbb{C}^{n}\right) \\
(t, u) & \mapsto\left(I d-T_{C G}\left(A(t u) \overline{\frac{\partial}{\partial \zeta}}\right)\right) u .
\end{array}
$$

Since $\Phi(0, u)=u$, it follows from the Implicit Function Theorem that there exists $0<t_{0}<1$ such that for $|t|<t_{0}$ the map $\Phi(t,$.$) is a \mathcal{C}^{k}$ diffeomorphism from a neighborhood of the origin in $\mathcal{C}^{k}(\Delta, B)$ onto a neighborhood of the origin $V$ in $\mathcal{C}^{k}\left(\Delta, \mathbb{C}^{n}\right)$.

For $w=\left(w_{1}, \ldots, w_{k}\right) \in\left(\mathbb{C}^{n}\right)^{k}$ small enough, the holomorphic map $h_{q, w}$ defined on $\bar{\Delta}$ by

$$
h_{q, w}(\zeta)=\sum_{l=1}^{k} \frac{1}{l !} \zeta^{l} w_{l}
$$

belongs to $V$. If $u_{t, w}:=\Phi(t, .)^{-1}\left(h_{q, w}\right)$ then $t u_{t, w}$ is $J$-holomorphic. Moreover, since $u_{0, w}=$ $h_{w}$, the $k-t h$ jet of $u_{0, w}$ at the origin is equal to $w$. hence, for sufficiently small positive $t$ the map $w \mapsto\left(\frac{\partial u_{t, w}}{\partial \operatorname{Re}(\zeta)}(0), \ldots, \frac{\partial^{k} u_{t, w}}{\partial\left(\operatorname{Re}(\zeta)^{k}\right)}(0)\right)$ is a diffeomorphism between neighborhoods of the origin in $\left(\mathbb{C}^{n}\right)^{k}$.

Remark 1.2.3. The statement of Proposition 1.2 .2 means that there exists a on-to-one correpondence between sufficiently small $J$-holomorphic discs and standard holomorphic discs.

The following Proposition establishes the stability of arbitrary $J$-holomorphic discs under perturbation of the center and of the derivative at the center. This result due to IvashkovichRosay in [44] is fundamental for the upper semi-continuity of the Kobayashi Royden pseudonorm.

Proposition 1.2.4. Let $(M, J)$ be an almost complex manifold with $J$ of class $\mathcal{C}^{1, \alpha}(\alpha>0)$. Let $u$ be a $J$-holomorphic map from a neighborhood of $\bar{\Delta}$ into $M$. There exists a neighborhood $V$ of $\left(u(0), \frac{\partial u}{\partial \operatorname{Re}(\zeta)}(0)\right)$ in $T M$ such that for every $(q, X) \in V$, there exists a J-holomorphic map $v: \Delta \rightarrow M$ with $v(0)=q, \frac{\partial v}{\partial \operatorname{Rer}(\zeta)}(0)=x$. 
Proof. We follow the exposition of [44]. Assume that $u$ is defined on $\Delta_{r}$ for some $r>1$. Since the map $\tilde{u}$ is $J_{s t} \times J$ - holomorphic from $\Delta_{r}$ into $\mathbb{R}^{2} \times M$, it is sufficient to prove Proposition 1.2 .4 for imbedded discs. Moreover, there exist $(n-1)$ smooth vector fields $Y_{1}, \ldots, Y_{n-1}$, defined in a neighborhood of $u\left(\Delta_{r}\right)$ such that for every $\zeta \in \Delta_{r}$, the vectors $\frac{\partial u}{\partial x}(\zeta), Y_{1}(\zeta), \ldots, Y_{n-1}(\zeta)$ are $J(u(z))$-linearly independent. Consider now the $\mathcal{C}^{2, \alpha}$ change of variables $\Phi$

$$
\left(z_{1}, \ldots, z_{n}\right) \mapsto u\left(z_{1}\right)+\sum_{j=1}^{n-1} z_{j+1} Y_{j}\left(u\left(z_{1}\right)\right)
$$

defined for $\left|z_{1}\right|<r,\left|z_{j}\right|$ small if $j \geq 2$. The structure $\Phi^{*}(J)$ is a $\mathcal{C}^{1, \alpha}$ almost complex structure that coincides with the standard structure on $\mathbb{C} \times\{0\} \subset \mathbb{C}^{n}$. So Proposition 1.2.4 reduces to the following Lemma:

Lemma 1.2.5. Let $J$ be a $\mathcal{C}^{1, \alpha}$ almost complex structure on $\mathbb{R}^{2 n}$ that coincides with the standard complex structure on $\mathbb{C} \times\{0\}$. Let $U$ be a neighborhood of $\bar{\Delta} \times\{0\}$. For any $(q, t) \in \mathbb{C}^{n} \times \mathbb{C}^{n}$ close enough to $(0,0)$, there exists a J-holomorphic map $v: \Delta \rightarrow U$ such that $v(0)=q$ and $\frac{\partial v}{\partial \operatorname{Re}(\zeta)}(0)=(1,0, \ldots, 0)+t$.

Proof of Lemma 1.2.5. Consider a neighborhood of $\bar{\Delta} \times\{0\}$ on which the $J$-holomorphicity condition can be written :

$$
\frac{\partial u}{\partial \bar{\zeta}}+A_{J}(u) \frac{\overline{\partial u}}{\partial \zeta}=0
$$

with $A_{J}(\zeta, 0, \ldots, 0)=0$.

Set $\mathcal{E}_{0}=\left\{f: \bar{\Delta} \rightarrow \mathbb{C}^{n} / f \in \mathcal{C}^{1, \alpha}, f(0)=0, \nabla f(0)=0\right\}, \mathcal{F}_{0}=\left\{g: \bar{\Delta} \rightarrow \mathbb{C}^{n} / g \in \mathcal{C}^{\alpha}, g(0)=\right.$ $0\}$ and $F(z)=(z, 0, \ldots, 0)$.

Define the map

$$
\begin{aligned}
\Psi: \mathcal{E}_{0} & \rightarrow \mathcal{F}_{0} \\
f & \mapsto \frac{\partial(F+f)}{\partial \bar{\zeta}}+A_{J}(F+f) \frac{\overline{\partial(F+f)}}{\partial \zeta}
\end{aligned}
$$

Since $\frac{\partial F}{\partial \bar{\zeta}}+0$ and $A_{J}(F)=0$ we have :

$$
\Psi(f)=\frac{\partial f}{\partial \bar{\zeta}}+B_{J}(f) \frac{\overline{\partial F}}{\partial \zeta}+o(|f|)
$$

where $B_{J}$ is a $(2 n \times 2 n)$ matrix with $\mathcal{C}^{\alpha}$ entries in $z$ and $\mathbb{R}$-linear in $f$. We want to show that the derivative $D \Psi_{0}$ of the map $\Psi$ at $f=0$ is onto.

In complex notations we can write :

$$
D \Psi_{0}(f)(\zeta)=\frac{\partial f}{\partial \bar{\zeta}}(\zeta)+B_{1}(\zeta) f(\zeta)+B_{2}(\zeta) \overline{f(\zeta)}
$$

where $B_{1}$ and $B_{2}$ are $(n \times n)$ complex matrices with $\mathcal{C}^{\alpha}$ coefficients.

The surjectivity of $D \Psi_{0}$ follows from the following classical result (see [44] for a direct proof) 
Lemma 1.2.6. If $B_{1}, B_{2}$ are $(n \times n)$ complex matrices with $\mathcal{C}^{\alpha}$ coefficients on $\bar{\Delta}$, for every $g \in \mathcal{F}_{0}$, there exists $f \in \mathcal{E}_{0}$ such that

$$
\frac{\partial f}{\partial \bar{\zeta}}(\zeta)+B_{1}(\zeta) f(\zeta)+B_{2}(\zeta) \overline{f(\zeta)}=g(\zeta)
$$

for every $\zeta \in \Delta$.

1.3. Real submanifolds. This Subsection deals with the study of totally real and of strictly pseudoconvex submanifolds in an almost complex manifold. In the first part, we attach Bishop's discs to a totally real submanifold. In the second part, we describe locally strictly pseudoconvex hypersurfaces in real dimension four as deformations of strictly pseudoconvex hypersurfaces for the standard structure. The associated scaling procedure is our main tool for the study of strictly pseudoconvex domains in almost complex manifolds.

Let $\Gamma$ be a real smooth submanifold in $M$ and let $p \in \Gamma$. We denote by $H^{J}(\Gamma)$ the $J$-holomorphic tangent bundle $T \Gamma \cap J T \Gamma$.

Definition 1.3.1. The real submanifold $\Gamma$ is called totally real if $H^{J}(\Gamma)=\{0\}$ and is called $J$-complex if $H^{J}(\Gamma)=T \Gamma$.

We note that if $\Gamma$ is a real hypersurface in $M$ defined by $\Gamma=\{r=0\}$ and $p \in \Gamma$ then by definition $H_{p}^{J}(\Gamma)=\left\{v \in T_{p} M: d r(p)(v)=d r(p)(J(p) v)=0\right\}$.

As usual, if $\theta$ is a one form on $M$ then $J^{*} \theta$ is the form acting on a vector field $X$ by $\left(J^{*} \theta\right) X=\theta(J X)$.

We recall the notion of the Levi form of a hypersurface :

Definition 1.3.2. Let $\Gamma=\{r=0\}$ be a smooth real hypersurface in $M$ ( $r$ is any smooth defining function of $\Gamma$ ) and let $p \in \Gamma$.

(i) The Levi form of $\Gamma$ at $p$ is the map defined on $H_{p}^{J}(\Gamma)$ by $\mathcal{L}_{\Gamma}^{J}\left(X_{p}\right)=J^{\star} d r[X, J X]_{p}$, where the vector field $X$ is any section of the J-holomorphic tangent bundle $H^{J} \Gamma$ such that $X(p)=X_{p}$.

(ii) A real smooth hypersurface $\Gamma=\{r=0\}$ in $M$ is strictly $J$-pseudoconvex at $p$ if $\mathcal{L}_{\Gamma}^{J}\left(X_{p}\right)>0$ for any nonzero $X_{p} \in H_{p}^{J}(\Gamma)$. The hypersurface $\Gamma$ is called strictly $J_{-}$ pseudoconvex if it is strictly $J$-pseudoconvex at every point.

Remark 1.3.3. ( $i$ ) the "strict $J$-pseudoconvexity" condition does not depend on the choice of a smooth defining function of $\Gamma$. Indeed if $\rho$ is an other smooth defining function for $\Gamma$ in a neighborhood of $p \in \Gamma$ then there exists a positive smooth function $\lambda$ defined in a neighborhood of $p$ such that $\rho=\lambda r$. In particular $\left(J^{\star} d r\right)(p)=\lambda(p)\left(J^{\star} d \rho\right)(p)$.

(ii) since the map $(r, J) \mapsto J^{\star} d r$ is smooth the "strict $J$-pseudoconvexity" is stable under small perturbations of both the hypersurface and the almost complex structure.

Let $X \in T M$. It follows from the identity $d\left(J^{\star} d r\right)(X, J X)=X\left(<J^{\star} d r, J X>\right)-J X(<$ $\left.J^{\star} d r, X>\right)-\left(J^{\star} d r\right)[X, J X]$ that $\left(J^{\star} d r\right)[X, J X]=-d\left(J^{\star} d r\right)(X, J X)$ for every $X \in H^{J} \Gamma$, since $<d r, J X>=<d r, J X>=0$ in that case. Hence we set 
Definition 1.3.4. Let $p \in M$. If $r$ is a $\mathcal{C}^{2}$ function on $M$ then the Levi form of $r$ at $p$ is defined on $T_{p} M$ by $\mathcal{L}_{r}{ }^{J}(p, v):=-d\left(J^{\star} d r\right)_{p}(X, J X)$ where $X$ is any section of $T M$ such that $X(p)=v$.

1.3.1. Almost complex perturbation of discs. In this subsection we attach Bishop's discs to a totally real submanifold in an almost complex manifold. The following statement is an almost complex analogue of the well-known Pinchuk's construction [65] of a family of holomorphic discs attached to a totally real manifold.

Lemma 1.3.5. For any $\delta>0$ there exists a family of $J$-holomorphic discs $h(\tau, t)=h_{t}(\tau)$ smoothly depending on the parameter $t \in \mathbb{R}^{2 n}$ such that $h_{t}\left(\partial \Delta^{+}\right) \subset E, h_{t}(\Delta) \subset W(\Omega, E)$, $W_{\delta}(\Omega, E) \subset \cup_{t} h_{t}(\Delta)$ and $C_{1}(1-|\tau|) \leq \operatorname{dist}\left(h_{t}(\tau), E\right) \leq C_{2}(1-|\tau|)$ for any $t$ and any $\tau \in \Delta^{+}$, with constants $C_{j}>0$ independent of $t$.

For $\alpha>1$, noninteger, we denote by $\mathcal{C}^{\alpha}(\bar{\Delta})$ the Banach space of functions of class $\mathcal{C}^{\alpha}$ on $\bar{\Delta}$ and by $\mathcal{A}^{\alpha}$ the Banach subspace of $\mathcal{C}^{\alpha}(\bar{\Delta})$ of functions holomorphic on $\Delta$.

First we consider the situation where $E=\left\{r:=\left(r_{1}, \ldots, r_{n}\right)=0\right\}$ is a smooth totally real submanifold in $\mathbb{C}^{n}$. Let $J_{\lambda}$ be an almost complex deformation of the standard structure $J_{s t}$ that is a one-parameter family of almost complex structures so that $J_{0}=J_{s t}$. We recall that for $\lambda$ small enough the $\left(J_{s t}, J_{\lambda}\right)$-holomorphicity condition for a map $f: \Delta \rightarrow \mathbb{C}^{n}$ may be written in the form

$$
\bar{\partial}_{J_{\lambda}} f=\bar{\partial} f+q(\lambda, f) \overline{\partial f}=0
$$

where $q$ is a smooth matrix satisfying $q(0, \cdot) \equiv 0$, uniquely determined by $J_{\lambda}([73])$.

A disc $f \in\left(\mathcal{C}^{\alpha}(\bar{\Delta})\right)^{n}$ is attached to $E$ and is $J_{\lambda}$-holomorphic if and only if it satisfies the following nonlinear boundary Riemann-Hilbert type problem :

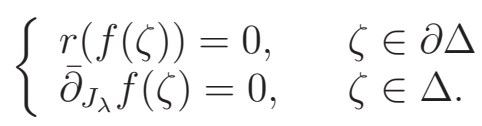

Let $f^{0} \in\left(\mathcal{A}^{\alpha}\right)^{n}$ be a disc attached to $E$ and let $\mathcal{U}$ be a neighborhood of $\left(f^{0}, 0\right)$ in the space $\left(\mathcal{C}^{\alpha}(\bar{\Delta})\right)^{n} \times \mathbb{R}$. Given $(f, \lambda)$ in $\mathcal{U}$ define the maps $v_{f}: \zeta \in \partial \Delta \mapsto r(f(\zeta))$ and

$$
\begin{aligned}
& u: \mathcal{U} \rightarrow\left(\mathcal{C}^{\alpha}(\partial \Delta)\right)^{n} \times \mathcal{C}^{\alpha-1}(\Delta) \\
& (f, \lambda) \mapsto\left(v_{f}, \bar{\partial}_{J_{\lambda}} f\right) .
\end{aligned}
$$

Denote by $X$ the Banach space $\left(\mathcal{C}^{\alpha}(\bar{\Delta})\right)^{n}$. Since $r$ is of class $\mathcal{C}^{\infty}$, the map $u$ is smooth and the tangent map $D_{X} u\left(f^{0}, 0\right)$ (we consider the derivative with respect to the space $X$ ) is a linear map from $X$ to $\left(\mathcal{C}^{\alpha}(\partial \Delta)\right)^{n} \times \mathcal{C}^{\alpha-1}(\Delta)$, defined for every $h \in X$ by

$$
D_{X} u\left(f^{0}, 0\right)(h)=\left(\begin{array}{l}
2 R e[G h] \\
\bar{\partial}_{J_{0}} h
\end{array}\right),
$$

where for $\zeta \in \partial \Delta$

$$
G(\zeta)=\left(\begin{array}{lll}
\frac{\partial r_{1}}{\partial z^{1}}\left(f^{0}(\zeta)\right) & \cdots & \frac{\partial r_{1}}{\partial z^{n}}\left(f^{0}(\zeta)\right) \\
\cdots & \cdots & \cdots \\
\frac{\partial r_{n}}{\partial z^{1}}\left(f^{0}(\zeta)\right) & \cdots & \frac{\partial r_{n}}{\partial z^{n}}\left(f^{0}(\zeta)\right)
\end{array}\right)
$$


(see [35]).

Lemma 1.3.6. Assume that for some $\alpha>1$ the linear map from $\left(\mathcal{A}^{\alpha}\right)^{n}$ to $\left(\mathcal{C}^{\alpha-1}(\Delta)\right)^{n}$ given by $h \mapsto 2 R e[G h]$ is surjective and has a d-dimensional kernel. Then there exist $\delta_{0}, \lambda_{0}>0$ such that for every $0 \leq \lambda \leq \lambda_{0}$, the set of $J_{\lambda}$-holomorphic discs $f$ attached to $E$ and such that $\left\|f-f^{0}\right\|_{\alpha} \leq \delta_{0}$ forms a smooth d-dimensional submanifold $\mathcal{A}_{\lambda}$ in the Banach space $\left(C^{\alpha}(\bar{\Delta})\right)^{n}$.

Proof of Lemma 1.3.6. According to the implicit function Theorem, the proof of Lemma 1.3.6 reduces to the proof of the surjectivity of $D_{X} u$. It follows by classical one-variable results on the resolution of the $\bar{\partial}$-problem in the unit disc that the linear map from $X$ to $\mathcal{C}^{\alpha-1}(\Delta)$ given by $h \mapsto \bar{\partial} h$ is surjective. More precisely, given $g \in \mathcal{C}^{\alpha-1}(\Delta)$ consider the Cauchy transform

$$
T_{C G}(g): \tau \in \partial \Delta \mapsto \frac{1}{2 \pi i} \iint_{\Delta} \frac{g(\zeta)}{\zeta-\tau} d \zeta \wedge d \bar{\zeta} .
$$

For every function $g \in C^{\alpha-1}(\Delta)$ the solutions $h \in X$ of the equation $\bar{\partial} h=g$ have the form $h=h_{0}+T_{\Delta}(g)$ where $h_{0}$ is an arbitrary function in $\left(\mathcal{A}^{\alpha}\right)^{n}$. Consider the equation

$$
D_{X} u\left(f^{0}, 0\right)(h)=\left(\begin{array}{l}
g_{1} \\
g_{2}
\end{array}\right)
$$

where $\left(g_{1}, g_{2}\right)$ is a vector-valued function with components $g_{1} \in \mathcal{C}^{\alpha-1}(\partial \Delta)$ and $g_{2} \in \mathcal{C}^{\alpha-1}(\Delta)$. Solving the $\bar{\partial}$-equation for the second component, we reduce equation (1.7) to

$$
2 \operatorname{Re}\left[G(\zeta) h_{0}(\zeta)\right]=g_{1}-2 \operatorname{Re}\left[G(\zeta) T_{\Delta}\left(g_{2}\right)(\zeta)\right]
$$

with respect to $h_{0} \in\left(\mathcal{A}^{\alpha}\right)^{n}$. The surjectivity of the map $h_{0} \mapsto 2 R e\left[G h_{0}\right]$ gives the result.

Proof of Lemma1.3.5. We proceed in three steps.

Step 1. Filling the polydisc. Consider the $n$-dimensional real torus $\mathbb{T}^{n}=\partial \Delta \times \ldots \times \partial \Delta$ in $\mathbb{C}^{n}$ and the linear disc $f^{0}(\zeta)=(\zeta, \ldots, \zeta), \zeta \in \Delta$ attached to $\mathbb{T}^{n}$. In that case, a disc $h^{0}$ is in the kernel of $h \mapsto 2 R e[G h]$ if and only if every component $h_{k}^{0}$ of $h^{0}$ satisfies on $\partial \Delta$ the condition $h_{k}^{0}+\zeta^{2} \overline{h_{k}^{0}}=0$. Considering the Fourier expansion of $h_{k}$ on $\partial \Delta$ (recall that $h_{k}$ is holomorphic on $\Delta$ ) and identifying the coefficients, we obtain that the map $h \mapsto 2 R e[G h]$ from $\left(\mathcal{A}^{\alpha}\right)^{n}$ to $\left(C^{\alpha-1}(\Delta)\right)^{n}$ is surjective and has a $3 n$-dimensional kernel. By Lemma 1.3.6 if $J_{\lambda}$ is an almost complex structure close enough to $J_{s t}$ in a neighborhood of the closure of the polydisc $\Delta^{n}$, there is a $3 n$-parameters family of $J_{\lambda}$-holomorphic discs attached to $\mathbb{T}^{n}$. These $J_{\lambda}$-holomorphic discs fill the intersection of a sufficiently small neighborhood of the point $(1, \ldots, 1)$ with $\Delta^{n}$.

Step 2. Isotropic dilations. Consider a smooth totally real submanifold $E$ in an almost complex manifold $(M, J)$. Fixing local coordinates, we may assume that $E$ is a submanifold in a neighborhood of the origin in $\mathbb{C}^{n}, J=J_{s t}+0(|z|)$ and $E$ is defined by the equations $y=\phi(x)$, where $\nabla \phi(0)=0$. For every $\varepsilon>0$, consider the isotropic dilations $\Lambda_{\varepsilon}: z \mapsto$ $z^{\prime}=\varepsilon^{-1} z$. Then $J_{\varepsilon}:=\Lambda_{\varepsilon}(J) \rightarrow J_{s t}$ as $\varepsilon \rightarrow 0$. In the $z^{\prime}$-coordinates $E$ is defined by the equations $y^{\prime}=\psi\left(x^{\prime}, \varepsilon\right):=\varepsilon^{-1} \phi\left(\varepsilon x^{\prime}\right)$ and $\psi \rightarrow 0$ as $\varepsilon \rightarrow 0$. Consider the local diffeomorphism $\Phi_{\varepsilon}: z^{\prime}=x^{\prime}+i y^{\prime} \mapsto z^{\prime \prime}=x^{\prime}+i\left(y^{\prime}-\psi\left(x^{\prime}, \varepsilon\right)\right)$. Then in new coordinates (we omit the primes) $E$ coincides with a neighborhood of the origin in $\mathbb{R}^{n}=\{y=0\}$ and $\hat{J}_{\varepsilon}:=\left(\Phi_{\varepsilon}\right)_{*}\left(J_{\varepsilon}\right) \rightarrow J_{s t}$ as 
$\varepsilon \rightarrow 0$. Assume for instance that $E=(]-1,1[\times\{0\})^{n}$. For $j=1, \ldots, n$, let $\Gamma_{j}$ be a smooth simple curve in the real plane $\left\{\left(x_{j}, y_{j}\right): x_{j} \leq 0\right\}$, containing $]-1 / 2,1 / 2[$ and bounding a domain $G_{j}$. If $\psi_{j}$ is a $J_{s t}$-biholomorphism from $G_{j}$ to $\Delta$ then the map $\psi:=\left(\psi_{1}, \ldots, \psi_{n}\right)$ is a $J_{s t}$-biholomorphism from $G_{1} \times \cdots \times G_{n}$ to $\Delta^{n}$. Hence we may assume that $E$ is a neighborhood of the point $(1, \ldots, 1)$ on the torus $\mathbb{T}^{n}$ and the almost complex structure $J_{\varepsilon}$ is a small deformation of the standard structure in a neighborhood of $\bar{\Delta}^{n}$. By Step 1, we may fill a neighborhood of the point $(1, \ldots, 1)$ in the polydisc $\Delta^{n}$ by $J_{\varepsilon}$-holomorphic discs (for $\varepsilon$ small enough) which are small perturbations of the disc $\zeta \mapsto(\zeta, \ldots, \zeta)$. Returning to the initial coordinates, we obtain a family of $J$-holomorphic discs attached to $E$ along a fixed arc (say, the upper semicircle $\partial \Delta^{+}$) and filling the intersection of a neighborhood of the origin with the wedge $\{y-\phi(x)<0\}$.

Step3. Let now $W(\Omega, E)=\left\{r_{j}<0, j=1, \ldots, n\right\}$ be a wedge with edge $E$; we assume that $0 \in E$ and $J(0)=J_{s t}$. We may assume that $E=\{y=\phi(x)\}, \nabla \phi(0)=0$, since the linear part of every $r_{j}$ at the origin is equal to $y_{j}$. So shrinking $\Omega$ if necessary, we obtain that for any $\delta>0$ the wedge $W_{\delta}(\Omega, E)=\left\{z \in \Omega: r_{j}(z)-\delta \sum_{k \neq j} r_{k}(z)<0, j=1, \ldots, n\right\}$ is contained in the wedge $\{z \in \Omega: y-\phi(x)<0\}$. By Step 2 there is a family of $J$-holomorphic discs attached to $E$ along the upper semicircle and filling the wedge $W_{\delta}(\Omega, E)$. These discs are smooth up to the boundary and smoothly depend on the parameters.

1.3.2. Local description of strictly pseudoconvex domains. If $\Gamma$ is a germ of a real hypersurface in $\mathbb{C}^{n}$ strictly pseudoconvex with respect to $J_{s t}$, then $\Gamma$ remains strictly pseudoconvex for any almost complex structure $J$ sufficiently close to $J_{s t}$ in the $\mathcal{C}^{2}$-norm. Conversely a strictly pseudoconvex hypersurface in an almost complex manifold of real dimension four can be represented, in suitable local coordinates, as a strictly $J_{s t}$-pseudoconvex hypersurface equipped with a small deformation of the standard structure. Indeed, according to [73] Corollary 3.1.2, there exist a neighborhood $U$ of $q$ in $M$ and complex coordinates $z=\left(z^{1}, z^{2}\right): U \rightarrow B \subset \mathbb{C}^{2}$, $z(q)=0$ such that $z_{*}(J)(0)=J_{s t}$ and moreover, a map $f: \Delta \rightarrow B$ is $J^{\prime}:=z_{*}(J)$-holomorphic if it satisfies the equations

$$
\frac{\partial f^{j}}{\partial \bar{\zeta}}=A_{j}\left(f^{1}, f^{2}\right) \overline{\left(\frac{\partial f^{j}}{\partial \zeta}\right)}, j=1,2
$$

where $A_{j}(z)=O(|z|), j=1,2$.

To obtain such coordinates, one can consider two transversal foliations of the ball $\mathbb{B}$ by $J^{\prime}$-holomorphic curves (see [61]) and then take these curves into the lines $z^{j}=$ const by a local diffeomorphism (see Figure 1).

The direct image of the almost complex structure $J$ under such a diffeomorphism has a diagonal matrix $J^{\prime}\left(z^{1}, z^{2}\right)=\left(a_{j k}(z)\right)_{j k}$ with $a_{12}=a_{21}=0$ and $a_{j j}=i+\alpha_{j j}$ where $\alpha_{j j}(z)=\mathcal{O}(|z|)$ for $j=1,2$. We point out that the lines $z^{j}=$ const are $J$-holomorphic after a suitable parametrization (which, in general, is not linear). 

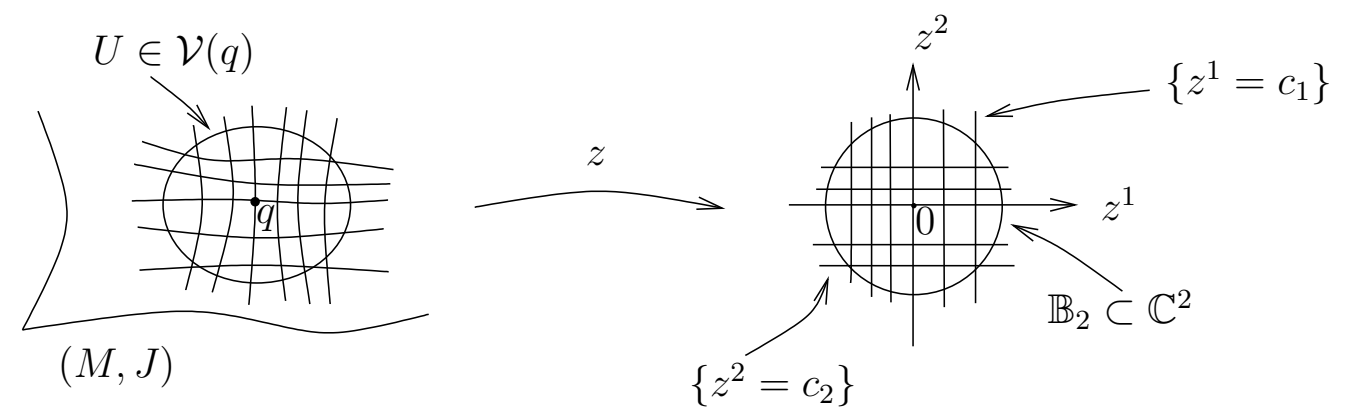

Figure 1

In what follows we omit the prime and denote this structure again by $J$. We may assume that the complex tangent space $T_{0}(\partial D) \cap J(0) T_{0}(\partial D)=T_{0}(\partial D) \cap i T_{0}(\partial D)$ is given by $\left\{z^{2}=0\right\}$. In particular, we have the following expansion for the defining function $\rho$ of $D$ on $U: \rho(z, \bar{z})=2 \operatorname{Re}\left(z^{2}\right)+2 \operatorname{Re} K(z)+H(z)+\mathcal{O}\left(|z|^{3}\right)$, where $K(z)=\sum k_{\nu \mu} z^{\nu} z^{\mu}, k_{\nu \mu}=k_{\mu \nu}$ and $H(z)=\sum h_{\nu \mu} z^{\nu} \bar{z}^{\mu}, h_{\nu \mu}=\bar{h}_{\mu \nu}$.

Lemma 1.3.7. The domain $D$ is strictly $J_{s t}$-pseudoconvex near the origin.

Proof of Lemma 1.3.7. Consider a complex vector $v=\left(v_{1}, 0\right)$ tangent to $\partial D$ at the origin. Let $f: \Delta \rightarrow \mathbb{C}^{2}$ be a $J$-holomorphic disc centered at the origin and tangent to $v: f(\zeta)=$ $v \zeta+\mathcal{O}\left(|\zeta|^{2}\right)$. Since $A_{2}=\mathcal{O}(|z|)$, it follows from the $J$-holomorphicity equation (1.8) that $\left(f^{2}\right)_{\zeta \bar{\zeta}}(0)=0$. This implies that $(\rho \circ f)_{\zeta \bar{\zeta}}(0)=H(v)$. Thus, the Levi form with respect to $J$ coincides with the Levi form with respect to $J_{s t}$ on the complex tangent space of $\partial D$ at the origin. This proves Lemma 1.3.7.

Consider the non-isotropic dilations $\Lambda_{\delta}:\left(z^{1}, z^{2}\right) \mapsto\left(\delta^{-1 / 2} z^{1}, \delta^{-1} z^{2}\right)=\left(w^{1}, w^{2}\right)$ with $\delta>0$. If $J$ has the above diagonal form in the coordinates $\left(z^{1}, z^{2}\right)$ in $\mathbb{C}^{2}$, then its direct image $J_{\delta}=\left(\Lambda_{\delta}\right)_{*}(J)$ has the form $J_{\delta}\left(w^{1}, w^{2}\right)=\left(a_{j k}\left(\delta^{1 / 2} w^{1}, \delta w^{2}\right)\right)_{j k}$ and so $J_{\delta}$ tends to $J_{s t}$ in the $\mathcal{C}^{2}$ norm as $\delta \rightarrow 0$. On the other hand, $\partial D$ is, in the $w$ coordinates, the zero set of the function $\rho_{\delta}=\delta^{-1}\left(\rho \circ \Lambda_{\delta}^{-1}\right)$. As $\delta \rightarrow 0$, the function $\rho_{\delta}$ tends to the function $2 R e w^{2}+2 R e K\left(w^{1}, 0\right)+$ $H\left(w^{1}, 0\right)$ which defines a strictly $J_{s t}$-pseudoconvex domain by Lemma 1.3 .7 and proves the claim.

This also proves that if $\rho$ is a local defining function of a strictly $J$-pseudoconvex domain, then $\tilde{\rho}:=\rho+C \rho^{2}$ is a strictly $J$-plurisubharmonic function, quite similarly to the standard case.

In conclusion we point out that extending $\tilde{\rho}$ by a suitable negative constant, we obtain that if $D$ is a strictly $J$-pseudoconvex domain in an almost complex manifold, then there exists a neighborhood $U$ of $\bar{D}$ and a function $\rho, J$-plurisubharmonic on $U$ and strictly $J$ plurisubharmonic in a neighborhood of $\partial D$, such that $D=\{\rho<0\}$.

1.3.3. Model almost complex structures. The scaling process in complex manifolds deals with deformations of domains under holomorphic transformations called dilations. The usual nonisotropic dilations in complex manifolds, associated with strictly pseudoconvex domains, 
provide the unit ball (after biholomorphism) as the limit domain. In almost complex manifolds dilations are generically no more holomorphic with respect to the ambiant structure. The scaling process consists in deforming both the structure and the domain. This provides, as limits, a quadratic domain and a linear deformation of the standard structure in $\mathbb{R}^{2 n}$, called model structure. We study some invariants of such structures. Let $\left(x^{1}, y^{1}, \ldots, x^{n}, y^{n}\right)=\left(z^{1}, \ldots, z^{n}\right)=\left({ }^{\prime} z, z^{n}\right)$ denote the canonical coordinates of $\mathbb{R}^{2 n}$.

Definition 1.3.8. Let $J$ be an almost complex structure on $\mathbb{C}^{n}$. We call $J$ a model structure if $J(z)=J_{s t}+L(z)$ where $L$ is given by a linear matrix $L=\left(L_{j, k}\right)_{1 \leq j, k \leq 2 n}$ such that $L_{j, k}=0$ for $1 \leq j \leq 2 n-2,1 \leq k \leq 2 n, L_{j, k}=0$ for $j, k=2 n-1,2 n$ and $L_{j, k}=\sum_{l=1}^{n-1}\left(a_{l}^{j, k} z^{l}+\bar{a}_{l}^{j, k} \bar{z}^{l}\right)$, $a_{l}^{j, k} \in \mathbb{C}$, for $j=2 n-1,2 n$ and $k=1, \cdots, 2 n-2$.

The complexification $J_{\mathbb{C}}$ of a model structure $J$ can be written as a $(2 n \times 2 n)$ complex matrix

$$
J_{\mathbb{C}}=\left(\begin{array}{ccccccc}
i & 0 & 0 & 0 & \cdots & 0 & 0 \\
0 & -i & 0 & 0 & \cdots & 0 & 0 \\
0 & 0 & i & 0 & \cdots & 0 & 0 \\
0 & 0 & 0 & -i & \cdots & 0 & 0 \\
\cdots & \cdots & \cdots & \cdots & \cdots & \cdots & \cdots \\
0 & \tilde{L}_{2 n-1,2} & 0 & \tilde{L}_{2 n-1,4} & \cdots & i & 0 \\
\tilde{L}_{2 n, 1} & 0 & \tilde{L}_{2 n, 3} & 0 & \cdots & 0 & -i
\end{array}\right)
$$

where $\tilde{L}_{2 n-1,2 k}(z, \bar{z})=\sum_{l=1, l \neq k}^{n-1}\left(\alpha_{l}^{k} z^{l}+\beta_{l}^{k} \bar{z}^{l}\right)$ with $\alpha_{l}^{k}, \beta_{l}^{k} \in \mathbb{C}$. Moreover, $\tilde{L}_{2 n, 2 k-1}=\overline{\tilde{L}_{2 n-1,2 k}}$.

With a model structure we associate model domains.

Definition 1.3.9. Let $J$ be a model structure on $\mathbb{C}^{n}$ and $D=\left\{z \in \mathbb{C}^{n}: R e z^{n}+P_{2}\left({ }^{\prime} z{ }^{\prime} \bar{z}\right)<\right.$ $0\}$, where $P_{2}$ is homogeneous second degree real polynomial on $\mathbb{C}^{n-1}$. The pair $(D, J)$ is called a model domain if $D$ is strictly J-pseudoconvex in a neighborhood of the origin.

The aim of this Section is to define the complex hypersurfaces for model structures in $\mathbb{R}^{2 n}$. Let $J$ be a model structure on $\mathbb{R}^{2 n}$ and let $N$ be a germ of a $J$-complex hypersurface in $\mathbb{R}^{2 n}$.

\section{Proposition 1.3.10.}

(i) The model structure $J$ is integrable if and only if $\tilde{L}_{2 n-1, j}$ satisfies the compatibility conditions

for every $1 \leq j, k \leq n-1$.

$$
\frac{\partial \tilde{L}_{2 n-1,2 k}}{\partial \bar{z}^{j}}=\frac{\partial \tilde{L}_{2 n-1,2 j}}{\partial \bar{z}^{k}}
$$

In that case there exists a global diffeomorphism of $\mathbb{R}^{2 n}$ which is $\left(J, J_{\text {st }}\right)$ holomorphic. In that case the germs of any J-complex hypersurface are given by one of the two following forms :

(a) $N=A \times \mathbb{C}$ where $A$ is a germ of a $J_{\text {st }}$-complex hypersurface in $\mathbb{C}^{n-1}$,

(b) $N=\left\{\left({ }^{\prime} z, z^{n}\right) \in \mathbb{C}^{n}: z^{n}=\frac{i}{4} \sum_{j=1}^{n-1} \bar{z}^{j} \tilde{L}_{2 n-1,2 j}\left({ }^{\prime} z,^{\prime} \bar{z}\right)+\frac{i}{4} \sum_{j=1}^{n-1} \bar{z}^{j} \tilde{L}_{2 n-1,2 j}\left({ }^{\prime} z, 0\right)\right)+$ $\left.\tilde{\varphi}\left({ }^{\prime} z\right)\right\}$ where $\tilde{\varphi}$ is a holomorphic function locally defined in $\mathbb{C}^{n-1}$. 
(ii) If $J$ is not integrable then $N=A \times \mathbb{C}$ where $A$ is a germ of a $J_{\text {st }}$-complex hypersurface in $\mathbb{C}^{n-1}$.

Proof of Proposition 1.3.10 Let $N$ be a germ of a $J$-complex hypersurface in $\mathbb{R}^{2 n}$. If $\pi$ : $\mathbb{R}^{2 n} \rightarrow \mathbb{R}^{2 n-2}$ is the projection on the $(2 n-2)$ first variables, it follows from Definition 1.3.8, or similarly from condition (1.9) that $\pi\left(T_{z} N\right)$ is a $J_{s t}$-complex hypersurface in $\mathbb{C}^{n-1}$.

It follows that either $\operatorname{dim}_{\mathbb{C}} \pi(N)=n-1$ or $\operatorname{dim}_{\mathbb{C}} \pi(N)=n-2$.

Case one : $\operatorname{dim}_{\mathbb{C}} \pi(N)=n-1$. We prove the following Lemma :

Lemma 1.3.11. There is a local holomorphic function $\tilde{\varphi}$ in $\mathbb{C}^{n-1}$ such that $N=\left\{\left({ }^{\prime} z, z^{n}\right)\right.$ : $\left.\left.z^{n}=\frac{i}{4} \sum_{j=1}^{n-1} \bar{z}^{j} \tilde{L}_{2 n-1,2 j}\left({ }^{\prime} z,{ }^{\prime} \bar{z}\right)+\frac{i}{4} \sum_{j=1}^{n-1} \bar{z}^{j} \tilde{L}_{2 n-1,2 j}\left({ }^{\prime} z, 0\right)\right)+\tilde{\varphi}\left({ }^{\prime} z\right)\right\}$.

Proof of Lemma 1.3.11. A germ $N$ can be represented as a graph $N=\left\{z^{n}=\varphi\left({ }^{\prime} z,^{\prime} \bar{z}\right)\right\}$ where $\varphi$ is a smooth local complex function. Hence $T_{z} N=\left\{v_{n}=\sum_{j=1}^{n-1}\left(\frac{\partial \varphi}{\partial z^{j}}\left({ }^{\prime} z\right) v_{j}+\right.\right.$ $\left.\left.\frac{\partial \varphi}{\partial \bar{z}^{j}}\left({ }^{\prime} z\right) \bar{v}_{j}\right)\right\}$. A vector $v=\left(x^{1}, y^{1}, \ldots, x^{n}, y^{n}\right)$ belongs to $T_{z} N$ if and only if the complex components $v^{1}:=x^{1}+i y^{1}, \ldots, v^{n}:=x^{n}+i y^{n}$ satisfy

$$
i v_{n}=i \sum_{j=1}^{n-1}\left(\frac{\partial \varphi}{\partial z^{j}}\left({ }^{\prime} z\right) v_{j}+\frac{\partial \varphi}{\partial \bar{z}^{j}}\left({ }^{\prime} z\right) \bar{v}_{j}\right) .
$$

Similarly, the vector $J_{z} v$ belongs to $T_{z} N$ if and only if

$$
\sum_{j=1}^{n-1} \tilde{L}_{2 n, 2 j-1}\left({ }^{\prime} z\right) \bar{v}_{j}+i v_{n}=i\left(\sum_{j=1}^{n-1} \frac{\partial \varphi}{\partial z^{j}}\left({ }^{\prime} z\right) v_{j}-\sum_{j=1}^{n-1} \frac{\partial \varphi}{\partial \bar{z}^{j}}\left({ }^{\prime} z\right) \bar{v}_{j}\right) .
$$

It follows from (1.10) and (1.11) that $N$ is $J$-complex if and only if

$$
\sum_{j=1}^{n-1}\left(\tilde{L}_{2 n, 2 j-1}\left({ }^{\prime} z\right) \bar{v}_{j}+2 i \frac{\partial \varphi}{\partial \bar{z}^{j}}(z) \bar{v}_{j}\right)=0
$$

for every ${ }^{\prime} v \in \mathbb{C}^{n-1}$, or equivalently if and only if

$$
\tilde{L}_{2 n, 2 j-1}=-2 i \frac{\partial \varphi}{\partial \bar{z}^{j}}
$$

for every $j=1, \cdots, n-1$. This last condition is equivalent to the compatibility conditions

$$
\frac{\partial \tilde{L}_{2 n, 2 j-1}}{\partial \bar{z}^{k}}=\frac{\partial \tilde{L}_{2 n, 2 k-1}}{\partial \bar{z}^{j}} \text { for } j, k=1, \cdots, n-1 .
$$

In that case there exists a local holomorphic function $\tilde{\varphi}$ in $\mathbb{C}^{n-1}$ such that

$$
\varphi\left({ }^{\prime} z,^{\prime} \bar{z}\right)=\frac{i}{2} \sum_{j=1}^{n-1} \bar{z}^{j}\left(\sum_{k \neq j} \alpha_{k}^{j} z^{k}\right)-\frac{i}{2} \sum_{j=1}^{n-2} \bar{z}^{j}\left(\sum_{k>j} \beta_{k}^{j} \bar{z}^{k}\right)+\tilde{\varphi}\left({ }^{\prime} z\right),
$$

meaning that such $J$-complex hypersurfaces are parametrized by holomorphic functions in the variables ' $z$. Moreover we can rewrite $\varphi$ as

$$
\left.\varphi\left({ }^{\prime} z,^{\prime} \bar{z}\right)=\frac{i}{4} \sum_{j=1}^{n-1} \bar{z}^{j} \tilde{L}_{2 n-1,2 j}\left({ }^{\prime} z,{ }^{\prime} \bar{z}\right)+\frac{i}{4} \sum_{j=1}^{n-1} \bar{z}^{j} \tilde{L}_{2 n-1,2 j}\left({ }^{\prime} z, 0\right)\right)+\tilde{\varphi}\left({ }^{\prime} z\right) .
$$


We also have the following

Lemma 1.3.12. The $(1,0)$ forms of $J$ have the form $\omega=\sum_{k=1}^{n} c_{k} d z^{k}-\frac{i}{2} c_{n} \sum_{k=1}^{n-1} \tilde{L}_{2 n-1,2 k} d \bar{z}^{k}$ with complex numbers $c_{1}, \ldots, c_{n}$.

Proof of Lemma 1.3.12 Let $X=\sum_{k=1}^{n}\left(x^{k} \frac{\partial}{\partial z^{k}}+y^{k} \frac{\partial}{\partial \bar{z}^{k}}\right)$ be a $(0,1)$ vector field. In view of (1.9), we have :

$$
J_{\mathbb{C}}(X)=-i X \Leftrightarrow\left\{\begin{array}{l}
x^{k}=0, \text { for } \mathrm{k}=1, \ldots, \mathrm{n}-1 \\
x^{n}=\frac{i}{2} \sum_{k=1}^{n-1} y_{k} \tilde{L}_{2 n-1,2 k} .
\end{array}\right.
$$

Hence the $(0,1)$ vector fields are given by

$$
X=\sum_{k=1}^{n} y^{k} \frac{\partial}{\partial \bar{z}^{k}}+\frac{i}{2} \frac{\partial}{\partial z^{n}} \sum_{k=1}^{n-1} y^{k} \tilde{L}_{2 n-1,2 k}
$$

A $(1,0)$ form $\omega=\sum_{k=1}^{n}\left(c_{k} d z^{k}+d_{n} d \bar{z}^{k}\right)$ satisfying $\omega(X)=0$ for every $(0,1)$ vector field $X$ it satisfies $d_{n}=0$ and $d_{k}+(i / 2) c_{n} \tilde{L}_{2 n-1,2 k}=0$ for every $k=1, \ldots, n-1$. This gives the desired form for the $(1,0)$ forms on $\mathbb{C}^{n}$.

Consider now the global diffeomorphism of $\mathbb{C}^{n}$ defined by

$$
F\left({ }^{\prime} z, z^{n}\right)=\left({ }^{\prime} z, z^{n}-\frac{i}{4} \sum_{j=1}^{n-1} \bar{z}^{j} \tilde{L}_{2 n-1,2 j}\left({ }^{\prime} z{ }^{\prime} \bar{z}\right)-\frac{i}{4} \sum_{j=1}^{n-1} \bar{z}^{j} \tilde{L}_{2 n-1,2 j}\left({ }^{\prime} z, 0\right)\right)
$$

The map $F$ is $\left(J, J_{s t}\right)$ holomorphic if and only if $F^{*}\left(d z^{k}\right)$ is a $(1,0)$ form with respect to $J$, for every $k=1, \ldots, n$.

Then $F^{*}\left(d z^{k}\right)=d z^{k}$ for $k=1, \ldots, n-1$ and

$$
\begin{aligned}
F^{*}\left(d z^{n}\right)= & d z^{n}+\sum_{k=1}^{n-1} \frac{\partial F_{n}}{\partial z^{k}} d z^{k}+\sum_{k=1}^{n-1} \frac{\partial F_{n}}{\partial \bar{z}^{k}} d \bar{z}^{k} \\
= & d z^{n}+\sum_{k=1}^{n-1} \frac{\partial F_{n}}{\partial z^{k}} d z^{k} \\
& -\frac{i}{4} \sum_{k=1}^{n-1}\left(\tilde{L}_{2 n-1,2 k}\left({ }^{\prime} z,{ }^{\prime} \bar{z}\right)+\sum_{j \neq k} \bar{z}^{j} \frac{\partial \tilde{L}_{2 n-1,2 j}}{\partial \bar{z}^{k}}\left({ }^{\prime} z,^{\prime} \bar{z}\right)+\tilde{L}_{2 n-1,2 k}\left({ }^{\prime} z,^{\prime} 0\right) d \bar{z}^{k} .\right.
\end{aligned}
$$


By the compatibility condition (1.12) we have

$$
\begin{aligned}
F^{*}\left(d z^{n}\right)= & d z^{n}+\sum_{k=1}^{n-1} \frac{\partial F_{n}}{\partial z^{k}} d z^{k} \\
& -\frac{i}{4} \sum_{k=1}^{n-1}\left(\tilde{L}_{2 n-1,2 k}\left({ }^{\prime} z,^{\prime} \bar{z}\right)+\tilde{L}_{2 n-1,2 k}\left({ }^{\prime} 0,^{\prime} \bar{z}\right)+\tilde{L}_{2 n-1,2 k}\left({ }^{\prime} z,^{\prime} 0\right)\right) d \bar{z}^{k} \\
= & d z^{n}-\frac{i}{2} \sum_{k=1}^{n-1} \tilde{L}_{2 n-1,2 k}\left({ }^{\prime} z,^{\prime} \bar{z}\right) d \bar{z}^{k}+\sum_{k=1}^{n-1} \frac{\partial F_{n}}{\partial z^{k}} d z^{k} .
\end{aligned}
$$

These equalities mean that $F$ is a local $\left(J, J_{s t}\right)$-biholomorphism of $\mathbb{C}^{n}$, and so that $J$ is integrable.

Case two : $\operatorname{dim}_{\mathbb{C}} \pi(N)=n-2$. In that case we may write $N=\pi(N) \times \mathbb{C}$, meaning that $J$-complex hypersurfaces are parametrized by $J_{s t}$-complex hypersurfaces of $\mathbb{C}^{n-1}$.

We can conclude now the proof of Proposition 1.3.10, We proved in Case one that if there exists a $J$-complex hypersurface in $\mathbb{C}^{n}$ such that $\operatorname{dim} \pi(N)=n-1$ (this is equivalent to the compatibility conditions (1.12) ) then $J$ is integrable. Conversely, it is immediate that if $J$ is integrable then there exists a $J$-complex hypersurface whose form is given by Lemma 1.3.11 and hence that the compatibility conditions (1.12) are satisfied. This gives part $(i)$ of Proposition 1.3.10.

To prove part $(i i)$, we note that if $J$ is not integrable then in view of part $(i)$ the form of any $J$-complex hypersurface is given by Case two.

1.4. Plurisubharmonic functions. In this Subsection, we present essentially two results. The first establishes the Hopf Lemma. As a consequence, we obtain the boundary equivalence property for biholomorphisms between relatively compact, strictly pseudoconvex domains. The second result deals with the removability of singularities for plurisubharmonic functions.

We first recall the following definition :

Definition 1.4.1. An upper semicontinuous function $u$ on $(M, J)$ is called $J$ plurisubharmonic on $M$ if the composition $u \circ f$ is subharmonic on $\Delta$ for every J-holomorphic $\operatorname{disc} f: \Delta \rightarrow M$.

If $M$ is a domain in $\mathbb{C}^{n}$ and $J=J_{s t}$ then a $J_{s t}$-plurisubharmonic function is a plurisubharmonic function in the usual sense.

Proposition 1.4.2. Let $r$ be a real function of class $C^{2}$ in a neighborhood of a point $p \in M$.

(i) If $F:(M, J) \longrightarrow\left(M^{\prime}, J^{\prime}\right)$ is a $\left(J, J^{\prime}\right)$-holomorphic map, and $\varphi$ is a real function of class $C^{2}$ in a neighborhood of $F(p)$, then for any $v \in T_{p}(M)$ we have $L_{\varphi \circ F}^{J}(p ; v)=$ $L_{\varphi}^{J^{\prime}}(F(p), d F(p)(v))$.

(ii) If $z: \mathbb{D} \longrightarrow M$ is a J-holomorphic disc satisfying $z(0)=p$, and $d z(0)\left(e_{1}\right)=v$ (here $e_{1}$ denote the vector $\frac{\partial}{\partial \operatorname{Re}(\zeta)}$ in $\left.\mathbb{R}^{2}\right)$, then $L_{r}^{J}(p ; v)=\Delta(r \circ z)(0)$.

The property (i) expresses the invariance of the Levi form with respect to biholomorphic maps. The property (ii) is often useful in order to compute the Levi form if a vector $t$ is given. 
Proof. (i) Since the map $F$ is $\left(J, J^{\prime}\right)$-holomorphic, we have $J^{\prime *} d r(d F(X))=d r\left(J^{\prime} d F(X)\right)=$ $d r(d F(J X))=d(r \circ F)(J X)$ that is $F^{*}\left(J^{*} d r\right)=J^{*} d(r \circ F)$. By the invariance of the exterior derivative we obtain that $F^{*}\left(d J^{\prime *} d r\right)=d J^{*} d(r \circ F)$. Again using the holomorphy of $F$, we get $d J^{\prime *} d r\left(d F(X), J^{\prime} d F(X)\right)=F^{*}\left(d J^{\prime *} d r\right)(X, J X)=d J^{*} d(r \circ F)(X, J X)$ which implies (i).

(ii) Since $z$ is a $\left(J_{s t}, J\right)$-holomorphic map, (i) implies that $L_{r}^{J}(p, v)=L_{r \circ z}^{J_{s t}}\left(0, e_{1}\right)=\Delta(r \circ$ $z)(0)$. This proves proposition.

It follows from Proposition 1.4.2 that a $\mathcal{C}^{2}$ real valued function $u$ on $M$ is $J$ plurisubharmonic on $M$ if and only if $\mathcal{L}^{J}(u)(p)(v) \geq 0$ for every $p \in M, v \in T_{p} M$.

This leads to the definition :

Definition 1.4.3. $A \mathcal{C}^{2}$ real valued function $u$ on $M$ is strictly $J$-plurisubharmonic on $M$ if $\mathcal{L}^{J}(u)(p)(v)$ is positive for every $p \in M, v \in T_{p} M \backslash\{0\}$.

We have the following example of a $J$-plurisubharmonic function on an almost complex manifold $(M, J)$ :

Example 1.4.4. For every point $p \in(M, J)$ there exists a neighborhood $U$ of $p$ and a diffeomorphism $z: U \rightarrow \mathbb{B}$ centered at $p$ (ie $z(p)=0$ ) such that the function $|z|^{2}$ is $J$ plurisubharmonic on $U$.

We also have the following

Lemma 1.4.5. A function $u$ of class $\mathcal{C}^{2}$ in a neighborhood of a point $p$ of $(M, J)$ is strictly $J$ plurisubharmonic if and only there exists a neighborhood $U$ of $p$ with local complex coordinates $z: U \rightarrow \mathbb{B}$ centered at $p$, such that the function $u-c|z|^{2}$ is J-plurisubharmonic on $U$ for some constant $c>0$.

The function $\log |z|$ is $J_{s t}$-plurisubharmonic on $\mathbb{C}^{n}$ and plays an important role in the pluripotential theory as the Green function for the complex Monge-Ampère operator on the unit ball. In particular, this function is crucially used in Sibony's method in order to localize and estimate the Kobayashi-Royden metric on a complex manifold. Unfortunately, after an arbirarily small general almost complex deformation of the standard structure this function is not plurisubharmonic with respect to the new structure (in any neighborhood of the origin), see for instance [25]. So we will need the following statement communicated to the authors by E.Chirka :

Lemma 1.4.6. Let $p$ be a point in an almost complex manifold $(M, J)$. There exist a neighborhood $U$ of $p$ in $M$, a diffeomorphism $z: U \rightarrow \mathbb{B}$ centered at $p$ and positive constants $\lambda_{0}$, A, such that the function $\log |z|+A|z|$ is $J^{\prime}$-plurisubharmonic on $U$ for every almost complex structure $J^{\prime}$ satisfying $\left\|J^{\prime}-J\right\|_{\mathcal{C}^{2}(\bar{U})} \leq \lambda_{0}$.

Proof. Consider the function $u=|z|$ on $\mathbb{B}$. Since $\mathcal{L}^{J_{s t}}\left(u \circ z^{-1}\right)(w)(v) \geq\|v\|^{2} / 4|w|$ for every $w \in \mathbb{B} \backslash\{0\}$ and every $v \in \mathbb{C}^{n}$, it follows by a direct expansion of $\mathcal{L}^{J^{\prime}}(u)$ that there exist a neighborhood $U$ of $p, U \subset \subset U_{0}$, and a positive constant $\lambda_{0}$ such that $\mathcal{L}^{J^{\prime}}(u)(q)(v) \geq$ $\|v\|^{2} / 5|z(q)|$ for every $q \in U \backslash\{p\}$, every $v \in T_{q} M$ and every almost complex structure $J^{\prime}$ satisfying $\left\|J^{\prime}-J\right\|_{\mathcal{C}^{2}(\bar{U})} \leq \lambda_{0}$. Moreover, computing the Laplacian of $\log |f|$ where $f$ is any $J$-holomorphic disc we obtain, decreasing $\lambda_{0}$ if necessary, that there exists a positive constant $B$ such that $\mathcal{L}^{J^{\prime}}(\log |z|)(q)(v) \geq-B\|v\|^{2} /|z(q)|$ for every $q \in U \backslash\{p\}$, every $v \in T_{q} M$ and 
every almost complex structure $J^{\prime}$ satisfying $\left\|J^{\prime}-J\right\|_{\mathcal{C}^{2}(\bar{U})} \leq \lambda_{0}$. We may choose $A=2 B$ to get the result.

We point out that such constructions of plurisubharmonic functions were generalized recently by J.P.Rosay. He constructed a plurisubharmonic function in $\mathbb{C}^{2}$, whose polar set is a two real dimensional submanifold in $\mathbb{C}^{2}$.

1.4.1. Hopf lemma and the boundary distance preserving property. In what follows we need an analogue to the Hopf lemma for almost complex manifolds. This can be proved quite similarly to the standard one.

Lemma 1.4.7. (Hopf lemma) Let $G$ be a relatively compact domain with a $\mathcal{C}^{2}$ boundary on an almost complex manifold $(M, J)$. Then for any negative $J$-psh function $u$ on $D$ there exists a constant $C>0$ such that $|u(p)| \geq C \operatorname{dist}(p, \partial G)$ for any $p \in G$ (dist is taken with respect to a Riemannian metric on $M$ ).

Proof of Lemma 1.4.7. Step 1. We have the following precise version on the unit disc: let $u$ be a subharmonic function on $\Delta, K$ be a fixed compact on $\Delta$. Suppose that $u<0$ on $\Delta$ and $u \mid K \leq-L$ where $L>0$ is constant. Then there exists $C(K, L)>0$ (independent of $u$ ) such that $|u(p)| \geq \operatorname{Cdist}(p, \partial \Delta)$ (see [66]).

Step 2. Let $G$ be a domain in $\mathbb{C}$ with $\mathcal{C}^{2}$-boundary. Then there exists an $r>0$ (depending on the curvature of the boundary) such that for any boundary point $q \in \partial G$ the ball $B_{q, r}$ of radius $r$ centered on the interior normal to $\partial G$ at $q$, such that $q \in \partial B_{q, r}$, is contained in $G$. Applying Step 1 to the restriction of $u$ on every such a ball (when $q$ runs over $\partial G$ ) we obtain the Hopf lemma for a domain with $\mathcal{C}^{2}$ boundary: let $u$ be a subharmonic function on $G, K$ be a fixed compact on $G$. Suppose that $u<0$ on $G$ and $u \mid K \leq-L$ where $L>0$ is constant. Denote by $k$ the curvature of $\partial G$. Then there exists $C(K, L, k)>0$ (independent of $u$ ) such that $|u(p)| \geq \operatorname{Cdist}(p, \partial \Delta)$.

Step 3. Now we can prove the Hopf lemma for almost complex manifolds. Fix a normal field $v$ on $\partial G$ and consider the family of $J$-holomorphic discs $d_{v}$ satisfying $d_{0}^{\prime}\left(\partial_{x}\right)=v(d(0))$. The image of such a disc is a real surfaces intesecting $\partial G$ transversally, so its pullback gives a $\mathcal{C}^{2}$-curve in $\Delta$. Denote by $G_{v}$ the component of $\Delta$ defined by the condition $d_{v}\left(G_{v}\right) \subset G$. Then every $G_{v}$ is a domain with $\mathcal{C}^{2}$-boundary in $\mathbb{C}$ and the curvatures of boundaries depend continuously on $v$. We conclude by applying Step 2 to the composition $u \circ d_{v}$ on $G_{v}$.

As an application, we obtain the boundary distance preserving property for biholomorphisms between strictly pseudoconvex domains.

Proposition 1.4.8. Let $D$ and $D^{\prime}$ be two smoothly bounded strictly pseudoconvex domains in almost complex manifolds $(M, J)$ and $\left(M^{\prime}, J^{\prime}\right)$ respectively and let $f: D \rightarrow D^{\prime}$ be a $\left(J, J^{\prime}\right)$-biholomorphism. Then there exists a constant $C>0$ such that

$$
(1 / C) \operatorname{dist}\left(f(z), \partial D^{\prime}\right) \leq \operatorname{dist}(z, \partial D) \leq C \operatorname{dist}\left(f(z), \partial D^{\prime}\right) .
$$

Proof of Proposition 1.4.8. According to the previous section, we may assume that $D=\{p$ : $\rho(p)<0\}$ where $\rho$ is a $J$-plurisubharmonic function on $D$, strictly $J$-plurisubharmonic in a neighborhood of the boundary; similarly $D^{\prime}$ can be defined by means of a function $\rho^{\prime}$. Now it suffices to apply the Hopf lemma to the functions $\rho^{\prime} \circ f$ and $\rho \circ f^{-1}$. 
1.4.2. Removable singularities for plurisubharmonic functions. In this subsection we prove the following

Theorem 1.4.9. Let $(M, J)$ be an almost complex manifold of real dimension four and let $E \subset M$ be a generic submanifold of $M$ of real codimension two. Then for any continuous plurisubharmonic function $u$ on $M \backslash E$ the function $u^{*}$ defined by $u^{*}(x)=u(x)$ for $x \in M \backslash E$ and $u^{*}(x)=\lim \sup _{y \in M \backslash E, y \longrightarrow x} u(x)$ for $x \in E$, is J-plurisubharmonic on $M$.

As usual, by a generic manifold we mean a real submanifold $E$ of $(M, J)$ such that the tangent space of $E$ at every point $p \in E$ spans the tangent space $T_{p}(M)$ (considered as a complex space with the structure $J(p))$. In real dimension four, $E$ is generic if and only if it is totally real (for any vector $v \in T_{p}(E) \backslash\{0\}$ the vector $J(p) v$ is not in $T_{p}(E)$ ).

Theorem 1.4.9 deserves some comments.

Firstly, the definition of $u^{*}$ in Theorem 1.4 .9 is correct since $E$ has the empty interior. The function $u^{*}$ is the unique possible plurisubharmonic extension of $u$ on $M$.

Secondly, Theorem 1.4.9 is obtained by N.Karpova [46] in the case where $M$ is a complex manifold. Related results in the integrable case are due to B.Shiffman [71], P.Pflug [64], U.Cegrell [14].

Thirdly, our proof admits easy generalizations to higher dimensions. These are given at the end of this Subsection.

Our method is similar to the approach of [46] and includes two main steps. First, we show that $u$ is upper bounded. The proof uses a filling of $M \backslash E$ by $J$-holomorphic discs. This shows that $u^{*}$ is defined correctly. In order to prove its plurisubharmonicity, we can not use the characterization of upper semi-continuous $J$-plurisubharmonic functions in term of positivity of their Levi current since this result is not yet established for almost complex structures. So we use another approach based on the construction of the plurisubharmonic envelope of an upper semicontinuous function by means of $J$-holomorphic curves; this construction is quite elementary and in the case of the standard structure this is due to Edgar [30] and Bu-Schachermayer [12]; from our point of view, it is of independent interest.

\section{Step 1. Filling by J-holomorphic discs and upper bound for $u^{*}$}

The statement of Theorem 1.4.9 is local. So everywhere below we may assume $M$ is the unit ball $\mathbb{B}_{2} \subset \mathbb{C}^{2}, J=J_{s t}+O(|z|)$ where $z=x+i y$ are standard coordinates in $\mathbb{C}^{2}$ and $J_{s t}$ is the standard complex structure on $\mathbb{C}^{2}$. We also may assume that $E$ coincides with $\mathbb{R}^{2}=\left\{z \in \mathbb{C}^{2}: y=0\right\}$.

Consider the "wedge-type" domains $M_{11}=\left\{y_{1}>0, y_{2}>0\right\}, M_{12}=\left\{y_{1}>0, y_{2}<0\right\}$, $M_{21}=\left\{y_{1}<0, y_{2}>0\right\}, M_{22}=\left\{y_{1}<0, y_{2}<0\right\}$. Then $\mathbb{B}_{2} \backslash \mathbb{R}^{2}=\cup M_{i j}$ so it is enough to show that $u$ is upper bounded on every $M_{i j}$. We prove it for instance on $M_{11}$.

Consider the "support" hypersurface $\Gamma=\{\rho=0\}$ where $\rho=y_{1}+y_{2}+y_{1}^{2}+y_{2}^{2}$ so that $M_{11} \subset \Gamma^{+}=\{\rho>0\}$ and $\bar{M}_{11} \cap \Gamma=\mathbb{R}^{2}$. We may assume that the norm $\left\|J-J_{s t}\right\|_{C^{2}}$ is small enough so that the function $\rho$ is strictly $J$-plurisubharmonic on $\mathbb{B}_{2}$.

For $t \geq 0$ consider the translated hypersurface $\Gamma_{t}=\{z: \rho(z)-t>0\}$ which is strictly $J$-pseudoconvex for small $t$. According to the result of J.F.Barraud-E.Mazzilli [3] and S.Ivashkovich-J.P.Rosay [44] there exists a family $\left(f_{p, t}: \Delta \rightarrow \mathbb{B}_{2}\right.$ of $J$-holomorphic discs with the following properties (see Figure 2) : 
(a) the discs depend smoothly on parameters $p \in \Gamma_{t}, t \geq 0$

(b) there exists an $r>0$ such that for every $p, t$ the surface $f_{p, t}(r \Delta)$ is contained in $\{p\} \cup\{z: \rho(z)-t>0\}$ and $f_{p, t}(0)=p$.

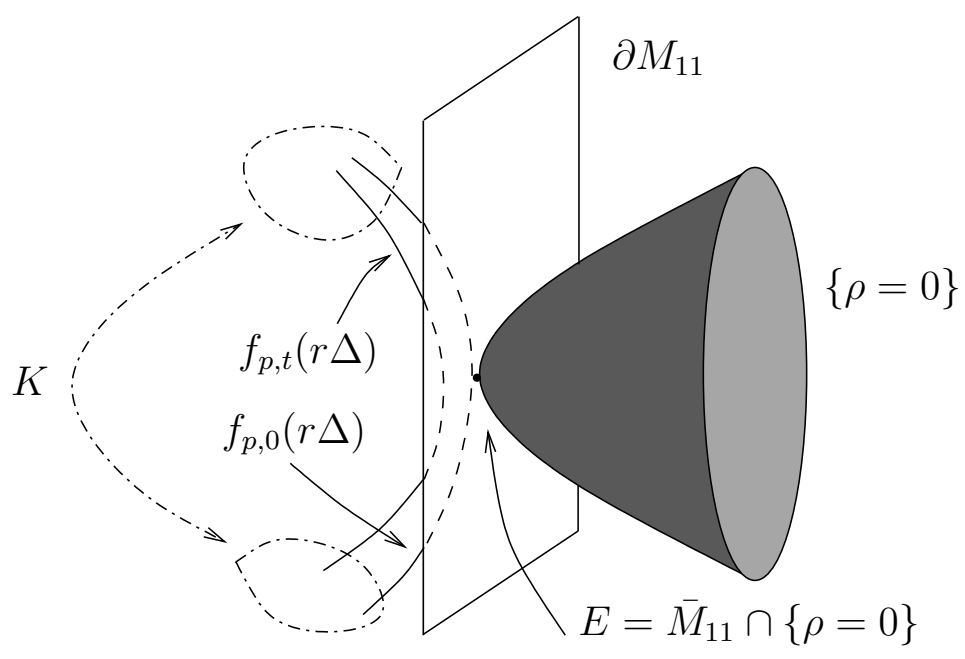

Figure 2

This family of discs fills the "wedge" $M_{11}$ and every disc is contained in $\mathbb{B}_{2} \backslash \mathbb{R}^{2}$. It follows by construction that their boundaries $f(r \partial \Delta)$ form a compact set $K=\cup_{p, t} f(r \partial \Delta)$ in $\mathbb{B}_{2} \backslash \mathbb{R}^{2}$. Since $u$ is bounded above on $K$, say, $u \mid K \leq C$, applying the maximum principle for every subharmonic function $\rho \circ f_{p t}$ we obtain that $u$ is bounded from above by $C$ on $M_{11}$. Repeating this construction for other wedges we obtain the following

Proposition 1.4.10. The function $u$ is upper bounded on $\mathbb{B}_{2} \backslash \mathbb{R}^{2}$.

The standard argument allows now to reduce the proof of Theorem 1.4.9 to the case where $u$ is bounded. Indeed, suppose that Theorem 1.4.9 is proved for bounded functions. For any positive integer $n$, consider the continuous $J$-plurisubharmonic function $u_{n}=\sup (u,-n)$. So $u_{n}^{*}$ is $J$-plurisubharmonic on $\mathbb{B}_{2}$. Since the sequence $\left(u_{n}\right)$ is decreasing, it converges to a $J$-plurisubharmonic function $\hat{u}$ on $\mathbb{B}_{2}$ and $u=\hat{u}$ on $\mathbb{B}_{2} \backslash \mathbb{R}^{2}$. We prove that $u^{*}=\hat{u}$. Clearly, by the uppersemicontinuity of $\hat{u}$ we have $u^{*} \leq \hat{u}$. Fix now a point $x_{0} \in \mathbb{R}^{2}$ and a vector $v \in T_{x_{0}}\left(\mathbb{B}_{2}\right)$ generating a complex line $L$ such that $L \cap T_{x_{0}} \mathbb{R}^{2}=\{0\}$. According to NijenhuisWoolf [61, there exists a $J$-holomorphic disc $f$ such that $f(0)=x_{0}$ and $L$ is tangent to the surface $f(\Delta)$ at $x_{0}$. So the bounded function $u \circ f$ is subharmonic on the punctured disc $\Delta^{*}$ and so extends uniquely as a subharmonic function on $\Delta$ setting $(u \circ f)(0)=\lim \sup _{\zeta \longrightarrow 0} u(\zeta)$. Since this extension is unique, we obtain that $\hat{u}\left(x_{0}\right)=(\hat{u} \circ f)(0)=\lim \sup _{\zeta \longrightarrow 0}(u \circ f)(\zeta) \leq$ $\lim \sup _{z \longrightarrow x_{0}} u(z)=u^{*}\left(x_{0}\right)$. Applying this argument to every point in $\mathbb{R}^{2}$ we obtain that $\hat{u}=u^{*}$ on $\mathbb{B}_{2}$. Thus, everywhere below we assume that $u$ is bounded on $\mathbb{B}_{2} \backslash \mathbb{R}^{2}$.

\section{Step two. $J$-plurisubharmonic envelopes of upper semicontinuous functions}

Denote by $\mu_{r}$ the normalized Lebesgue measure of the disc $r \Delta$ (we simply write $\mu$ if $r=1$ ). 
Proposition 1.4.11. Let $v$ be an upper semicontinuous function on $\mathbb{B}_{2}$. Consider the sequence $\left(v_{n}\right)$ defined as follows: $v_{0}=v$ and for $n \geq 1$, for $z \in \mathbb{B}^{2}$,

$$
v_{n}(z)=\inf \int_{\Delta}\left(v_{n-1} \circ f\right)(\zeta) d \mu,
$$

where inf is taken over all J-holomorphic discs $f: \Delta \rightarrow \mathbb{B}^{2}$ such that $f(0)=z$ (without loss of generality we always assume that every disc $f$ is continuous on $\bar{\Delta}$ and $\left.f(\bar{\Delta}) \subset \mathbb{B}_{2}\right)$. Then the sequence $\left(v_{n}\right)$ decreases pointwise to the largest J-plurisubharmonic function $\hat{v}$ on $\mathbb{B}_{2}$ bounded from above by $v$.

Proof of Proposition 1.4.11. Step 1. The sequence $\left(v_{n}\right)$ decreases. Indeed, for every $z$, the constant disc $f_{z}(\zeta) \equiv z$ is $J$-holomorphic so

$$
v_{n}(z)=\inf _{f} \int_{\Delta}\left(v_{n-1} \circ f\right) d \mu \leq \int_{\Delta} v_{n-1} \circ f_{z} d \mu=v_{n-1}(z) .
$$

Lemma 1.4.12. The function $\hat{v}$ is upper semicontinuous.

Proof of Lemma 1.4.12. We proceed by induction on $n$. For $n=0$ the statement is correct. Suppose that $v_{n-1}$ is upper semicontinuous. Let $\left(z_{k}\right) \subset \mathbb{B}_{2}$ be a sequence of points converging to $z_{0} \in \mathbb{B}_{2}$.

The following claim is a direct consequence of Proposition 1.2.2,

Claim. Let $f: \Delta \rightarrow \mathbb{B}_{2}$ be a J-holomorphic disc continuous on $\bar{\Delta}$ such that $f(0)=z_{0}$ and $f(\bar{\Delta}) \subset \mathbb{B}_{2}$. Then there exists a sequence of J-holomùorphic discs $f_{k}: \Delta \rightarrow \mathbb{B}_{2}$, continuous on $\bar{\Delta}$ such that $f_{k}(\bar{\Delta}) \subset \mathbb{B}_{2}, f_{k}(0)=z_{k}$ for every $k$ and $f_{k} \longrightarrow f$ uniformly on $\Delta$.

Consider the compact set $K$ defined as the closure of the union $\cup_{k} f_{k}(\bar{\Delta})$. Since $v_{n-1}$ is upper semicontinuous it is bounded from above by a constant $C$ on $K$ and

$$
\left(v_{n-1} \circ f\right)(\zeta) \geq \lim \sup _{k \longrightarrow \infty}\left(v_{n-1} \circ f_{k}\right)(\zeta), \zeta \in \Delta .
$$

So by the Fatou lemma

$$
\int_{\Delta}\left(v_{n-1} \circ f d \mu \geq \lim \sup _{k \rightarrow \infty} \int_{\Delta}\left(v_{n-1} \circ f_{k}\right) d \mu \geq \lim \sup _{k \rightarrow \infty} v_{n}\left(z_{k}\right) .\right.
$$

This implies that

$$
v_{n}\left(z_{0}\right)=\inf _{f} \int_{\Delta} v_{n-1} \circ f d \mu \geq \lim \sup _{k \rightarrow \infty} v_{n}\left(z_{k}\right)
$$

and proves Lemma 1.4.12.

Therefore, the function $\hat{v}$ is also upper semicontinuous as a decreasing limit of upper semicontinuous functions.

Step 2. We prove by induction that for any $J$-plurisubharmonic function $\phi$ satisfying $\phi \leq v$ we have $\phi \leq v_{n}$ for any $n$. This is true for $n=0$. Suppose that $\phi \leq v_{n-1}$. Fix an arbitrary point $z_{0} \in \mathbb{B}_{2}$. For every $J$-holomorphic disc $f$ continuous on $\bar{\Delta}$ and satisfying $f(0)=z_{0}, f(\bar{\Delta}) \subset \mathbb{B}_{2}$ we have :

$$
\phi\left(z_{0}\right) \leq \int_{\Delta} \phi \circ f d \mu \leq \int_{\Delta} v_{n-1} \circ f d \mu .
$$

So $v_{n}\left(z_{0}\right) \leq \phi\left(z_{0}\right)$. 
Step 3. We show that the restriction of $\hat{v}$ on a $J$-holomorphic disc is subharmonic. Given $z_{0}$ and $f$ as above, we have

$$
\hat{v}\left(z_{0}\right)=\lim _{n \longrightarrow \infty} v_{n}\left(z_{0}\right) \leq \lim _{n \longrightarrow \infty} \int_{\Delta} v_{n-1} \circ f d \mu=\int_{\Delta} v \circ f d \mu
$$

by the Beppo Levi theorem. This proves Proposition 1.4.11.

Let now $u$ be as in the previous subsection (that is a bounded continuous $J$ plurisubharmonic function on $\mathbb{B}_{2} \backslash \mathbb{R}^{2}$ ) and let $u^{*}$ be its upper semicontinuous extension defined in the statement of Theorem 1.4.9. Setting $v=u^{*}$, we apply Proposition 1.4.11 and obtain the largest $J$-plurisubharmonic function $\hat{v}$ on $\mathbb{B}_{2}$ bounded from above by $v$. In order to conclude the proof of the Theorem 1.4.9, it is sufficient to show that $\hat{v}=u^{*}$ on $\mathbb{B}_{2}$.

The substantial part of the proof is contained in the following

Proposition 1.4.13. For any $z \in \mathbb{B}_{2} \backslash \mathbb{R}^{2}$ we have $\hat{v}(z)=u(z)$.

Proof. Since $\hat{v} \leq u\left(u=u^{*}\right.$ on $\left.\mathbb{B}_{2} \backslash \mathbb{R}^{2}\right)$, we just need to prove the inverse inequality. Using the induction, we show that for every $n, v_{n} \geq u$. For $n=0$ this is clear. Suppose that $v_{n-1} \geq u$ on $\mathbb{B}_{2} \backslash \mathbb{R}^{2}$ (and so $v_{n-1} \geq u^{*}$ on $\mathbb{B}_{2}$ ). Fix a point $z_{0} \in \mathbb{B}_{2} \backslash \mathbb{R}^{2}$ and a $J$-holomorphic disc $f$ satisfying $f(0)=z_{0}$.

Claim. The interior of the set $f^{-1}\left(\mathbb{R}^{2}\right) \subset \Delta$ is empty and $\mu\left(f^{-1}\left(\mathbb{R}^{2}\right)\right)=0$.

Indeed, the set $S \subset \Delta$ of critical points of $f$ is discrete (see [59]). If $p \in f^{-1}\left(\mathbb{R}^{2}\right)$ is not a critical point, the tangent space to $f(\Delta)$ at $f(p)$ is not contained in $T_{f(p)} \mathbb{R}^{2}=\mathbb{R}^{2}$ since $\mathbb{R}^{2}$ is totally real. So in a neighborhood of $p$ the pullback $f^{-1}\left(\mathbb{R}^{2}\right)$ is contained in a smooth real curve. This implies the desired assertion.

Lemma 1.4.14. The function $u \circ f$ defined and subharmonic on $\Delta \backslash f^{-1}\left(\mathbb{R}^{2}\right)$ extends as a subharmonic function on $\Delta$.

Proof. Let $p \in f^{-1}\left(\mathbb{R}^{2}\right)$ be a non-critical point of $f$. Consider a small open disc $U=p+r_{0} \Delta$ centered at $p$. It follows by [61] that

Claim. There exists a sequence of $\left(J_{s t}, J\right)$-holomorphic maps $f_{k}: U \rightarrow \mathbb{B}^{2}$ uniformly converging to $f$ and such that $U \cap f_{k}^{-1}\left(\mathbb{R}^{2}\right)=\{p\}$ for every $k$.

Since $u \circ f_{k}$ is a bounded subharmonic function on $U \backslash\{p\}$, it extends as a subharmonic function on $U$. By continuity of $u$ (this is the only place of the proof where we use this assumption!), the sequence $\left(u \circ f_{k}\right)$ converges to $u \circ f$ on $U \backslash\{p\}$. Therefore, by the Lebesgue dominated convergence theorem, $\left(u \circ f_{k}\right)$ tends to $u \circ f$ on $U$ in the distribution sense and the generalized laplacian $\Delta(u \circ f)$ is a positive distribution. Therefore, $u \circ f$ admits a subharmonic extension $\widetilde{u \circ f}$ on $U$ (given by $\widetilde{u \circ f}(q)=\lim \sup _{\zeta \rightarrow q} u(\zeta)$ for $q \in f^{-1}\left(\mathbb{R}^{2}\right)$ ). Therefore, since the set of critical points of $f$ is discrete, $u \circ f$ extends subharmonically on $\Delta$, proving Lemma 1.4.14.

Using Lemma 1.4.14 and the induction step we have :

$$
\int_{\Delta} v_{n-1} \circ f d \mu \geq \int_{\Delta} u^{*} \circ f d \mu=\int_{\Delta \backslash f^{-1}\left(\mathbb{R}^{2}\right)} u \circ f d \mu \geq u\left(z_{0}\right) .
$$

Hence, $v_{n}\left(z_{0}\right) \geq u\left(z_{0}\right)$ for every $z_{0} \in \mathbb{B}_{2} \backslash \mathbb{R}^{2}$. This proves Proposition 1.4.13. 
Proof of Theorem 1.4.9. Since $\hat{v}$ is upper semicontinuous on $\mathbb{B}_{2}$ and $\hat{v}=u$ on $\mathbb{B}_{2} \backslash \mathbb{R}^{2}$ by Proposition 1.4.13, we have $\hat{v} \geq u^{*}$ on $\mathbb{B}_{2}$. However, $\hat{v} \leq u^{*}$ by construction (see Proposition 1.4.11). Hence $\hat{u}=u^{*}$ on $\mathbb{B}_{2}$. This proves Theorem 1.4.9.

Our method can be easily carried out to almost complex manifolds of any dimension.

Theorem 1.4.15. Let $(M, J)$ be an almost complex manifold and let $E \subset M$ be a genericl submanifold of $M$ of real codimension 2. Then for any continuous plurisubharmonic function $u$ on $M \backslash E$ the function $u^{*}$ defined by $u^{*}(x)=u(x)$ for $x \in M \backslash E$ and $u^{*}(x)=\limsup _{y \in M \backslash E, y \longrightarrow x} u(x)$ for $x \in E$, is J-plurisubharmonic on $M$.

A slight modification of these technics can be used in order to obtain the following almost complex analogues of well-known results. Denote by $\mathcal{H}_{d}$ the Hausdorff measure of dimension $d$.

Theorem 1.4.16. Let $E$ be a subset of an almost complex manifold $(M, J)$ of real dimension $2 n$.

(a) If $\mathcal{H}_{2 n-2}(E) \leq \infty$, then $E$ is removal for every bounded continuous Jplurisubharmonic function.

(b) If $\mathcal{H}_{2 n-3}(E)=0$, then $E$ is removal for every continuous $J$-plurisubharmonic function.

1.5. Canonical almost complex bundles. We present two constructions of almost complex structures on the tangent and on the cotangent bundles of an almost complex manifold.

1.5.1. Lift to the tangent bundle. We endow the tangent bundle $T M$ with the complete lift $J^{c}$ of $J$ (see [82] for its definition). We recall that $J^{c}$ is an almost complex structure on $T M$. Moreover, if $\nabla$ is any $J$ complex connection on $M$ (ie $\nabla J=0$ ) and $\bar{\nabla}$ is the connection defined on $M$ by $\bar{\nabla}_{X} Y=\nabla_{Y} X+[X, Y]$ then $J^{c}$ is the horizontal lift of $J$ with respect to $\bar{\nabla}$. Another definition of $J^{c}$ is given in [57] where this is characterized by a deformation property. The equality between the two definitions given in [82] and in [57] is obtained by their (equal) expression in the local canonical coordinates on $T M$ :

$$
J^{c}=\left(\begin{array}{cc}
J_{i}^{h} & (0) \\
t^{a} \partial_{a} J_{i}^{h} & J_{i}^{h}
\end{array}\right) .
$$

(Here $t^{a}$ are fibers coordinates).

1.5.2. Canonical lift to the cotangent bundle. We recall the definition of the canonical lift of an almost complex structure $J$ on $M$ to the cotangent bundle $T^{*} M$, following [82]. Set $m=2 n$. We use the following notations. Suffixes A,B,C,D take the values 1 to $2 m$, suffixes $a, b, c, \ldots, h, i, j, \ldots$ take the values 1 to $m$ and $\bar{j}=j+m, \ldots$ The summation notation for repeated indices is used. If the notation $\left(\varepsilon_{A B}\right),\left(\varepsilon^{A B}\right),\left(F_{B}^{A}\right)$ is used for matrices, the suffix on the left indicates the column and the suffix on the right indicates the row. We denote local coordinates on $M$ by $\left(x^{1}, \ldots, x^{n}\right)$ and by $\left(p_{1}, \ldots, p_{n}\right)$ the fiber coordinates. 
Recall that the cotangent space $T^{*}(M)$ of $M$ possesses the canonical contact form $\theta$ given in local coordinates by $\theta=p_{i} d x^{i}$. The cotangent lift $\varphi^{*}$ of any diffeomorphism $\varphi$ of $M$ is contact with respect to $\theta$, that is $\theta$ does not depend on the choice of local coordinates on $T^{*}(M)$.

The exterior derivative $d \theta$ of $\theta$ defines the canonical symplectic structure of $T^{*}(M): d \theta=$ $d p_{i} \wedge d x^{i}$ which is also independent of local coordinates in view of the invariance of the exterior derivative. Setting $d \theta=(1 / 2) \varepsilon_{C B} d x^{C} \wedge d x^{B}$ (where $d x^{\bar{j}}=d p_{j}$ ), we have

$$
\left(\varepsilon_{C B}\right)=\left(\begin{array}{cc}
0 & I_{n} \\
-I_{n} & 0
\end{array}\right)
$$

Denote by $\left(\varepsilon^{B A}\right)$ the inverse matrix and write $\varepsilon^{-1}$ for the tensor field of type $(2,0)$ whose component are $\left(\varepsilon^{B A}\right)$. By construction, this definition does not depend on the choice of local coordinates.

Let now $E$ be a tensor field of type $(1,1)$ on $M$. If $E$ has components $E_{i}^{h}$ and $E_{i}^{* h}$ relative to local coordinates $x$ and $x^{*}$ respectively, then $p_{a}^{*} E_{i}^{* a}=p_{a} E_{j}^{b} \frac{\partial x^{j}}{\partial x^{* i}}$. If we interpret a change of coordinates as a diffeomorphism $x^{*}=x^{*}(x)=\varphi(x)$ we denote by $E^{*}$ the direct image of the tensor $E$ under the action of $\varphi$. In the case where $E$ is an almost complex structure (that is $\left.E^{2}=-I d\right)$, then $\varphi$ is a biholomorphism between $(M, E)$ and $\left(M, E^{*}\right)$. Any $(1,1)$ tensor field $E$ on $M$ canonically defines a contact form on $E^{*} M$ via $\sigma=p_{a} E_{b}^{a} d x^{b}$. Since $\left(\varphi^{*}\right)^{*}\left(p_{a}^{*} E_{b}^{* a} d x^{* b}\right)=\sigma, \sigma$ does not depend on a choice of local coordinates (here $\varphi^{*}$ is the cotangent lift of $\varphi$ ). Then this canonically defines the symplectic form

$$
d \sigma=p_{a} \frac{\partial E_{b}^{a}}{\partial x^{c}} d x^{c} \wedge d x^{b}+E_{b}^{a} d p_{a} \wedge d x^{b}
$$

The cotangent lift $\varphi^{*}$ of a diffeomorphism $\varphi$ is a symplectomorphism for $d \sigma$. We may write $d \sigma=(1 / 2) \tau_{C B} d x^{C} \wedge d x^{B}$ where $x^{\bar{i}}=p_{i}$; so we have

$$
\tau_{j i}=p_{a}\left(\frac{\partial E_{i}^{a}}{\partial x^{j}}-\frac{\partial E_{j}^{a}}{\partial x^{i}}\right), \tau_{\bar{j} i}=E_{i}^{j}, \tau_{j \bar{i}}=-E_{j}^{i}, \tau_{\overline{j i}}=0 .
$$

We write $\widehat{E}$ for the tensor field of type $(1,1)$ on $T^{*}(M)$ whose components $\widehat{E}_{B}^{A}$ are given by $\widehat{E}_{B}^{A}=\tau_{B C} \varepsilon^{C A}$. Thus $\widehat{E}_{i}^{h}=E_{i}^{h}, \widehat{E}_{\bar{i}}^{h}=0$ and $\widehat{E}_{i}^{\bar{h}}=p_{a}\left(\frac{\partial E_{i}^{a}}{\partial x^{j}}-\frac{\partial E_{j}^{a}}{\partial x^{i}}\right), \widehat{E}_{\bar{i}}^{\bar{h}}=E_{h}^{i}$. In the matrix form we have

$$
\widehat{E}=\left(\begin{array}{cc}
E_{i}^{h} & 0 \\
p_{a}\left(\frac{\partial E_{i}^{a}}{\partial x^{j}}-\frac{\partial E_{j}^{a}}{\partial x^{i}}\right) & E_{h}^{i}
\end{array}\right) .
$$

By construction, the complete lift $\widehat{E}$ has the following invariance property : if $\varphi$ is a local diffeomorphism of $M$ transforming $E$ to $E^{\prime}$, then the direct image of $\widehat{E}$ under the cotangent lift $\psi:=\varphi^{*}$ is $\widehat{E^{\prime}}$. In general, $\widehat{E}$ is not an almost complex structure, even if $E$ is. Moreover, one can show 82] that $\widehat{J}$ is a complex structure if and only if $J$ is integrable. One may however construct an almost complex structure on $T^{*}(M)$ as follows.

Let $S$ be a tensor field of type $(1, \mathrm{~s})$ on $M$. We may consider the tensor field $\gamma S$ of type $(1, s-1)$ on $T^{*} M$, defined in local canonical coordinates on $T^{*} M$ by the expression 


$$
\gamma S=p_{a} S_{i_{s} \ldots i_{2} i_{1}} d x^{i_{s}} \otimes \cdots \otimes d x^{i_{2}} \otimes \frac{\partial}{\partial p_{i_{1}}} .
$$

In particular, if $T$ is a tensor field of type $(1,2)$ on $M$, then $\gamma T$ has components

$$
\gamma T=\left(\begin{array}{cc}
0 & 0 \\
p_{a} T_{j i}^{a} & 0
\end{array}\right)
$$

in the local canonical coordinates on $T^{*} M$.

Let $F$ be a $(1,1)$ tensor field on $M$. Its Nijenhuis tensor $N$ is the tensor field of type $(1,2)$ on $M$ acting on two vector fields $X$ and $Y$ by

$$
N(X, Y)=[F X, F Y]-F[F X, Y]-F[X, F Y]+F^{2}[X, Y] .
$$

By $N F$ we denote the tensor field acting by $(N F)(X, Y)=N(X, F Y)$. The following proposition is proved in 82 . (p.256).

Proposition 1.5.1. Let $J$ be an almost complex structure on $M$. Then

$$
\tilde{J}:=\widehat{J}+(1 / 2) \gamma(N J)
$$

is an almost complex structure on the cotangent bundle $T^{*}(M)$.

We stress out that the definition of the tensor $\tilde{J}$ is independent of the choice of coordinates on $T^{*} M$. Therefore if $\phi$ is a biholomorphism between two almost complex manifolds $(M, J)$ and $\left(M^{\prime}, J^{\prime}\right)$, then its cotangent lift $\tilde{\phi}:=\left(\phi,{ }^{t} d \phi^{-1}\right)$ is a biholomorphism between $\left(T^{*}(M), \tilde{J}\right)$ and $\left(T^{*}\left(M^{\prime}\right), \tilde{J}^{\prime}\right)$. Indeed one can view $\phi$ as a change of coordinates on $M, J^{\prime}$ representing $J$ in the new coordinates. The cotangent lift $\phi^{*}$ defines a change of coordinates on $T^{*} M$ and $\tilde{J}^{\prime}$ represents $\tilde{J}$ in the new coordinates. So the assertion $(i)$ of Proposition 1.5.1 holds. Property $(i i)$ of Proposition 1.5.1 is immediate in view of the definition of $\tilde{J}$ given by (1.13).

1.5.3. Conormal bundle of a submanifold. The conormal bundle of a strictly pseudoconvex hypersurface in $(M, J)$ provides an important example of a totally real submanifold in the cotangent bundle $T^{*} M$, endowed with the canonical almost complex structure $\tilde{J}$ defined in the last Subsection. If $\Gamma$ is a real submanifold in $M$, the conormal bundle $\Sigma_{J}(\Gamma)$ of $\Gamma$ is the real subbundle of $T_{(1,0)}^{*} M$ defined by

$$
\Sigma_{J}(\Gamma)=\left\{\phi \in T_{(1,0)}^{*} M:\left.\operatorname{Re} \phi\right|_{T \Gamma}=0\right\} .
$$

One can identify the conormal bundle $\Sigma_{J}(\Gamma)$ of $\Gamma$ with any of the following subbundles of $T^{*} M: N_{1}(\Gamma)=\left\{\varphi \in T^{*} M: \varphi_{\mid T \Gamma}=0\right\}$ and $N_{2}(\Gamma)=\left\{\varphi \in T^{*} M: \varphi_{\mid J T \Gamma}=0\right\}$.

Proposition 1.5.2. Let $\Gamma$ be a $\mathcal{C}^{2}$ real hypersurface in $(M, J)$. If $\Gamma$ is strictly $J$ pseudoconvex, then the bundles $N_{1}(\Gamma)$ and $N_{2}(\Gamma)$ (except the zero section) are totally real submanifolds of dimension $2 n$ in $T^{*} M$ equipped with $\tilde{J}$.

Proposition 1.5.2 is due to A.Tumanov [78] in the integrable case. The question whether a similar result was true in the almost complex case was asked by the second author to A.Spiro who gave a positive answer [74]. For completeness we give an alternative proof of this fact. 
Proof of Proposition 1.5.2. Let $x_{0} \in \Gamma$. We consider local coordinates $(x, p)$ for the real cotangent bundle $T^{*} M$ of $M$ in a neighborhood of $x_{0}$. The fiber of $N_{2}(\Gamma)$ is given by $c(x) J^{*} d \rho(x)$, where $c$ is a real nonvanishing function. In what follows we denote $-J^{*} d \rho$ by $d_{J}^{c} \rho$. For every $\varphi \in N_{2}(\Gamma)$ we have $\left.\varphi\right|_{J(T \Gamma)} \equiv 0$. It is equivalenty to prove that $N_{1}(\Gamma)$ is totally real in $\left(T^{*} M, \tilde{J}\right)$ or that $N_{2}(\Gamma)$ is totally real in $\left(T^{*} M, \tilde{J}\right)$. We recall that if $\Theta=p_{i} d x^{i}$ in local coordinates then $d \Theta$ defines the canonical symplectic form on $T^{*} M$. If $V, W \in T\left(N_{2}(\Gamma)\right)$ then $d \Theta(V, W)=0$. Indeed the projection $p r_{1}(V)$ of $V$ (resp. $W$ ) on $M$ is in $J(T \Gamma)$ and the projection of $V$ (resp. $W$ ) on the fiber annihilates $J(T \Gamma)$ by definition. It follows that $N_{2}(\Gamma)$ is a Lagrangian submanifold of $T^{*} M$ for this symplectic form.

Let $V$ be a vector field in $T\left(N_{2}(\Gamma)\right) \cap \tilde{J} T\left(N_{2}(\Gamma)\right)$. We wish to prove that $V=0$. According to what preceeds we have $d \Theta(V, W)=d \Theta(J V, W)=0$ for every $W \in T\left(N_{2}(\Gamma)\right)$. We restrict to $W$ such that $\operatorname{pr}_{1}(W) \in T \Gamma \cap J(T \Gamma)$. Since $\Theta$ is defined over $x_{0} \in \Gamma$ by $\Theta=c d_{J}^{c} \rho$, then $d \Theta=d c \wedge d_{J}^{c} \rho+c d d_{J}^{c} \rho$. Since $d_{J}^{c} \rho\left(p r_{1}(V)\right)=d_{J}^{c} \rho\left(J p r_{1}(V)\right)=d_{J}^{c} \rho\left(p r_{1}(V)\right)=d_{J}^{c} \rho\left(J p r_{1}(V)\right)=$ 0 it follows that $d d_{J}^{c} \rho\left(p r_{1}(V), p r_{1}(\tilde{J} W)\right)=0$. However, by the definition of $\tilde{J}$, we know that $p r_{1}(\tilde{J} W)=J p r_{1}(W)$. Hence, choosing $W=V$, we obtain that $d d_{J}^{c} \rho\left(p r_{1}(V), J p r_{1}(V)\right)=0$. Since $\Gamma$ is strictly $J$-pseudoconvex, it follows that $p r_{1}(V)=0$. In particular, $V$ is given in local coordinates by $V=\left(0, p r_{2}(V)\right)$. It follows now from the form of $\tilde{J}$ that $J V=$ $\left(0, J p r_{2}(V)\right.$ ) (we consider $p r_{2}(V)$ as a vector in $\mathbb{R}^{2 n}$ and $J$ defined on $\mathbb{R}^{2 n}$ ). Since $N_{2}(\Gamma)$ is a real bundle of rank one, then $\operatorname{pr}_{2}(V)$ is equal to zero.

\section{Riemann MAPPing THEOREM FOR ALmost COMPLEX MANifoldS With BOUNDARY}

The aim of this Section is to prove the existence of stationary discs in the ball for small almost complex deformations of the standard structure. Stationary discs are natural global biholomorphic invariants of complex manifolds with boundary. L.Lempert proved in [52] that for a strictly convex domain stationary discs coincide with extremal discs for the Kobayashi metric and studied their basic properties. Using these discs he introduced a multi dimensional analogue of the Riemann map. We define here a local analogue of the Riemann map and establish its main properties. These results were obtained in [23].

\subsection{Existence of discs attached to a real submanifold of an almost complex man- ifold.}

2.1.1. Partial indices and the Riemann-Hilbert problem. In this section we introduce basic tools of the linear Riemann-Hilbert problem.

Let $V \subset \mathbb{C}^{N}$ be an open set. We denote by $\mathcal{C}^{k}(V)$ the Banach space of (real or complex valued) functions of class $\mathcal{C}^{k}$ on $V$ with the standard norm

$$
\|r\|_{k}=\sum_{|\nu| \leq k} \sup \left\{\left|D^{\nu} r(w)\right|: w \in V\right\} .
$$

For a positive real number $\alpha<1$ and a Banach space $X$, we denote by $\mathcal{C}^{\alpha}(\partial \Delta, X)$ the Banach space of all functions $f: \partial \Delta \rightarrow X$ such that

$$
\|f\|_{\alpha}:=\sup _{\zeta \in \partial \Delta}\|f(\zeta)\|+\sup _{\theta, \eta \in \partial \Delta, \theta \neq \eta} \frac{\|f(\theta)-f(\eta)\|}{|\theta-\eta|^{\alpha}}<\infty .
$$


If $\alpha=m+\beta$ with an integer $m \geq 0$ and $\beta \in] 0,1[$, then we consider the Banach space

$$
\mathcal{C}^{\alpha}(V):=\left\{r \in \mathcal{C}^{m}(V, \mathbb{R}): D^{\nu} r \in C^{\beta}(V), \nu,|\nu| \leq m\right\}
$$

and we set $\|r\|_{\alpha}=\sum_{|\nu| \leq m}\left\|D^{\nu} r\right\|_{\beta}$.

Then a map $f$ is in $\mathcal{C}^{\alpha}\left(V, \mathbb{C}^{k}\right)$ if and only if its components belong to $\mathcal{C}^{\alpha}(V)$ and we say that $f$ is of class $\mathcal{C}^{\alpha}$.

Consider the following situation:

- $B$ is an open ball centered at the origin in $\mathbb{C}^{N}$ and $r^{1}, \ldots, r^{N}$ are smooth $\mathcal{C}^{\infty}$ functions defined in a neighborhood of $\partial \Delta \times B$ in $\mathbb{C}^{N} \times \mathbb{C}$

- $f$ is a map of class $\mathcal{C}^{\alpha}$ from $\partial \Delta$ to $B$, where $\alpha>1$ is a noninteger real number

- for every $\zeta \in \partial \Delta$

(i) $E(\zeta)=\left\{z \in B: r^{j}(z, \zeta)=0,1 \leq j \leq N\right\}$ is a maximal totally real submanifold in $\mathbb{C}^{N}$

(ii) $f(\zeta) \in E(\zeta)$,

(iii) $\partial_{z} r^{1}(z, \zeta) \wedge \cdots \wedge \partial_{z} r^{N}(z, \zeta) \neq 0$ on $B \times \partial \Delta$.

Such a family $E=\{E(\zeta)\}$ of manifolds with a fixed disc $f$ is called a totally real fibration over the unit circle. A disc attached to a fixed totally real manifold ( $E$ is independent of $\zeta$ ) is a special case of a totally real fibration.

Assume that the defining function $r:=\left(r^{1}, \ldots, r^{N}\right)$ of $E$ depends smoothly on a small real parameter $\varepsilon$, namely $r=r(z, \zeta, \varepsilon)$, and that the fibration $E_{0}:=E(\zeta, 0)$ corresponding to $\varepsilon=0$ coincides with the above fibration $E$. Then for every sufficiently small $\varepsilon$ and for every $\zeta \in \partial \Delta$ the manifold $E_{\varepsilon}:=E(\zeta, \varepsilon):=\{z \in B: r(z, \zeta, \varepsilon)=0\}$ is totally real. We call $E_{\varepsilon}$ a smooth totally real deformation of the totally real fibration $E$. By a holomorphic disc $\tilde{f}$ attached to $E_{\varepsilon}$ we mean a holomorphic map $\tilde{f}: \Delta \rightarrow B$, continuous on $\bar{\Delta}$, satisfying $r(f(\zeta), \zeta, \varepsilon)=0$ on $\partial \Delta$.

For every positive real noninteger $\alpha$ we denote by $\left(\mathcal{A}^{\alpha}\right)^{N}$ the space of maps defined on $\bar{\Delta}$, $J_{s t}$-holomorphic on $\Delta$, and belonging to $\left(\mathcal{C}^{\alpha}(\bar{\Delta})\right)^{N}$.

2.1.2. Almost complex perturbation of discs. Consider a smooth deformation $\left(J_{\lambda}\right)$ of $J_{s t}$. We recall that for $\lambda$ small enough the $J_{\lambda}$-holomorphicity condition for a map $f: \Delta \rightarrow \mathbb{C}^{N}$ may be written in the form

$$
\bar{\partial}_{J_{\lambda}} f=\bar{\partial} f+q(\lambda, f) \overline{\partial f}=0
$$

where $q$ is a smooth $(n \times n)$ complex matrix satisfying $q(0, \cdot) \equiv 0$.

Let $E_{\varepsilon}=\left\{r_{j}(z, \zeta, \varepsilon)=0,1 \leq j \leq N\right\}$ be a smooth totally real deformation of a totally real fibration $E$. A disc $f \in\left(\mathcal{C}^{\alpha}(\bar{\Delta})\right)^{N}$ is attached to $E_{\varepsilon}$ and is $J_{\lambda}$-holomorphic if and only if it satisfies the following nonlinear boundary Riemann-Hilbert type problem :

$$
\begin{cases}r(f(\zeta), \zeta, \varepsilon) & =0, \quad \zeta \in \partial \Delta \\ \bar{\partial}_{J_{\lambda}} f(\zeta) & =0, \quad \zeta \in \Delta .\end{cases}
$$

Let $f^{0} \in\left(\mathcal{A}^{\alpha}\right)^{N}$ be a disc attached to $E$ and let $\mathcal{U}$ be a neighborhood of $\left(f^{0}, 0,0\right)$ in the space $\left(\mathcal{C}^{\alpha}(\bar{\Delta})\right)^{N} \times \mathbb{R} \times \mathbb{R}$. Given $(f, \varepsilon, \lambda)$ in $U$ define the maps $v_{f, \varepsilon, \lambda}: \zeta \in \partial \Delta \mapsto r(f(\zeta), \zeta, \varepsilon)$ 
and

$$
\begin{aligned}
& u: \mathcal{U} \rightarrow\left(\mathcal{C}^{\alpha}(\partial \Delta)\right)^{N} \times \mathcal{C}^{\alpha-1}(\Delta) \\
& (f, \varepsilon, \lambda) \mapsto\left(v_{f, \varepsilon, \lambda}, \bar{\partial}_{J_{\lambda}} f\right) .
\end{aligned}
$$

Denote by $X$ the Banach space $\left(\mathcal{C}^{\alpha}(\bar{\Delta})\right)^{N}$. Since $r$ is of class $\mathcal{C}^{\infty}$, the map $u$ is smooth and the tangent map $D_{X} u\left(f^{0}, 0,0\right)$ (we consider the derivative with respect to the space $X$ ) is a linear map from $X$ to $\left(\mathcal{C}^{\alpha}(\partial \Delta)\right)^{N} \times \mathcal{C}^{\alpha-1}(\Delta)$, defined for every $h \in X$ by

$$
D_{X} u\left(f^{0}, 0,0\right)(h)=\left(\begin{array}{c}
2 R e[G h] \\
\bar{\partial}_{J_{0}} h
\end{array}\right),
$$

where for $\zeta \in \partial \Delta$

$$
G(\zeta)=\left(\begin{array}{ccc}
\frac{\partial r_{1}}{\partial z^{1}}\left(f^{0}(\zeta), \zeta, 0\right) & \cdots & \frac{\partial r_{1}}{\partial z^{N}}\left(f^{0}(\zeta), \zeta, 0\right) \\
\cdots & \cdots & \cdots \\
\frac{\partial r_{N}}{\partial z^{1}}\left(f^{0}(\zeta), \zeta, 0\right) & \cdots & \frac{\partial r_{N}}{\partial z^{N}}\left(f^{0}(\zeta), \zeta, 0\right)
\end{array}\right)
$$

(see [35]).

Proposition 2.1.1. Let $f^{0}: \bar{\Delta} \rightarrow \mathbb{C}^{N}$ be a $J_{\text {st }}$-holomorphic disc attached to a totally real fibration $E$ in $\mathbb{C}^{N}$. Let $E_{\varepsilon}$ be a smooth totally real deformation of $E$ and $J_{\lambda}$ be a smooth almost complex deformation of $J_{0}$ in a neighborhood of $f(\bar{\Delta})$. Assume that for some $\alpha>1$ the linear map from $\left(\mathcal{A}^{\alpha}\right)^{N}$ to $\left(\mathcal{C}^{\alpha-1}(\Delta)\right)^{N}$ given by $h \mapsto 2 R e[G h]$ is surjective and has a $k$ dimensional kernel. Then there exist $\delta_{0}, \varepsilon_{0}, \lambda_{0}>0$ such that for every $0 \leq \varepsilon \leq \varepsilon_{0}$ and for every $0 \leq \lambda \leq \lambda_{0}$, the set of $J_{\lambda}$-holomorphic discs $f$ attached to $E_{\varepsilon}$ and such that $\left\|f-f^{0}\right\|_{\alpha} \leq \delta_{0}$ forms a smooth $k$-dimensional submanifold $\mathcal{A}_{\varepsilon, \lambda}$ in the Banach space $\left(\mathcal{C}^{\alpha}(\bar{\Delta})\right)^{N}$.

Proof. According to the implicit function Theorem, the proof of Proposition 2.1.1 reduces to the proof of the surjectivity of $D_{X} u$. It follows by classical one-variable results on the resolution of the $\bar{\partial}$-problem in the unit disc that the linear map from $X$ to $\mathcal{C}^{\alpha-1}(\Delta)$ given by $h \mapsto \bar{\partial} h$ is surjective. More precisely, given $g \in \mathcal{C}^{\alpha-1}(\Delta)$ consider the Cauchy transform

$$
T_{C G}(g): \tau \in \Delta \mapsto \frac{1}{2 \pi i} \iint_{\Delta} \frac{g(\zeta)}{\zeta-\tau} d \zeta d \bar{\zeta}
$$

For every function $g \in \mathcal{C}^{\alpha-1}(\Delta)$ the solutions $h \in X$ of the equation $\bar{\partial} h=g$ have the form $h=h_{0}+T_{C G}(g)$ where $h_{0}$ is an arbitrary function in $\left(\mathcal{A}^{\alpha}\right)^{N}$. Consider the equation

$$
D_{X} u\left(f^{0}, 0,0\right)(h)=\left(\begin{array}{l}
g_{1} \\
g_{2}
\end{array}\right)
$$

where $\left(g_{1}, g_{2}\right)$ is a vector-valued function with components $g_{1} \in \mathcal{C}^{\alpha-1}(\partial \Delta)$ and $g_{2} \in \mathcal{C}^{\alpha-1}(\Delta)$. Solving the $\bar{\partial}$-equation for the second component, we reduce (2.2) to

$$
2 \operatorname{Re}\left[G(\zeta) h_{0}(\zeta)\right]=g_{1}-2 \operatorname{Re}\left[G(\zeta) T_{C G}\left(g_{2}\right)(\zeta)\right]
$$

with respect to $h_{0} \in\left(\mathcal{A}^{\alpha}\right)^{N}$. The surjectivity of the map $h_{0} \mapsto 2 R e\left[G h_{0}\right]$ gives the result. 
2.1.3. Riemann-Hilbert problem on the cotangent bundle of an almost complex manifold. Let $\left(J_{\lambda}\right)_{\lambda}$ be an almost complex deformation of the standard structure $J_{s t}$ on $\mathbb{B}_{n}$, satisfying $J_{\lambda}(0)=J_{s t}$. Consider the canonical lift $\tilde{J}_{\lambda}$ on the cotangent bundle, defined by 1.13. In the $(x, y)$ coordinates we identify this with the $(4 n \times 4 n)$-matrix

$$
\tilde{J}_{\lambda}=\left(\begin{array}{cc}
J_{\lambda}(x) & 0 \\
\sum y_{k} A_{\lambda}^{k}(x) & { }^{t} J_{\lambda}(x)
\end{array}\right)
$$

where $A_{\lambda}^{k}(x)=A^{k}(\lambda, x), A_{\lambda}^{k}(x)$ are smooth $(2 n \times 2 n)$-matrix functions. In what follows we always assume that:

$$
A_{0}^{k}(x) \equiv 0, \quad \text { for every } k \text {. }
$$

The trivial bundle $\mathbb{B} \times \mathbb{R}^{2 n}$ over the unit ball is a local coordinate representation of the cotangent bundle of an almost complex manifold. We denote by $x=\left(x^{1}, \ldots, x^{2 n}\right) \in \mathbb{B}_{n}$ and $y=\left(y_{1}, \ldots, y_{2 n}\right) \in \mathbb{R}^{2 n}$ the coordinates on the base and fibers respectively. We identify the base space $\left(\mathbb{R}^{2 n}, x\right)$ with $\left(\mathbb{C}^{n}, z\right)$. Since ${ }^{t} J_{s t}$ is orthogonally equivalent to $J_{s t}$ we may identify $\left(\mathbb{R}^{2 n},{ }^{t} J_{s t}\right)$ with $\left(\mathbb{C}^{n}, J_{s t}\right)$. After this identification the ${ }^{t} J_{s t}$-holomorphicity is expressed by the $\bar{\partial}$-equation in the usual coordinates in $\mathbb{C}^{n}$.

Consider a smooth map $\hat{f}=(f, g): \Delta \rightarrow \mathbb{B} \times \mathbb{R}^{2 n}$ which is $\left(J_{s t}, \tilde{J}_{\lambda}\right)$-holomorphic :

$$
\tilde{J}_{\lambda}(f, g) \circ d \hat{f}=d \hat{f} \circ J_{s t}
$$

on $\Delta$.

For $\lambda$ small enough this can be rewritten as the following Beltrami type quasilinear elliptic equation:

$$
(\mathcal{E})\left\{\begin{array}{cc}
\left.\bar{\partial} f+q_{1}(\lambda, f)\right) \overline{\partial f} & =0 \\
\left.\bar{\partial} g+q_{2}(\lambda, f)\right) \overline{\partial g}+q_{3}(\lambda, f) g+q_{4}(\lambda, f) \bar{g} & =0
\end{array}\right.
$$

where the first equation coincides with the $\left(J_{s t}, J_{\lambda}\right)$-holomorphicity condition for $f$ that is $\left.\bar{\partial}_{J_{\lambda}} f=\bar{\partial} f+q_{1}(\lambda, f)\right) \overline{\partial f}$. The coefficient $q_{1}$ is uniquely determined by $J_{\lambda}$ and, in view of (2.3), the coefficient $q_{k}$ satisfies, for $k=2,3,4$ :

$$
q_{k}(0, \cdot) \equiv 0, q_{k}(\cdot, 0) \equiv 0 .
$$

We point out that in $(\mathcal{E})$ the equations for the fiber component $g$ are obtained as a small perturbation of the standard $\bar{\partial}$-operator. An important feature of this system is that the second equation is linear with respect to the fiber component $g$.

We consider the operator

$$
\bar{\partial}_{\tilde{J}_{\lambda}}:\left(\begin{array}{l}
f \\
g
\end{array}\right) \mapsto\left(\begin{array}{c}
\left.\bar{\partial} f+q_{1}(\lambda, f)\right) \overline{\partial f} \\
\left.\bar{\partial} g+q_{2}(\lambda, f)\right) \overline{\partial g}+q_{3}(\lambda, f) g+q_{4}(\lambda, f) \bar{g}
\end{array}\right) .
$$

Let $r_{j}(z, t, \lambda), j=1, \ldots, 4 n$ be $\mathcal{C}^{\infty}$-smooth real functions on $\mathbb{B} \times \mathbb{B} \times\left[0, \lambda_{0}\right]$ and let $r:=\left(r_{1}, \ldots, r_{4 n}\right)$. Consider the following nonlinear boundary Riemann-Hilbert type problem 
for the operator $\bar{\partial}_{\tilde{J}_{\lambda}}$ :

$$
\left(\mathcal{B} P_{\lambda}\right)\left\{\begin{array}{cc}
r\left(f(\zeta), \zeta^{-1} g(\zeta), \lambda\right) & =0 \text { on } \partial \Delta \\
\bar{\partial}_{\tilde{J}_{\lambda}}(f, g) & =0 \text { on } \Delta
\end{array}\right.
$$

on the space $\mathcal{C}^{\alpha}\left(\bar{\Delta}, \mathbb{B}_{n} \times \mathbb{B}_{n}\right)$.

The boundary problem $\left(\mathcal{B} P_{\lambda}\right)$ has the following geometric meaning. Consider the disc $(\hat{f}, \hat{g}):=\left(f, \zeta^{-1} g\right)$ on $\Delta \backslash\{0\}$ and the set $E_{\lambda}:=\{(z, t): r(z, t, \lambda)=0\}$. The boundary condition in $\left(\mathcal{B} P_{\lambda}\right)$ means that

$$
(\hat{f}, \hat{g})(\partial \Delta) \subset E_{\lambda} .
$$

This boundary problem has the following invariance property. Let $(f, g)$ be a solution of $\left(\mathcal{B} P_{\lambda}\right)$ and let $\phi$ be a automorphism of $\Delta$. Then $(f \circ \phi, c g \circ \phi)$ also satisfies the $\bar{\partial}_{\tilde{J}_{\lambda}}$ equation for every complex constant $c$. In particular, if $\theta \in[0,2 \pi]$ is fixed, then the disc $\left(f\left(e^{i \theta} \zeta\right), e^{-i \theta} g\left(e^{i \theta} \zeta\right)\right)$ satisfies the $\bar{\partial}_{\tilde{J}_{\lambda}}$-equation on $\Delta \backslash\{0\}$ and the boundary of the disc $\left(f\left(e^{i \theta} \zeta\right), e^{-i \theta} \zeta^{-1} g\left(e^{i \theta} \zeta\right)\right)$ is attached to $E_{\lambda}$. This implies the following

Lemma 2.1.2. If $(f, g)$ is a solution of $\left(\mathcal{B} P_{\lambda}\right)$, then $\zeta \mapsto\left(f\left(e^{i \theta} \zeta\right), e^{-i \theta} g\left(e^{i \theta} \zeta\right)\right)$ is also a solution of $\left(\mathcal{B} P_{\lambda}\right)$.

Suppose that this problem has a solution $\left(f^{0}, g^{0}\right)$ for $\lambda=0$ (in view of the above assumptions this solution is holomorphic on $\Delta$ with respect to the standard structure on $\left.\mathbb{C}^{n} \times \mathbb{C}^{n}\right)$. Using the implicit function theorem we study, for sufficiently small $\lambda$, the solutions of $\left(\mathcal{B} P_{\lambda}\right)$ close to $\left(f^{0}, g^{0}\right)$. As above consider the map $u$ defined in a neighborhood of $\left(f^{0}, g^{0}, 0\right)$ in $\left(\mathcal{C}^{\alpha}(\bar{\Delta})\right)^{4 n} \times \mathbb{R}$ by:

$$
u:(f, g, \lambda) \mapsto\left(\begin{array}{c}
\zeta \in \partial \Delta \mapsto r\left(f(\zeta), \zeta^{-1} g(\zeta), \lambda\right) \\
\bar{\partial} f+q_{1}(\lambda, f) \overline{\partial f} \\
\bar{\partial} g+q_{2}(\lambda, f) \overline{\partial g}+q_{3}(\lambda, f) g+q_{4}(\lambda, f) \bar{g}
\end{array}\right) .
$$

If $X:=\left(\mathcal{C}^{\alpha}(\bar{\Delta})\right)^{4 n}$ then its tangent map at $\left(f^{0}, g^{0}, 0\right)$ has the form

$$
D_{X} u\left(f^{0}, g^{0}, 0\right): h=\left(h_{1}, h_{2}\right) \mapsto\left(\begin{array}{c}
\zeta \in \partial \Delta \mapsto 2 R e\left[G\left(f^{0}(\zeta), \zeta^{-1} g^{0}(\zeta), 0\right) h\right] \\
\bar{\partial} h_{1} \\
\bar{\partial} h_{2}
\end{array}\right)
$$

where for $\zeta \in \partial \Delta$ one has

$$
G(\zeta)=\left(\begin{array}{ccc}
\frac{\partial r-1}{\partial w_{1}}\left(f^{0}(\zeta), \zeta^{-1} g^{0}(\zeta), 0\right) & \cdots & \frac{\partial r_{1}}{\partial w_{N}}\left(f^{0}(\zeta), \zeta^{-1} g^{0}(\zeta), 0\right) \\
\cdots & \cdots & \cdots \\
\frac{\partial r_{N}}{\partial w_{1}}\left(f^{0}(\zeta), \zeta^{-1} g^{0}(\zeta), 0\right) & \cdots & \frac{\partial r_{N}}{\partial w_{N}}\left(f^{0}(\zeta), \zeta^{-1} g^{0}(\zeta), 0\right)
\end{array}\right)
$$

with $N=4 n$ and $w=(z, t)$.

If the tangent map $D_{X} u\left(f^{0}, g^{0}, 0\right):\left(\mathcal{A}^{\alpha}\right)^{N} \longrightarrow\left(\mathcal{C}^{\alpha-1}(\Delta)\right)^{N}$ is surjective and has a finite-dimensional kernel, we may apply the implicit function theorem as in Section 2.2 (see Proposition 2.1.1) and conclude to the existence of a finite-dimensional variety of nearby discs. In particular, consider the fibration $E$ over the disc $\left(f^{0}, g^{0}\right)$ with fibers 
$E(\zeta)=\left\{(z, t): r^{j}\left(z, \zeta^{-1} t, 0\right)=0\right\}$. Suppose that this fibration is totally real. Then we have:

Proposition 2.1.3. Suppose that the fibration $E$ is totally real. If the tangent map $D_{X} u\left(f^{0}, g^{0}, 0\right):\left(\mathcal{A}^{\alpha}\right)^{4 n} \longrightarrow\left(\mathcal{C}^{\alpha-1}(\Delta)\right)^{4 n}$ is surjective and has a finite-dimensional kernel, then for every sufficiently small $\lambda$ the solutions of the boundary problem $\left(\mathcal{B} P_{\lambda}\right)$ form a smooth finite dimensional submanifold in the space $\left(\mathcal{C}^{\alpha}(\Delta)\right)^{4 n}$.

In the next Section we present a sufficient condition for the surjectivity of the map $D_{X} u\left(f^{0}, g^{0}, 0\right)$. This is due to J.Globevnik [35, 36] for the integrable case and relies on the partial indices of the totally real fibration along $\left(f^{0}, g^{0}\right)$.

2.2. Generation of stationary discs. Let $D$ be a smoothly bounded domain in $\mathbb{C}^{n}$ with the boundary $\Gamma$. According to [52] a continuous map $f: \bar{\Delta} \rightarrow \bar{D}$, holomorphic on $\Delta$, is called a stationary disc for $D$ (or for $\Gamma$ ) if there exists a holomorphic map $\hat{f}: \Delta \backslash\{0\} \rightarrow T_{(1,0)}^{*}\left(\mathbb{C}^{n}\right)$, $\hat{f} \neq 0$, continuous on $\bar{\Delta} \backslash\{0\}$ and such that

(i) $\pi \circ \hat{f}=f$

(ii) $\zeta \mapsto \zeta \hat{f}(\zeta)$ is in $\mathcal{O}(\Delta)$

(iii) $\hat{f}(\zeta) \in \Sigma_{f(\zeta)}(\Gamma)$ for every $\zeta$ in $\partial \Delta$.

We call $\hat{f}$ a lift of $f$ to the conormal bundle of $\Gamma$ (this is a meromorphic map from $\Delta$ into $T_{(1,0)}^{*}\left(\mathbb{C}^{n}\right)$ whose values on the unit circle lie on $\left.\Sigma(\Gamma)\right)$.

We point out that originally Lempert gave this definition in a different form, using the natural coordinates on the cotangent bundle of $\mathbb{C}^{n}$. The present more geometric version in terms of the conormal bundle is due to Tumanov [78. This form is particularly useful for our goals since it can be transferred to the almost complex case.

Let $f$ be a stationary disc for $\Gamma$. It follows from Proposition 1.5 .2 that if $\Gamma$ is a Levi nondegenerate hypersurface, the conormal bundle $\Sigma(\Gamma)$ is a totally real fibration along $f^{*}$. Conditions $(i)$, (ii), ( iii) may be viewed as a nonlinear boundary problem considered in Section 2. If the associated tangent map is surjective, Proposition 2.1.3 gives a description of all stationary discs $\tilde{f}$ close to $f$, for a small deformation of $\Gamma$. When dealing with the standard complex structure on $\mathbb{C}^{n}$, the bundle $T_{(1,0)}^{*}\left(\mathbb{C}^{n}\right)$ is a holomorphic vector bundle which can be identified, after projectivization of the fibers, with the holomorphic bundle of complex hyperplanes that is with $\mathbb{C}^{n} \times \mathbb{P}^{n-1}$. The conormal bundle $\Sigma(\Gamma)$ of a real hypersurface $\Gamma$ may be naturally identified, after this projectivization, with the bundle of holomorphic tangent spaces $H(\Gamma)$ over $\Gamma$. According to S.Webster [80] this is a totally real submanifold in $\mathbb{C}^{n} \times \mathbb{P}^{n-1}$. When dealing with the standard structure, we may therefore work with projectivizations of lifts of stationary discs attached to the holomorphic tangent bundle $H(\Gamma)$. The technical avantage is that after such a projectivization lifts of stationary discs become holomorphic, since the lifts have at most one pole of order 1 at the origin. This idea was first used by L.Lempert and then applied by several authors [2, 15, 76].

When we consider almost complex deformations of the standard structure (and not just deformations of $\Gamma$ ) the situation is more complicated. If the cotangent bundle $T^{*}\left(\mathbb{R}^{2 n}\right)$ is equipped with $\tilde{J}$, there is no natural possibility to transfer this structure to the space obtained by the projectivization of the fibers. Consequently we do not work with projectivization of 
the cotangent bundle but we will deal with meromorphic lifts of stationary discs. Representing such lifts $(\hat{f}, \hat{g})$ in the form $(\hat{f}, \hat{g})=\left(f, \zeta^{-1} g\right)$, we will consider $\tilde{J}_{\lambda}$-holomorphic discs close to the $J_{s t}$-holomorphic disc $\left(f^{0}, g^{0}\right)$. The disc $(f, g)$ satisfies a nonlinear boundary problem of Riemann-Hilbet type $\left(\mathcal{B} P_{\lambda}\right)$. When an almost complex structure on the cotangent bundle is fixed, we may view it as an elliptic prolongation of an initial almost complex structure on the base and apply the implicit function theorem as in previous section. This avoids difficulties coming from the projectivization of almost complex fibre spaces.

2.2.1. Maslov index and Globevnik's condition. We denote by $G L(N, \mathbb{C})$ the group of invertible $(N \times N)$ complex matrices and by $G L(N, \mathbb{R})$ the group of all such matrices with real entries. Let $0<\alpha<1$ and let $B: \partial \Delta \rightarrow G L\left(N, \mathbb{C}\right.$ ) be of class $\mathcal{C}^{\alpha}$. According to [79] (see also [21]) $B$ admits the factorization $B(\tau)=F^{+}(\tau) \Lambda(\tau) F^{-}(\tau), \tau \in \partial \Delta$, where:

- $\Lambda$ is a diagonal matrix of the form $\Lambda(\tau)=\operatorname{diag}\left(\tau^{k_{1}}, \ldots, \tau^{k_{N}}\right)$,

- $F^{+}: \bar{\Delta} \rightarrow G L(N, \mathbb{C})$ is of class $\mathcal{C}^{\alpha}$ on $\bar{\Delta}$ and holomorphic in $\Delta$,

- $F^{-}:[\mathbb{C} \cup\{\infty\}] \backslash \Delta \rightarrow G L(N, \mathbb{C})$ is of class $\mathcal{C}^{\alpha}$ on $[\mathbb{C} \cup\{\infty\}] \backslash \Delta$ and holomorphic on $[\mathbb{C} \cup\{\infty\}] \backslash \bar{\Delta}$.

The integers $k_{1} \geq \cdots \geq k_{n}$ are called the partial indices of $B$.

Let $E$ be a totally real fibration over the unit circle. For every $\zeta \in \partial \Delta$ consider the "normal" vectors $\nu_{j}(\zeta)=\left(r_{\bar{z}^{1}}^{j}(f(\zeta), \zeta), \ldots, r_{\bar{z}^{N}}^{j}(f(\zeta), \zeta)\right), j=1, \ldots, N$. We denote by $K(\zeta) \in$ $G L(N, \mathbb{C})$ the matrix with rows $\nu_{1}(\zeta), \ldots, \nu_{N}(\zeta)$ and we set $B(\zeta):=-\overline{K(\zeta)}^{-1} K(\zeta), \zeta \in \partial \Delta$. The partial indices of the map $B: \partial \Delta \rightarrow G L(N, \mathbb{C})$ are called the partial indices of the fibration $E$ along the disc $f$ and their sum is called the total index or the Maslov index of $E$ along $f$. The following result is due to J. Globevnik [36]:

Theorem : Suppose that all the partial indices of the totally real fibration $E$ along $f$ are $\geq-1$ and denote by $k$ the Maslov index of $E$ along $f$. Then the linear map from $\left(\mathcal{A}^{\alpha}\right)^{N}$ to $\left(\mathcal{C}^{\alpha-1}(\Delta)\right)^{N}$ given by $h \mapsto 2 R e[G h]$ is surjective and has a $(N+k)$ dimensional kernel.

Proposition 2.1.1 may be restated in terms of partial indices as follows :

Proposition 2.2.1. Let $f^{0}: \bar{\Delta} \rightarrow \mathbb{C}^{N}$ be a $J_{\text {st }}$-holomorphic disc attached to a totally real fibration $E$ in $\mathbb{C}^{N}$. Suppose that all the partial indices of $E$ along $f^{0}$ are $\geq-1$. Denote by $k$ the Maslov index of $E$ along $f^{0}$. Let also $E_{\varepsilon}$ be a smooth totally real deformation of $E$ and $J_{\lambda}$ be a smooth almost complex deformation of $J_{\text {st }}$ in a neighborhood of $f(\bar{\Delta})$. Then there exists $\delta_{0}, \varepsilon_{0}, \lambda_{0}>0$ such that for every $0 \leq \varepsilon \leq \varepsilon_{0}$ and for every $0 \leq \lambda \leq \lambda_{0}$ the set of $J_{\lambda}$-holomorphic discs attached to $E_{\varepsilon}$ and such that $\left\|f-f^{0}\right\|_{\alpha} \leq \delta_{0}$ forms a smooth $(N+k)$-dimensional submanifold $\mathcal{A}_{\varepsilon, \lambda}$ in the Banach space $\left(\mathcal{C}^{\alpha}(\bar{\Delta})\right)^{N}$.

Globevnik's result was applied to the study of stationary discs in some classes of domains in $\mathbb{C}^{n}$ by M.Cerne [15] and A.Spiro-S.Trapani [76]. Since they worked with the projectivization of the conormal bundle, we explicitely compute, for reader's convenience and completeness of exposition, partial indices of meromorphic lifts of stationary discs for the unit sphere in $\mathbb{C}^{n}$. 
Consider the unit sphere $\Gamma:=\left\{z \in \mathbb{C}^{n}: z^{1} \bar{z}^{1}+\cdots+z^{n} \bar{z}^{n}-1=0\right\}$ in $\mathbb{C}^{n}$. The conormal bundle $\Sigma(\Gamma)$ is given in the $(z, t)$ coordinates by the equations

$$
(S)\left\{\begin{array}{c}
z^{1} \bar{z}^{1}+\cdots+z^{n} \bar{z}^{n}-1=0, \\
t_{1}=c \bar{z}^{1}, \ldots, t_{n}=c \bar{z}^{n}, \quad c \in \mathbb{R} .
\end{array}\right.
$$

According to [52, every stationary disc for $\Gamma$ is extremal for the Kobayashi metric. Therefore, such a stationary disc $f^{0}$ centered at the origin is linear by the Schwarz lemma. So, up to a unitary transformation, we have $f^{0}(\zeta)=(\zeta, 0, \ldots, 0)$ with lift $\left(\widehat{f^{0}}, \widehat{g^{0}}\right)(\zeta)=$ $\left(\zeta, 0, \ldots, 0, \zeta^{-1}, 0, \ldots, 0\right)=\left(f^{0}, \zeta^{-1} g^{0}\right)$ to the conormal bundle. Representing nearby meromorphic discs in the form $\left(z, \zeta^{-1} w\right)$ and eliminating the parameter $c$ in system $(S)$ we obtain that holomorphic discs $(z, w)$ close to $\left(f^{0}, g^{0}\right)$ satisfy for $\zeta \in \partial \Delta$ :

$$
\begin{aligned}
r^{1}(z, w)= & z^{1} \bar{z}^{1}+\cdots+z^{n} \bar{z}^{n}-1=0 \\
r^{2}(z, w)= & i z^{1} w_{1} \zeta^{-1}-i \bar{z}^{1} \bar{w}_{1} \zeta=0 \\
r^{3}(z, w)= & \bar{z}^{1} w_{2} \zeta^{-1}-\bar{z}^{2} w_{1} \zeta^{-1}+z^{1} \bar{w}_{2} \zeta-z^{2} \bar{w}_{1} \zeta=0, \\
r^{4}(z, w)= & i \bar{z}^{1} w_{2} \zeta^{-1}-i \bar{z}^{2} w_{1} \zeta^{-1}-i z^{1} \bar{w}_{2} \zeta+i z^{2} \bar{w}_{1} \zeta=0 \\
r^{5}(z, w)= & \bar{z}^{1} w_{3} \zeta^{-1}-\bar{z}^{3} w_{1} \zeta^{-1}+z^{1} \bar{w}_{3} \zeta-z^{3} \bar{w}_{1} \zeta=0, \\
r^{6}(z, w)= & i \bar{z}^{1} w_{3} \zeta^{-1}-i \bar{z}^{3} w_{1} \zeta^{-1}-i z^{1} \bar{w}_{3} \zeta+i z^{3} \bar{w}_{1} \zeta=0, \\
& \cdots \\
r^{2 n-1}(z, w)= & \bar{z}^{1} w_{n} \zeta^{-1}-\bar{z}^{n} w_{1} \zeta^{-1}+z^{1} \bar{w}_{n} \zeta-z^{n} \bar{w}_{1} \zeta=0, \\
r^{2 n}(z, w)= & i \bar{z}^{1} w_{n} \zeta^{-1}-i \bar{z}^{n} w_{1} \zeta^{-1}-i z^{1} \bar{w}_{n} \zeta+i z^{n} \bar{w}_{1} \zeta=0 .
\end{aligned}
$$

Hence the $(2 n \times 2 n)$-matrix $K(\zeta)$ has the following expression:

$$
\left(\begin{array}{cccccccccc}
\zeta & 0 & 0 & \ldots & 0 & 0 & 0 & 0 & \ldots & 0 \\
-i \zeta & 0 & 0 & \ldots & 0 & -i & 0 & 0 & \ldots & 0 \\
0 & -\zeta^{-1} & 0 & \ldots & 0 & 0 & \zeta^{2} & 0 & \ldots & 0 \\
0 & -i \zeta^{-1} & 0 & \ldots & 0 & 0 & -i \zeta^{2} & 0 & \ldots & 0 \\
0 & 0 & -\zeta^{-1} & \ldots & 0 & 0 & 0 & \zeta^{2} & \ldots & 0 \\
0 & 0 & -i \zeta^{-1} & \ldots & 0 & 0 & 0 & -i \zeta^{2} & \ldots & 0 \\
\ldots & \ldots & \ldots & \ldots & \ldots & \ldots & \ldots & \ldots & \ldots & \ldots \\
0 & 0 & 0 & \ldots & -\zeta^{-1} & 0 & 0 & 0 & \ldots & \zeta^{2} \\
0 & 0 & 0 & \ldots & -i \zeta^{-1} & 0 & 0 & 0 & \ldots & -i \zeta^{2}
\end{array}\right)
$$

and a direct computation shows that $-B=\bar{K}^{-1} K$ has the form

$$
\left(\begin{array}{ll}
C_{1} & C_{2} \\
C_{3} & C_{4}
\end{array}\right)
$$

where the $(n \times n)$ matrices $C_{1}, \ldots, C_{4}$ are given by

$$
C_{1}=\left(\begin{array}{cccc}
\zeta^{2} & 0 & . & 0 \\
0 & 0 & . & 0 \\
0 & 0 & . & 0 \\
. & . & . & . \\
0 & 0 & . & 0
\end{array}\right), C_{2}=\left(\begin{array}{cccc}
0 & 0 & . & 0 \\
0 & -\zeta & . & 0 \\
0 & 0 & -\zeta & 0 \\
. & . & . & . \\
0 & 0 & 0 & -\zeta
\end{array}\right)
$$




$$
C_{3}=\left(\begin{array}{cccc}
-2 \zeta & 0 & . & 0 \\
0 & -\zeta & \cdot & 0 \\
0 & 0 & . & 0 \\
. & . & \cdot & \cdot \\
0 & 0 & . & -\zeta
\end{array}\right), C_{4}=\left(\begin{array}{cccc}
-1 & 0 & . & 0 \\
0 & 0 & . & 0 \\
0 & 0 & . & 0 \\
. & . & \cdot & . \\
0 & 0 & . & 0
\end{array}\right)
$$

We point out that the matrix

$$
\left(\begin{array}{ll}
\zeta^{2} & 0 \\
\zeta & 1
\end{array}\right)
$$

admits the following factorization:

$$
\left(\begin{array}{ll}
1 & \zeta \\
0 & 1
\end{array}\right) \times\left(\begin{array}{cc}
-\zeta & 0 \\
0 & \zeta
\end{array}\right) \times\left(\begin{array}{cc}
0 & 1 \\
1 & \zeta^{-1}
\end{array}\right)
$$

Permutating the lines (that is multiplying $B$ by some nondegenerate matrics with constant coefficients) and using the above factorization of $(2 \times 2)$ matrices, we obtain the following

Proposition 2.2.2. All the partial indices of the conormal bundle of the unit sphere along a meromorphic lift of a stationary disc are equal to one and the Maslov index is equal to $2 n$.

Proposition 2.2.2 enables to apply Proposition 2.1.1 to construct the family of stationary discs attached to the unit sphere after a small deformation of the complex structure. Indeed denote by $r_{j}(z, w, \zeta, \lambda) \mathcal{C}^{\infty}$-smooth functions coinciding for $\lambda=0$ with the above functions $r_{1}, \ldots, r_{2 n}$.

In the end of this Subsection we make the two following assumptions:

(i) $r_{1}(z, w, \zeta, \lambda)=z^{1} \bar{z}^{1}+\cdots+z^{n} \bar{z}^{n}-1$, meaning that the sphere is not deformed

(ii) $r_{j}(z, t w, \zeta, \lambda)=\operatorname{tr}^{j}(z, w, \zeta, \lambda)$ for every $j \geq 2, t \in \mathbb{R}$.

Geometrically this means that given $\lambda$, the set $\left\{(z, w): r_{j}(z, w, \lambda)=0\right\}$ is a real vector bundle with one-dimensional fibers over the unit sphere.

Consider an almost complex deformation $J_{\lambda}$ of the standard structure on $\mathbb{B}_{n}$ and its canonical lift $\tilde{J}_{\lambda}$ to the cotangent bundle $\mathbb{B}_{n} \times \mathbb{R}^{2 n}$. Consider now the corresponding boundary problem:

$$
\left(\mathcal{B} P_{\lambda}\right)\left\{\begin{array}{l}
r(f, g, \zeta, \lambda)=0, \zeta \in \partial \Delta, \\
\bar{\partial} f+q_{1}(\lambda, f) \overline{\partial f}=0, \\
\bar{\partial} g+q_{2}(\lambda, f) \overline{\partial g}+q_{3}(\lambda, f) g+q_{4}(\lambda, f) \bar{g}=0 .
\end{array}\right.
$$

Combining Proposition 2.2 .2 with the previous results we obtain the following

Proposition 2.2.3. For every sufficiently small positive $\lambda$, the set of solutions of $\left(\mathcal{B} P_{\lambda}\right)$, close enough to the disc $\left(\widehat{f^{0}}, \widehat{g^{0}}\right)$, forms a smooth $4 n$-dimensional submanifold $V_{\lambda}$ in the space $\mathcal{C}^{\alpha}(\bar{\Delta})$ (for every noninteger $\alpha>1$ ).

Moreover, in view of the assumption (ii) and of the linearity of $(\mathcal{E})$ with respect to the fiber component $g$, we also have the following

Corollary 2.2.4. The projections of discs from $V_{\lambda}$ to the base $\left(\mathbb{R}^{2 n}, J_{\lambda}\right)$ form a $(4 n-1)$ dimensional subvariety in $\mathcal{C}^{\alpha}(\bar{\Delta})$. 
Geometrically the solutions $(f, g)$ of the boundary problem $\left(\mathcal{B} P_{\lambda}\right)$ are such that the discs $\left(f, \zeta^{-1} g\right)$ are attached to the conormal bundle of the unit sphere with respect to the standard structure. In particular, if $\lambda=0$ then every such disc satisfying $f(0)=0$ is linear.

2.3. Canonical Foliation and the "Riemann map" associated with an almost complex structure. In this Section we study the geometry of stationary discs in the unit ball after a small almost complex perturbation of the standard structure. The idea is simple since these discs are small deformations of the complex lines passing through the origin in the unit ball.

2.3.1. Foliation associated with an elliptic prolongation. Fix a vector $v^{0}$ with $\left\|v^{0}\right\|=1$ and consider the corresponding stationary disc $f^{0}: \zeta \mapsto \zeta v^{0}$. Denote by $\left(\widehat{f^{0}}, \widehat{g^{0}}\right)$ its lift to the conormal bundle of the unit sphere. Consider a smooth deformation $J_{\lambda}$ of the standard structure on the unit ball $\mathbb{B}_{n}$ in $\mathbb{C}^{n}$. For sufficiently small $\lambda_{0}$ consider the lift $\tilde{J}_{\lambda}$ on $\mathbb{B}_{n} \times \mathbb{R}^{2 n}$, where $\lambda \leq \lambda_{0}$. Then the solutions of the associated boundary problem $\left(\mathcal{B} P_{\lambda}\right)$ form a $4 n$ parameter family of $\tilde{J}_{\lambda^{-}}$holomorphic maps from $\Delta$ to $\mathbb{C}^{n} \times \mathbb{C}^{n}$. Given such a solution $\left(f^{\lambda}, g^{\lambda}\right)$, consider the disc $\left(\widehat{f^{\lambda}}, \widehat{g^{\lambda}}\right):=\left(f^{\lambda}, \zeta^{-1} g^{\lambda}\right)$. In the case where $\lambda=0$ this is just the lift of a stationary disc for the unit sphere to its conormal bundle. The set of solutions of the problem $\left(\mathcal{B} P_{\lambda}\right)$, close to $\left(\widehat{f^{0}}, \widehat{g^{0}}\right)$, forms a smooth submanifold of real dimension $4 n$ in $\left(\mathcal{C}^{\alpha}(\bar{\Delta})\right)^{4 n}$ according to Proposition 2.2.3. Hence there is a neighborhood $V_{0}$ of $v^{0}$ in $\mathbb{R}^{2 n}$ and a smooth real hypersurface $I_{v^{0}}^{\lambda}$ in $V_{0}$ such that for every $\lambda \leq \lambda_{0}$ and for every $v \in I_{v^{0}}^{\lambda}$ there is one and only one solution $\left(f_{v}^{\lambda}, g_{v}^{\lambda}\right)$ of $\left(\mathcal{B} P_{\lambda}\right)$, up to multiplication of the fiber component $g_{v}^{\lambda}$ by a real constant, such that $f_{v}^{\lambda}(0)=0$ and $d f_{v}^{\lambda}(0)(\partial / \partial \operatorname{Re}(\zeta))=v$.

We may therefore consider the map

$$
F_{0}^{\lambda}:(v, \zeta) \in I_{v^{0}}^{\lambda} \times \bar{\Delta} \mapsto\left(f_{v}^{\lambda}, g_{v}^{\lambda}\right)(\zeta) .
$$

This is a smooth map with respect to $\lambda$ close to the origin in $\mathbb{R}$.

Denote by $\pi$ the canonical projection $\pi: \mathbb{B}_{n} \times \mathbb{R}^{2 n} \rightarrow \mathbb{B}_{n}$ and consider the composition $\widehat{F_{0}^{\lambda}}=\pi \circ F_{0}^{\lambda}$. This is a smooth map defined for $0 \leq \lambda<\lambda_{0}$ and such that

(i) $\widehat{F_{0}^{0}}(v, \zeta)=v \zeta$, for every $\zeta \in \bar{\Delta}$ and for every $v \in I_{v^{0}}^{\lambda}$.

(ii) For every $\lambda \leq \lambda_{0}, \widehat{F_{0}^{\lambda}}(v, 0)=0$.

(iii) For every fixed $\lambda \leq \lambda_{0}$ and every $v \in I_{v^{0}}^{\lambda}$ the map $\widehat{F_{0}^{\lambda}}(v, \cdot)$ is a $J_{\lambda^{-h}}$-holomorphic disc attached to the unit sphere.

(iv) For every fixed $\lambda$, different values of $v \in I_{v^{0}}^{\lambda}$ define different discs.

Definition 2.3.1. We call the family $\left(\widehat{F_{0}^{\lambda}}(v, \cdot)\right)_{v \in I_{v^{0}}^{\lambda}}$ canonical discs associated with the boundary problem $\left(\mathcal{B} P_{\lambda}\right)$.

We stress out that by a canonical disc we always mean a disc centered at the origin. The preceding condition (iv) may be restated as follows:

Lemma 2.3.2. For $\lambda<\lambda_{0}$ every canonical disc is uniquely determined by its tangent vector at the origin. 
In the next Subsection we glue the sets $I_{v}^{\lambda}$, depending on vectors $v \in \mathbb{S}^{2 n-1}$, to define the global indicatrix of $F^{\lambda}$.

2.3.2. Indicatrix. For $\lambda<\lambda_{0}$ consider canonical discs in $\left(\mathbb{B}_{n}, J_{\lambda}\right)$ centered at the origin and admitting lifts close to $\left(\widehat{f^{0}}, \widehat{g^{0}}\right)$.

As above we denote by $I_{v^{0}}^{\lambda}$ the set of tangent vectors at the origin of canonical discs whose lift is close to $\left(\widehat{f^{0}}, \widehat{g^{0}}\right)$. Since these vectors depend smoothly on parameters $v$ close to $v^{0}$ and $\lambda \leq \lambda_{0}, I_{v^{0}}^{\lambda}$ is a smooth deformation of a piece of the unit sphere $\mathbb{S}^{2 n-1}$. So this is a smooth real hypersurface in $\mathbb{C}^{n}$ in a neighborhood of $v^{0}$. Repeating this construction for every vector $v \in \mathbb{S}^{2 n-1}$ we may find a finite covering of $\mathbb{S}^{2 n-1}$ by open connected sets $U_{j}$ such that for every $j$ the nearby stationary discs with tangent vectors at the origin close to $v$ are given by $\widehat{F_{j}^{\lambda}}$. Since every nearby stationary disc is uniquely determined by its tangent vector at the origin, we may glue the maps $\widehat{F_{j}^{\lambda}}$ to the map $\widehat{F^{\lambda}}$ defined for every $v \in \mathbb{S}^{2 n-1}$ and every $\zeta \in \bar{\Delta}$. The tangent vectors of the constructed family of stationary discs form a smooth real hypersurface $I^{\lambda}$ which is a small deformation of the unit sphere. This hypersurface is an analog of the indicatrix for the Kobayashi metric (more precisely, its boundary).

We point out that the local indicatrix $I_{v^{0}}^{\lambda}$ for some fixed $v^{0} \in \mathbb{S}^{2 n-1}$ is also useful.

2.3.3. Circled property and Riemann map. If $\lambda$ is small enough, the hypersurface $I^{\lambda}$ is strictly pseudoconvex with respect to the standard structure. Another important property of the "indicatrix" is its invariance with respect to the linear action of the unit circle.

Let $\lambda \leq \lambda_{0}, v \in I^{\lambda}$ and $f_{v}^{\lambda}:=\widehat{F^{\lambda}}(v, \cdot)$. For $\theta \in \mathbb{R}$ we denote by $f_{v, \theta}^{\lambda}$ the $J_{\lambda^{-}}$-holomorphic disc in $\mathbb{B}_{n}$ defined by $f_{v, \theta}^{\lambda}: \zeta \in \Delta \mapsto f_{v}^{\lambda}\left(e^{i \theta} \zeta\right)$. We have :

Lemma 2.3.3. For every $0 \leq \lambda<\lambda_{0}$, every $v \in I^{\lambda}$ and every $\theta \in \mathbb{R}$ we have $: f_{v, \theta}^{\lambda} \equiv f_{e^{i \theta} v^{\prime}}^{\lambda}$.

Proof. Since $f_{v}^{\lambda}$ is a canonical disc, the disc $f_{v, \theta}^{\lambda}$ has a lift close to the lift of the disc $\zeta \mapsto e^{i \theta} v \zeta$. Then according to Lemma 2.1.2 $f_{v, \theta}^{\lambda}$ is a canonical disc close to the linear disc $\zeta \mapsto e^{i \theta} v \zeta$. Since the first jet of $f_{v, \theta}^{\lambda}$ coincides with the first jet of $f_{e^{i \theta} v}^{\lambda}$, these two nearby stationary discs coincide according to Lemma 2.3.2.

This statement implies that for any $w \in I^{\lambda}$ the vector $e^{i \theta} w$ is in $I^{\lambda}$ as well.

It follows from the above arguments that there exists a natural parametrization of the set of canonical discs by their tangent vectors at the origin, that is by the points of $I^{\lambda}$. The map

$$
\begin{aligned}
\widehat{F^{\lambda}}: I^{\lambda} \times \Delta & \rightarrow \mathbb{B}_{n} \\
(v, \zeta) & \mapsto f_{v}^{\lambda}(\zeta)
\end{aligned}
$$

is smooth on $I^{\lambda} \times \Delta$. Moreover, if we fix a small positive constant $\varepsilon_{0}$, then by shrinking $\lambda_{0}$ if necessary there is, for every $\lambda<\lambda_{0}$, a smooth function $\widehat{G^{\lambda}}$ defined on $I^{\lambda} \times \Delta$, satisfying $\left\|\widehat{G^{\lambda}(v, \zeta)}\right\| \leq \varepsilon_{0}|\zeta|^{2}$ on $I^{\lambda} \times \Delta$, such that for every $\lambda<\lambda_{0}$ we have on $I^{\lambda} \times \Delta$ :

$$
\widehat{F^{\lambda}}(v, \zeta)=\zeta v+\widehat{G^{\lambda}}(v, \zeta)
$$


Consider now the restriction of $\widehat{F^{\lambda}}$ to $I^{\lambda} \times[0,1]$. This is a smooth map, still denoted by $\widehat{F^{\lambda}}$. We have the following :

Proposition 2.3.4. There exists $\lambda_{1} \leq \lambda_{0}$ such that for every $\lambda<\lambda_{1}$ the family $\left(\widehat{F^{\lambda}}(v, r)\right)_{(v, r) \in I^{\lambda} \times[0,1[}$ is a real foliation of $\mathbb{B}_{n} \backslash\{0\}$.

Proof. Step 1. For $r \neq 0$ we write $w:=r v$. Then $r=\|w\|, v=w /\|w\|$ and we denote by $\widetilde{F^{\lambda}}$ the function $\widetilde{F^{\lambda}}(w):=\widehat{F^{\lambda}}(v, r)$. For $\lambda<\lambda_{0}, \widetilde{F^{\lambda}}$ is a smooth map of the variable $w$ on $\mathbb{B}_{n} \backslash\{0\}$, satisfying :

$$
\widetilde{F^{\lambda}}(w)=w+\widetilde{G^{\lambda}}(w)
$$

where $\widetilde{G^{\lambda}}$ is a smooth map on $\mathbb{B}_{n} \backslash\{0\}$ with $\left\|\tilde{G}^{\lambda}(w)\right\| \leq \varepsilon_{0}\|w\|^{2}$ on $\mathbb{B}_{n} \backslash\{0\}$. This implies that $\widetilde{F^{\lambda}}$ is a local diffeomorphism at each point in $\mathbb{B}_{n} \backslash\{0\}$, and so that $\widehat{F^{\lambda}}$ is a local diffeomorphism at each point in $\left.I^{\lambda} \times\right] 0,1\left[\right.$. Moreover, the condition $\left\|\widetilde{G^{\lambda}}(w)\right\| \leq \varepsilon_{1}\|w\|^{2}$ on $\mathbb{B}_{n} \backslash\{0\}$ implies that $\widetilde{G^{\lambda}}$ is differentiable at the origin with $d \widetilde{G^{\lambda}}(0)=0$. Hence by the implicit function theorem there exists $\lambda_{1}<\lambda_{0}$ such that the map $\widetilde{F^{\lambda}}$ is a local diffeomorphism at the origin for $\lambda<\lambda_{1}$. So there exists $0<r_{1}<1$ and a neighborhood $U$ of the origin in $\mathbb{C}^{n}$ such that $\widehat{F^{\lambda}}$ is a diffeomorphism from $\left.I^{\lambda} \times\right] 0, r_{1}\left[\right.$ to $U \backslash\{0\}$, for $\lambda<\lambda_{1}$.

Step 2. We show that $\widehat{F^{\lambda}}$ is injective on $\left.\left.I^{\lambda} \times\right] 0,1\right]$ for sufficiently small $\lambda$. Assume by contradiction that for every $n$ there exist $\left.\left.\lambda_{n} \in \mathbb{R}, r_{n}, r_{n}^{\prime} \in\right] 0,1\right], v^{n}, w^{n} \in I^{\lambda_{n}}$ such that:

- $\lim _{n \rightarrow \infty} \lambda_{n}=0, \lim _{n \rightarrow \infty} r_{n}=r, \lim _{n \rightarrow \infty} r_{n}^{\prime}=r^{\prime}$,

- $\lim _{n \rightarrow \infty} v^{n}=v \in \mathbb{S}^{2 n-1}, \lim _{n \rightarrow \infty} w^{n}=w \in \mathbb{S}^{2 n-1}$

and satisfying

$$
\widehat{F^{\lambda_{n}}}\left(v^{n}, r_{n}\right)=\widehat{F^{\lambda_{n}}}\left(w^{n}, r_{n}^{\prime}\right)
$$

for every $n$. Since $\widehat{F}$ is smooth with respect to $\lambda, v, r$, it follows that $\widehat{F^{0}}(v, r)=\widehat{F^{0}}\left(w, r^{\prime}\right)$ and so $v=w$ and $r=r^{\prime}$. If $r<r_{1}$ then the contradiction follows from the fact that $\widehat{F^{\lambda}}$ is a diffeomorphism from $\left.I^{\lambda} \times\right] 0, r_{1}$ [ to $U \backslash\{0\}$. If $r \geq r_{1}$ then for every neighborhood $U_{\infty}$ of $r v$ in $\mathbb{B}_{n} \backslash\{0\}, r_{n} v^{n} \in U_{\infty}$ and $r_{n}^{\prime} w^{n} \in U_{\infty}$ for sufficiently large $n$. Since we may choose $U_{\infty}$ such that $\widehat{F^{\lambda}}$ is a diffeomorphism from a neighborhood of $(v, r)$ in $\left.\left.I^{\lambda} \times\right] r_{1}, 1\right]$ uniformly with respect to $\lambda<<1$, we still obtain a contradiction.

Step 3. We show that $\widehat{F^{\lambda}}$ is surjective from $\left.I^{\lambda} \times\right] 0,1\left[\right.$ to $\mathbb{B}_{n} \backslash\{0\}$. It is sufficient to show that $\widehat{F^{\lambda}}$ is surjective from $I^{\lambda} \times\left[r_{1}, 1\left[\right.\right.$ to $\mathbb{B}_{n} \backslash U$. Consider the nonempty set $E_{\lambda}=\left\{w \in \mathbb{B}_{n} \backslash U: w=\right.$ $\widehat{F^{\lambda}}(v, r)$ for some $\left.(v, r) \in I^{\lambda} \times\right] r_{1}, 1[\}$. Since the jacobian of $\widehat{F^{\lambda}}$ does not vanish for $\lambda=0$ and $\widehat{F^{\lambda}}$ is smooth with respect to $\lambda$, the set $E_{\lambda}$ is open for sufficiently small $\lambda$. Moreover it follows immediately from its definition that $E_{\lambda}$ is also closed in $\mathbb{B}_{n} \backslash U$. Thus $E_{\lambda}=\mathbb{B}_{n} \backslash U$.

These three steps prove the result.

We can construct now the map $\Psi_{J_{\lambda}}$ for $\lambda<\lambda_{1}$. For every $z \in \mathbb{B}_{n} \backslash\{0\}$ consider the unique couple $\left.(v(z), r(z)) \in I^{\lambda} \times\right] 0,1$ [ such that $f_{v}^{\lambda}$ is the unique canonical disc passing through $z$ (its existence and unicity are given by Proposition 2.3.4) with $f_{v(z)}^{\lambda}(0)=0, d f_{v(z)}^{\lambda}(0)(\partial / \partial R e(\zeta))=$ 
$v(z)$ and $f_{v(z)}^{\lambda}(r(z))=z$. The map $\Psi_{J_{\lambda}}$ is defined by :

$$
\begin{aligned}
\Psi_{J_{\lambda}}: \overline{\mathbb{B}}_{n} \backslash\{0\} & \rightarrow \mathbb{C}^{n} \\
z & \mapsto r(z) v(z) .
\end{aligned}
$$

Definition 2.3.5. The map $\Psi_{J_{\lambda}}$ is called the Riemann map associated with the almost complex structure $J_{\lambda}$.

This map is an analogue of the circular representation of a strictly convex domain introduced by L.Lempert [52]. The term "Riemann map" was used by S. Semmes [70] for a slightly different map where the vector $v(z)$ is normalized (and so such a map takes values in the unit ball). In this paper we work with the indicatrix since this is more convenient for our applications.

The Riemann map $\Psi_{J_{\lambda}}$ has the following properties :

Proposition 2.3.6. (i) For every $(v, \zeta) \in I^{\lambda} \times \Delta$ we have $\left(\Psi_{J_{\lambda}} \circ f_{v}^{\lambda}\right)(\zeta)=\zeta v$ and so $\log \left\|\left(\Psi_{J_{\lambda}} \circ f_{v}^{\lambda}\right)(\zeta)\right\|=\log |\zeta|$.

(ii) There exist constants $0<C^{\prime}<C$ such that $C^{\prime}\|z\| \leq\left\|\Psi_{J_{\lambda}}(z)\right\| \leq C\|z\|$ on $\mathbb{B}_{n}$.

Proof. (i) Let $\zeta=e^{i \theta} r \in \Delta\left(0, r_{0}\right)$ with $\theta \in\left[0,2 \pi\left[\right.\right.$. Then $f_{v}^{\lambda}(\zeta)=f_{v}^{\lambda}\left(e^{i \theta} r\right)=f_{e^{i \theta} v}^{\lambda}(r)$. Hence we have $\left(\Psi_{J_{\lambda}} \circ f_{v}^{\lambda}\right)(\zeta)=\Psi_{J_{\lambda}}\left(f_{e^{i \theta} v}^{\lambda}(r)\right)=e^{i \theta} v r=\zeta v$.

(ii) Let $z \in \mathbb{B}_{n} \backslash\{0\}$. Then according to equation (2.5) we have the inequality $\left\|\Psi_{J_{\lambda}}(z)\right\|(1-$ $\left.\varepsilon_{1}\left\|\Psi_{J_{\lambda}}(z)\right\|\right) \leq\|z\|^{2} \leq\left\|\Psi_{J_{\lambda}}(z)\right\|\left(1+\varepsilon_{1}\left\|\Psi_{J_{\lambda}}(z)\right\|\right)$. Since $\left\|\Psi_{J_{\lambda}}(z)\right\| \leq 1$ we obtain the desired inequality with $c^{\prime}=1 / 1+\varepsilon_{1}$ and $c=1 / 1-\varepsilon_{1}$.

From the above analysis we deduce the following basic properties of the Riemann map.

Proposition 2.3.7.

(i) The indicatrix $I^{\lambda}$ is a compact circled smooth $J_{\lambda}$-strictly pseudoconvex hypersurface bounding a domain denoted by $\Omega^{\lambda}$.

(ii) The Riemann map $\Psi_{J_{\lambda}}: \overline{\mathbb{B}}_{n} \backslash\{0\} \rightarrow \bar{\Omega}^{\lambda} \backslash\{0\}$ is a smooth diffeomorphism.

(iii) For every canonical disc $f_{v}^{\lambda}$ we have $\Psi_{J_{\lambda}} \circ f_{v}^{\lambda}(\zeta)=v \zeta$.

2.3.4. Local Riemann map. We introduce the notion of local indicatrix $I_{v^{0}}^{\lambda}$ for $v^{0} \in \mathbb{S}^{2 n-1}$. We may localize the notion of the Riemann map, introducing a similar associated with the local indicatrix. Denote by $\Omega_{v^{0}}^{\lambda}$ the set $I_{v^{0}}^{\lambda} \times[0,1[$. The arguments used in the proof of Proposition 2.3.4 show that $\widehat{F^{\lambda}}\left(\Omega_{v^{0}}^{\lambda}\right)$ is foliated by stationary discs centered at the origin. We may therefore define the Riemann map $\Psi_{J_{\lambda}, v^{0}}$ on $\widehat{F^{\lambda}}\left(\Omega_{v^{0}}^{\lambda}\right)$ by:

$$
\Psi_{J_{\lambda}, v^{0}}(z)=r(z) v(z)
$$

where $v(z)$ is the tangent vector at the origin of the unique stationary disc $f_{v(z)}^{\lambda}$ passing through $z$ and $f_{v(z)}^{\lambda}(r(z))=z$.

Remark 2.3.8. We point out that the Riemann map can be defined in any sufficiently small deformation of the unit ball and satisfies all the same properties. Moreover one can easily generalize this construction to strictly convex domains in $\mathbb{C}^{n}$ equipped with small almost complex deformations of the standard structure. 
2.3.5. Structure properties of the Riemann map. Assume now that $M \subset \mathbb{C}^{n}$ and let $J_{\lambda}$ be an almost complex deformation of the standard structure on $M$. Let $i: T_{(1,0)}^{*}(M, J) \rightarrow$ $T^{*}(M)$ be the canonical identification. Let $D$ be a smoothly bounded domain in $M$ with the boundary $\Gamma$. The conormal bundle $\Sigma_{J}(\Gamma)$ of $\Gamma$ is a real subbundle of $\left.T_{(1,0)}^{*}(M, J)\right|_{\Gamma}$ whose fiber at $z \in \Gamma$ is defined by $\Sigma_{z}(\Gamma)=\left\{\phi \in T_{(1,0)}^{*}(M, J): \operatorname{Re} \phi \mid H_{(1,0)}^{J}(\Gamma)=0\right\}$. Since the form $\partial_{J} \rho$ forms a basis in $\Sigma_{J}(\Gamma)$, every $\phi \in \Sigma_{J}(\Gamma)$ has the form $\phi=c \partial_{J} \rho, c \in \mathbb{R}$.

Definition 2.3.9. A continuous map $f: \bar{\Delta} \rightarrow(\bar{D}, J)$, J-holomorphic on $\Delta$, is called a stationary disc if there exists a smooth map $\hat{f}=(f, g): \Delta \backslash\{0\} \rightarrow T_{(1,0)}^{*}(M, J), \hat{f} \neq 0$ which is continuous on $\bar{\Delta} \backslash\{0\}$ and such that

(i) $\zeta \mapsto \hat{f}(\zeta)$ satisfies the $\bar{\partial}_{\tilde{J}_{\lambda}}$-equation on $\Delta \backslash\{0\}$,

(ii) $\left(i \circ\left(f, \zeta^{-1} g\right)\right)(\partial \Delta) \subset \Sigma_{J}(\Gamma)$.

We call $\hat{f}$ a lift of $f$ to the conormal bundle of $\Gamma$. Clearly the notion of a stationary disc is invariant in the following sense: if $\phi$ is a $\mathcal{C}^{1}$ diffeomorphism between $\bar{D}$ and $\bar{D}^{\prime}$ and a $\left(J, J^{\prime}\right)$ biholomorphism from $D$ to $D^{\prime}$, then for every stationary disc $f$ in $(D, J)$ the composition $\phi \circ f$ is a stationary discs in $\left(D^{\prime}, J^{\prime}\right)$.

Let now $D$ coincide with the unit ball $\mathbb{B}_{n}$ equipped with an almost complex deformation $J_{\lambda}$ of the standard structure. Then it follows by definition that stationary discs in $\left(\mathbb{B}_{n}, J_{\lambda}\right)$ may be described as solutions of a nonlinear boundary problem $\left(\mathcal{B} P_{\lambda}\right)$ associated with $J_{\lambda}$. The above techniques give the existence and efficient parametrization of the variety of stationary discs in $\left(\mathbb{B}_{n}, J_{\lambda}\right)$ for $\lambda$ small enough. This allows to apply the definition of the Riemann map and gives its existence. We sum up our considerations in the following Theorem, giving the main structural properties of the Riemann map.

Theorem 2.3.10. Let $J_{\lambda}, J_{\lambda}^{\prime}$ be almost complex perturbations of the standard structure on $\overline{\mathbb{B}}_{n}$. The Riemann map $\Psi_{J_{\lambda}}$ exists for sufficiently small $\lambda$ and satisfies the following properties:

(a) $\Psi_{J_{\lambda}}: \overline{\mathbb{B}}_{n} \backslash\{0\} \rightarrow \bar{\Omega}^{\lambda} \backslash\{0\}$ is a smooth diffeomorphism

(b) The restriction of $\Psi_{J_{\lambda}}$ on every stationary disc through the origin is $\left(J_{s t}, J_{s t}\right)$ holomorphic (and even linear)

(c) $\Psi_{J_{\lambda}}$ commutes with biholomorphisms. More precisely for sufficiently small $\lambda^{\prime}$ and for every $\mathcal{C}^{1}$ diffeomorphism $\varphi$ of $\overline{\mathbb{B}}_{n},\left(J_{\lambda}, J_{\lambda^{\prime}}\right)$-holomorphic in $\mathbb{B}_{n}$ and satisfying $\varphi(0)=0$, we have

$$
\varphi=\left(\Psi_{J_{\lambda^{\prime}}}\right)^{-1} \circ d \varphi_{0} \circ \Psi_{J_{\lambda}} .
$$

Proof of Theorem 2.3.10. Conditions (a) and (b) are conditions (i) and (ii) of Proposition 2.3.7.

For condition $(\mathrm{c})$, let $\varphi:\left(\mathbb{B}_{n}, J\right) \rightarrow\left(\mathbb{B}_{n}, J^{\prime}\right)$ be a $\left(J, J^{\prime}\right)$-biholomorphism of class $\mathcal{C}^{1}$ on $\overline{\mathbb{B}}_{n}$ satisfying $\varphi(0)=0$. We know that a disc $f_{v}^{J}$ is a canonical disc for the almost complex structure $J$ if and only if $\varphi\left(f_{v}^{J}\right)$ is a canonical disc for the almost complex structure $J^{\prime}$. Since $\varphi\left(f_{v}^{J}\right)=f_{d \varphi_{0}(v)}^{J^{\prime}}$ by definition, $\Psi_{J}\left(f_{v}^{J}\right)(\zeta)=\zeta v$ and $\Psi_{J^{\prime}}\left(f_{d \varphi_{0}(v)}\right)(\zeta)=\zeta d \varphi_{0}(v)$ by Proposition 2.3.7(iii), condition (c) follows from the following diagram (see Figure 3), which ends the proof of Theorem 2.3.10: 


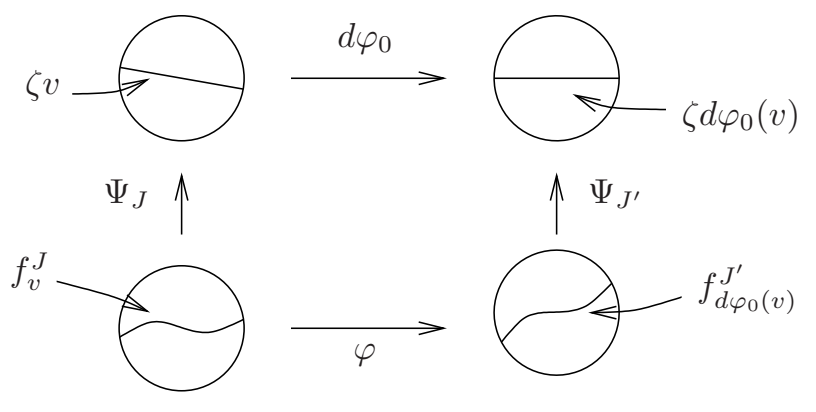

Figure 3

Riemann maps are useful for the boundary study of biholomorphisms in almost complex manifolds. We have

Corollary 2.3.11. If $\lambda, \lambda^{\prime}<<1$ and $\varphi$ is a $\mathcal{C}^{1}$ diffeomorphism of $\overline{\mathbb{B}}_{n}$, $\left(J_{\lambda}, J_{\lambda^{\prime}}\right)$-holomorphic in $\mathbb{B}_{n}$ satisfying $\varphi(0)=0$, then $\varphi$ is of class $\mathcal{C}^{\infty}$ on $\overline{\mathbb{B}}_{n}$.

Proof. This follows immediately by Theorem 2.3.10 condition (c) since the Riemann map is smooth up to the boundary.

2.3.6. Rigidity and local equivalence problem. Condition (c) of Theorem 2.3.10 implies the following partial generalization of Cartan's theorem for almost complex manifolds:

Corollary 2.3.12. If $\lambda<<1$ and if $\varphi$ is a $\mathcal{C}^{1}$ diffeomorphism of $\overline{\mathbb{B}}_{n},\left(J_{\lambda}, J_{\lambda}\right)$-holomorphic in $\mathbb{B}_{n}$, satisfying $\varphi(0)=0$ and $d \varphi(0)=I$ then $\varphi$ is the identity.

This provides an efficient parametrization of the isotropy group of the group of biholomorphisms of $\left(\mathbb{B}_{n}, J_{\lambda}\right)$.

We can solve the local biholomorphic equivalence problem between almost complex manifolds in terms of the Riemann map similarly to [11, 55] (see the paper [58] by P. Libermann for a traditional approach to this problem based on Cartan's equivalence method for $G$ structures). Let $I^{\lambda}$ (resp. $\left.\left(I^{\prime}\right)^{\lambda}\right)$ be the indicatrix of $\left(\mathbb{B}_{n}, J_{\lambda}\right)$ (resp. $\left.\left(\mathbb{B}_{n}, J_{\lambda}^{\prime}\right)\right)$ bounding the domain $\Omega^{\lambda}$ (resp. $\left.\left(\Omega^{\prime}\right)^{\lambda}\right)$ and let $\Psi_{J_{\lambda}}$ (resp. $\Psi_{J_{\lambda}^{\prime}}$ ) be the associated Riemann map. This induces the almost complex structure $J_{\lambda}^{*}:=d \Psi_{J_{\lambda}} \circ J_{\lambda} \circ d\left(\Psi_{J_{\lambda}}\right)^{-1}\left(\operatorname{resp} .\left(J_{\lambda}^{\prime}\right)^{*}:=d \Psi_{J_{\lambda}^{\prime}} \circ J_{\lambda} \circ d\left(\Psi_{J_{\lambda}^{\prime}}\right)^{-1}\right)$ on $\Omega^{\lambda}$ (resp. $\left.\left(\Omega^{\prime}\right)^{\lambda}\right)$. Then we have:

Theorem 2.3.13. The following conditions are equivalent:

(i) There exists a $\mathcal{C}^{\infty}$ diffeomorphism $\varphi$ of $\overline{\mathbb{B}}_{n},\left(J_{\lambda}, J_{\lambda}^{\prime}\right)$-holomorphic on $\mathbb{B}_{n}$ and satisfying $\varphi(0)=0$,

(ii) There exists a $J_{\text {st }}$-linear isomorphism $L$ of $\mathbb{C}^{n},\left(J_{\lambda}^{*},\left(J_{\lambda}^{\prime}\right)^{*}\right)$-holomorphic on $\Omega^{\lambda}$ and such that $L\left(\Omega^{\lambda}\right)=\left(\Omega^{\prime}\right)^{\lambda}$.

Proof. If $\varphi$ satisfies condition $(i)$, then $L:=d \varphi_{0}$ satisfies condition $(i i)$, in view of the commutativity of the following diagram (see Figure 4) given by Theorem 2.3 .10 : 


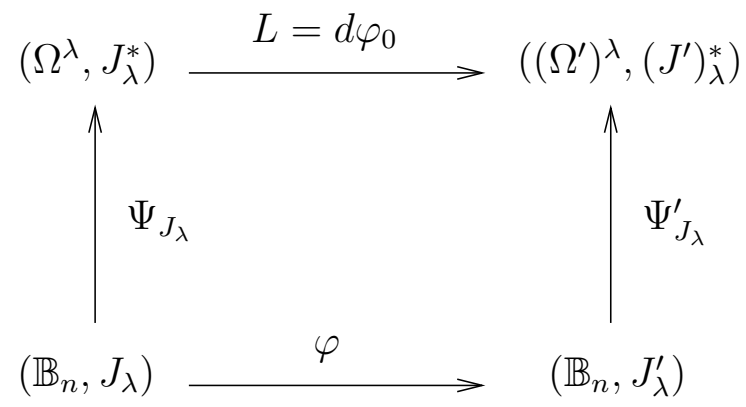

Figure 4

Conversely if $L$ satisfies condition (ii) then the map $\varphi:=\left(\Psi_{J_{\lambda}}^{\prime}\right)^{-1} \circ L \circ \Psi_{J_{\lambda}}$ satisfies condition $(i)$.

\section{KobAyASHI METRIC ON ALMOST COMPLEX MANIFOLDS}

In this section we give a lower estimate on the Kobayashi-Royden infinitesimal metric on a strictly pseudoconvex domain in an almost complex manifold. In particular, we prove that every point in an almost complex manifold has a basis of complete hyperbolic neighborhoods. These results were obtained in the paper [33].

\subsection{Localization of the Kobayashi-Royden metric.}

3.1.1. Kobayashi-Royden infinitesimal pseudometric. Let $(M, J)$ be an almost complex manifold. According to [61], for every $p \in M$ there is a neighborhood $\mathcal{V}$ of 0 in $T_{p} M$ such that for every $v \in \mathcal{V}$ there exists $f \in \mathcal{O}_{J}(\Delta, M)$ satisfying $f(0)=p, d f(0)\left(\frac{\partial}{\partial \operatorname{Re}(\zeta)}\right)=v$. This allows to define the Kobayashi-Royden infinitesimal pseudometric $K_{(M, J)}$.

Definition 3.1.1. For $p \in M$ and $v \in T_{p} M, K_{(M, J)}(p, v)$ is the infimum of the set of positive $\alpha$ such that there exists a J-holomorphic disc $f: \Delta \rightarrow M$ satisfying $f(0)=p$ and $d f(0)\left(\frac{\partial}{\partial \operatorname{Re}(\zeta)}\right)=v / \alpha$.

The following statement is an obvious consequence of the above definition :

Proposition 3.1.2. Let $f:\left(M^{\prime}, J^{\prime}\right) \rightarrow(M, J)$ be a $\left(J^{\prime}, J\right)$-holomorphic map. Then $K_{(M, J)}\left(f\left(p^{\prime}\right), d f\left(p^{\prime}\right)\left(v^{\prime}\right)\right) \leq K_{\left(M^{\prime}, J^{\prime}\right)}\left(p^{\prime}, v^{\prime}\right)$ for every $p^{\prime} \in M^{\prime}, v^{\prime} \in T_{p^{\prime}} M^{\prime}$.

We denote by $d_{(M, J)}^{K}$ the integrated pseudodistance of the Kobayashi-Royden infinitesimal pseudometric. According to the almost complex version of Royden's theorem [50, 44], the infinitesimal pseudometric is an upper semicontinuous function on the tangent bundle $T M$ of $M$ and $d_{(M, J)}^{K}$ coincides with the usual Kobayashi pseudodistance on $(M, J)$ defined by means of $J$-holomorphic discs. Similarly to the case of the integrable structure we have :

Definition 3.1.3. ( $i)$ Let $p \in M$. Then $M$ is locally hyperbolic at $p$ if there exists a neighborhood $U$ of $p$ and a positive constant $C$ such that for every $q \in U, v \in T_{q} M$ : $K_{(M, J)}(q, v) \geq C\|v\|$.

(ii) $(M, J)$ is hyperbolic if it is locally hyperbolic at every point. 
(iii) $(M, J)$ is complete hyperbolic if the Kobayashi ball $B_{(M, J)}^{K}(p, r):=\{q \in M$ : $\left.d_{(M, J)}^{K}(p, q)<r\right\}$ is relatively compact in $M$ for every $p \in M, r \geq 0$.

Lemma 3.1.4. Let $r<1$ and let $\theta_{r}$ be a smooth nondecreasing function on $\mathbb{R}^{+}$such that $\theta_{r}(s)=s$ for $s \leq r / 3$ and $\theta_{r}(s)=1$ for $s \geq 2 r / 3$. Let $(M, J)$ be an almost complex manifold, and let $p$ be a point of $M$. Then there exists a neighborhood $U$ of $p$, positive constants $A=A(r), B=B(r)$ and a diffeomorphism $z: U \rightarrow \mathbb{B}$ such that $z(p)=0, d z(p) \circ$ $J(p) \circ d z^{-1}(0)=J_{\text {st }}$ and the function $\log \left(\theta_{r}\left(|z|^{2}\right)\right)+\theta_{r}(A|z|)+B|z|^{2}$ is J-plurisubharmonic on $U$.

Proof of Lemma 3.1.4. Denote by $w$ the standard coordinates in $\mathbb{C}^{n}$. It follows from Lemma 3 that there exist positive constants $A$ and $\lambda_{0}$ such that the function $\log \left(|w|^{2}\right)+A|w|$ is $J^{\prime}$ plurisubharmonic on $\mathbb{B}$ for every almost complex structure $J^{\prime}$, defined in a neighborhood of $\overline{\mathbb{B}}$ in $\mathbb{C}^{n}$ and such that $\left\|J^{\prime}-J_{s t}\right\|_{\mathcal{C}^{2}(\overline{\mathbb{B}})} \leq \lambda_{0}$. This means that the function $v(w)=$ $\log \left(\theta_{r}\left(|w|^{2}\right)\right)+\theta_{r}(A|w|)$ is $J^{\prime}$-plurisubharmonic on $B\left(0, r^{\prime}\right)=\left\{w \in \mathbb{C}^{n}:|w|<r^{\prime}\right\}$ for every such almost complex structure $J^{\prime}$, where $r^{\prime}=\inf (\sqrt{r / 3}, r / 3 A)$. Decreasing $\lambda_{0}$ if necessary, we may assume that the function $|w|^{2}$ is strictly $J^{\prime}$-plurisubharmonic on $\mathbb{B}$. Then, since $v$ is smooth on $\mathbb{B} \backslash B\left(0, r^{\prime}\right)$, there exists a positive constant $B$ such that the function $v+B|w|^{2}$ is $J^{\prime}$-plurisubharmonic on $\mathbb{B}$ for $\left\|J^{\prime}-J_{s t}\right\|_{\mathcal{C}^{2}(\overline{\mathbb{B}})} \leq \lambda_{0}$. According to Lemma 1.1.2 there exists a neighborhood $U$ of $p$ and a diffeomorphism $z: U \rightarrow \mathbb{B}$ such that $\left\|z_{*}(J)-J_{s t}\right\|_{\mathcal{C}^{2}(\overline{\mathbb{B}})} \leq \lambda_{0}$. Then the function $v \circ z=\log \left(\theta_{r}\left(|z|^{2}\right)\right)+\theta_{r}(A|z|)+B|z|^{2}$ is $J$-plurisubharmonic on $U$.

Proposition 3.1.5. (Localization principle) Let $D$ be a domain in an almost complex manifold $(M, J)$, let $p \in \bar{D}$, let $U$ be a neighborhood of $p$ in $M$ (not necessarily contained in $D$ ) and let $z: U \rightarrow \mathbb{B}$ be the diffeomorphism given by Lemma 3.1.4. Let $u$ be a $\mathcal{C}^{2}$ function on $\bar{D}$, negative and J-plurisubharmonic on $D$. We assume that $-L \leq u<0$ on $D \cap U$ and that $u-c|z|^{2}$ is J-plurisubharmonic on $D \cap U$, where $c$ and $L$ are positive constants. Then there exist a positive constant $s$ and a neighborhood $V \subset \subset U$ of $p$, depending on $c$ and $L$ only, such that for $q \in D \cap V$ and $v \in T_{q} M$ we have the following inequality:

$$
K_{(D, J)}(q, v) \geq s K_{(D \cap U, J)}(q, v) .
$$

We note that a similar statement was obtained by F.Berteloot [6] in the integrable case. The proof is based on N.Sibony's method [72.

Proof of Proposition 3.1.5. Fix a neighborhood $V$ of $p$, relatively compact in $U$. For every $q \in V$ we consider a diffeomorphism $z_{q}$ from $U$ to $\mathbb{B}$ such that $z_{q}(q)=0$ and $\left(z_{q}\right)_{*}(J)(0)=J_{s t}$. We also may assume that the function $u-c\left|z_{q}\right|^{2}$ is $J$-plurisubharmonic on $D \cap U$.

According to Lemma 3.1.4, there exist uniform positive constants $A$ and $B$ such that the function

$$
\log \left(\theta_{r}\left(\left|z_{q}\right|^{2}\right)\right)+\theta_{r}\left(A\left|z_{q}\right|\right)+B\left|z_{q}\right|^{2}
$$


is $J$-plurisubharmonic on $U$ for every $q \in V$. Set $\tau=2 B / c$ and define, for every point $q \in V$, the function $\Psi_{q}$ by :

$$
\left\{\begin{array}{l}
\Psi_{q}(z)=\theta_{r}\left(\left|z_{q}\right|^{2}\right) \exp \left(\theta_{r}\left(A\left|z_{q}\right|\right)\right) \exp (\tau u(z)) \text { if } z \in D \cap U, \\
\Psi_{q}=\exp (1+\tau u) \text { on } D \backslash U
\end{array}\right.
$$

Then for every $0<\varepsilon \leq B$, the function $\log \left(\Psi_{q}\right)-\varepsilon\left|z_{q}\right|^{2}$ is $J$-plurisubharmonic on $D \cap U$ and hence $\Psi_{q}$ is $J$-plurisubharmonic on $D \cap U$. Since $\Psi_{q}$ coincides with $\exp (\tau u)$ outside $U$, it is globally $J$-plurisubharmonic on $D$.

Let $f: \Delta \rightarrow D$ be a $J$-holomorphic disc such that $f(0)=q \in V$ and $(\partial f / \partial R e(\zeta))(0)=$ $v / \alpha$ where $v \in T_{q} M$ and $\alpha>0$. For $\zeta$ sufficiently close to 0 we have

$$
f(\zeta)=q+d f(0)(\zeta)+\mathcal{O}\left(|\zeta|^{2}\right)
$$

Consider the function

$$
\varphi(\zeta)=\Psi_{q}(f(\zeta)) /|\zeta|^{2}
$$

which is subharmonic on $\Delta \backslash\{0\}$. Since

$$
\varphi(\zeta)=\left|z_{q}(f(\zeta))\right|^{2} /|\zeta|^{2} \exp \left(A\left|z_{q}(f(\zeta))\right|\right) \exp (\tau u(f(\zeta)))
$$

for $\zeta$ close to 0 and

$$
\left(z_{q} \circ f\right)(\zeta)=d z_{q}(q)(d f(0)(\partial / \partial \operatorname{Re}(\zeta))) \zeta+\mathcal{O}\left(|\zeta|^{2}\right)
$$

we obtain that $\lim \sup _{\zeta \rightarrow 0} \varphi(\zeta)$ is finite. Moreover

$$
\limsup _{\zeta \rightarrow 0} \varphi(\zeta) \geq \| d z_{q}(q)\left(d f(0)(\partial / \partial \operatorname{Re}(\zeta)) \|^{2} \exp (-2 B|u(q)| / c) .\right.
$$

Applying the maximum principle to a subharmonic extension of $\varphi$ on $\Delta$ we obtain the inequality

$$
\left\|d z_{q}(q)(d f(0)(\partial / \partial R e(\zeta)))\right\|^{2} \leq \exp (1+2 B|u(q)| / c) .
$$

Furthermore there exists a positive constant $C$ such that

$$
\|d f(0)(\partial / \partial \operatorname{Re}(\zeta))\|^{2} \leq C\left\|d z_{q}(q)(d f(0)(\partial / \partial \operatorname{Re}(\zeta)))\right\|^{2} .
$$

Hence, by definition of the Kobayashi-Royden infinitesimal pseudometric, we obtain for every $q \in D \cap V, v \in T_{q} M$ :

$$
K_{(D, J)}(q, v) \geq C^{-1 / 2}\left(\exp \left(-1-2 B \frac{|u(q)|}{c}\right)\right)^{1 / 2}\|v\| .
$$

Consider now the Kobayashi ball $B_{(D, J)}(q, \alpha)=\left\{w \in D: d_{(D, J)}^{K}(w, q)<\alpha\right\}$. It follows from Lemma 2.2 of [20] (whose proof is identical in the almost complex setting) that restricting $V$ if necessary we can find a positive constant $s<1$, independent of $q$, such that for every $J$-holomorphic disc $f: \Delta \rightarrow D$ satisfying $f(0) \in D \cap V$ we have $f(s \Delta) \subset D \cap U$ (see Figure 5). This gives the inequality (3.1). 


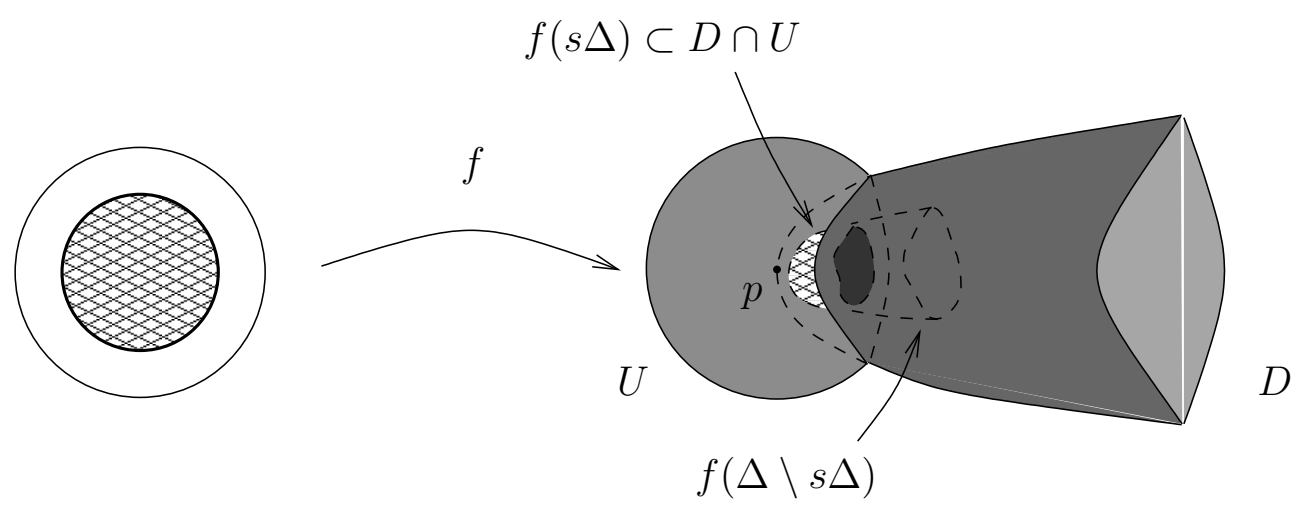

Figure 5

3.2. Uniform estimates of the Kobayashi-Royden metric. In the present section we refine the lower estimate of the Kobayashi-Royden metric.

Proposition 3.2.1. Let $D$ be a domain in an almost complex manifold $(M, J)$, let $p \in \bar{D}$, let $U$ be a neighborhood of $p$ in $M$ (not necessarily contained in $D$ ) and let $z: U \rightarrow \mathbb{B}$ be the diffeomorphism given by Lemma 3.1.4. Let $u$ be a $\mathcal{C}^{2}$ function on $\bar{D}$, negative and $J$-plurisubharmonic on $D$. We assume that $-L \leq u<0$ on $D \cap U$ and that $u-c|z|^{2}$ is $J$-plurisubharmonic on $D \cap U$, where $c$ and $L$ are positive constants. Then there exists a neighborhood $U^{\prime}$ of $p$ and a constant $c^{\prime}>0$, depending on $c$ and $L$ only, such that :

$$
K_{(D, J)}(q, v) \geq c^{\prime} \frac{\|v\|}{|u(q)|^{1 / 2}},
$$

for every $q \in D \cap U^{\prime}$ and every $v \in T_{q} M$.

Proof of proposition 3.2.1. We use the notations of the proof of Proposition 3.1.5. Consider a positive constant $r$ that will be specified later and let $\theta$ be a smooth non decreasing function on $\mathbb{R}^{+}$such that $\theta(x)=x$ for $x \leq 1 / 3$ and $\theta(x)=1$ for $x \geq 2 / 3$. Restricting $U$ if necessary we may aasume that the function $\log \left(\theta\left(\left|z_{q} / r\right|^{2}\right)\right)+A\left|z_{q}\right|+B\left|z_{q} / r\right|^{2}$ is $J$-plurisubharmonic on $D \cap U$, independently of $q$ and $r$.

Consider now the function $\Psi_{q}(z)=\theta\left(\left|z_{q}\right|^{2} / r^{2}\right) \exp \left(A\left|z_{q}\right|\right) \exp (\tau u)$ where $\tau=1 /|u(q)|$ and $r=(2 B|u(q)| / c)^{1 / 2}$. Since the function $\tau u-2 B|z / r|^{2}$ is $J$-plurisubharmonic, we may assume, shrinking $U$ if necessary, that the function $\tau u-B\left|z_{q} / r\right|^{2}$ is $J$-plurisubharmonic on $D \cap U$ for every $q \in V$. Hence the function $\log \left(\Psi_{q}\right)$ is $J$-plurisubharmonic on $D \cap U$. It follows from the estimate (3.1) that there is a positive constant $s$ such that $D_{(D, J)}(q, v) \geq s K_{(D \cap U, J)}(q, v)$ for every $q \in V, v \in T_{q} M$. Let $q \in V$, let $v \in T_{q} M$ and let $f: \Delta \rightarrow D \cap U$ be a $J_{-}$ holomorphic disc such that $f(0)=q$ and $d f(0)(\partial / \partial R e(\zeta))=v / \alpha$ where $\alpha>0$. Consider the function $\varphi(\zeta)=\Psi_{q}(f(\zeta)) /|\zeta|^{2}$ which is subharmonic on $\Delta \backslash\{0\}$. As above we obtain that $\lim \sup _{\zeta \rightarrow 0} \varphi(\zeta)$ is finite and $\lim \sup _{\zeta \rightarrow 0} \phi(\zeta) \geq\|v\|^{2} \exp (2) /\left(r^{2} \alpha^{2}\right)$. There exists a positive 
constant $C^{\prime}$, independent of $q$, such that $\left|z_{q}\right| \leq C^{\prime}$ on $U$. Applying the maximum principle to a subharmonic extension of $\varphi$ on $\Delta$, we obtain the inequality

$$
\alpha \geq \sqrt{\frac{c}{2 B \exp \left(1+A C^{\prime}\right)}}\|v\|^{2} /|u(q)|^{1 / 2} .
$$

This completes the proof.

3.2.1. Scaling and estimates of the Kobayashi-Royden metric. In this Section we present a precise lower estimate on the Kobayashi-Royden infinitesimal metric on a strictly pseudoconvex domain in $(M, J)$.

Theorem 3.2.2. Let $M$ be a real $2 n$-dimensional manifold with an almost complex structure $J$ and let $D=\{\rho<0\}$ be a relatively compact domain in $(M, J)$. We assume that $\rho$ is a $\mathcal{C}^{2}$ defining function of $D$, strictly $J$-plurisubharmonic in a neighborhood of $\bar{D}$. Then there exists a positive constant $c$ such that:

$$
K_{(D, J)}(p, v) \geq c\left[\frac{\left|\partial_{J} \rho(p)(v-i J(p) v)\right|^{2}}{|\rho(p)|^{2}}+\frac{\|v\|^{2}}{|\rho(p)|}\right]^{1 / 2},
$$

for every $p \in D$ and every $v \in T_{p} M$.

We start with the small almost complex deformations of the standard structure. In the second subsection, we consider the case of an arbitrary almost complex structure, not necessarily close to the standard one. We use non-isotropic dilations in special coordinates "reducing" an almost complex structure in order to represent a strictly pseudoconvex hypersurface on an almost complex manifold as the Siegel sphere equipped with an arbitrary small deformation of the standard structure. We stress that such a representation cannot be obtained by the isotropic dilations of Lemma 1 since the limit hypersurface is just a hyperplane.

3.2.2. Small deformations of the standard structure. We start the proof of Theorem 3.2 .2 with the following:

Proposition 3.2.3. Let $D=\{\rho<0\}$ be a bounded domain in $\mathbb{C}^{n}$, where $\rho$ is a $\mathcal{C}^{2}$ defining function of $D$, strictly $J_{\text {st }}$-plurisubharmonic in a neighborhood of $\bar{D}$. Then there exist positive constants $c$ and $\lambda_{0}$ such that for every almost complex structure $J$ defined in a neighborhood of $\bar{D}$ and such that $\left\|J-J_{\text {st }}\right\|_{\mathcal{C}^{2}(\bar{D})} \leq \lambda_{0}$ estimate (3.4) is satisfied for every $p \in D, v \in \mathbb{C}^{n}$.

Proof. We note that according to Proposition 3.1.5 (see estimate (3.2)) it is sufficient to prove the inequality near $\partial D$. Suppose by contradiction that there exists a sequence $\left(p^{\nu}\right)$ of points in $D$ converging to a boundary point $q$, a sequence $\left(v^{\nu}\right)$ of unitary vectors and a sequence $\left(J_{\nu}\right)$ of almost complex structures defined in a neighborhood of $\bar{D}$, satisfying $\left\|J_{\nu}-J_{s t}\right\|_{\mathcal{C}^{2}(\bar{D})} \rightarrow_{\nu \rightarrow \infty} 0$, such that the quotient

$$
K_{\left(D \cap U, J_{\nu}\right)}\left(p^{\nu}, v^{\nu}\right)\left[\frac{\left|\partial_{J_{\nu}} \rho\left(p^{\nu}\right)\left(v^{\nu}-i J_{\nu}\left(p^{\nu}\right) v^{\nu}\right)\right|^{2}}{\left|\rho\left(p^{\nu}\right)\right|^{2}}+\frac{\left\|v^{\nu}\right\|^{2}}{\left|\rho\left(p^{\nu}\right)\right|}\right]^{-1 / 2}
$$


tends to 0 as $\nu$ tends to $\infty$, where $U$ is a neighborhood of $q$. For sufficiently large $\nu$ denote by $\delta_{\nu}$ the euclidean distance from $p^{\nu}$ to the boundary of $D$ and by $q^{\nu} \in \partial D$ the unique point such that $\left|p^{\nu}-q^{\nu}\right|=\delta_{\nu}$. Without loss of generality we assume that $q=0$, that $T_{0}(\partial D)=\left\{z:=\left({ }^{\prime} z, z_{n}\right) \in \mathbb{C}^{n}: \operatorname{Re}\left(z_{n}\right)=0\right\}$ and that $J_{\nu}\left(q^{\nu}\right)=J_{s t}$ for every $\nu$.

Consider a sequence of biholomorphic (for the standard structure) transformations $T^{\nu}$ in a neigborhood of the origin, such that $T^{\nu}\left(q^{\nu}\right)=0$ and such that the image $D^{\nu}:=T^{\nu}(D)$ satisfies

$$
T_{0}\left(\partial D^{\nu}\right)=\left\{z \in \mathbb{C}^{n}: \operatorname{Re}\left(z_{n}\right)=0\right\} .
$$

We point out that the sequence $\left(T^{\nu}\right)_{\nu}$ converges uniformly to the identity map since $q^{\nu} \rightarrow$ $q=0$ as $\nu \rightarrow \infty$ and hence that the sequence $\left(\left(T^{\nu}\right)^{-1}\right)_{\nu}$ is bounded. We still denote by $J_{\nu}$ the direct image $\left(T^{\nu}\right)_{*}\left(J_{\nu}\right)$. Let $U_{1}$ be a neighborhood of the origin such that $\bar{U} \subset U_{1}$. For sufficiently large $\nu$ we have $T^{\nu}(U) \subset U_{1}$. We may assume that every domain $D^{\nu}$ is defined on $U_{1}$ by

$$
D^{\nu} \cap U_{1}=\left\{z \in U_{1}: \rho^{\nu}(z):=\operatorname{Re}\left(z_{n}\right)+\left.\left.\right|^{\prime} z\right|^{2}+\mathcal{O}\left(|z|^{3}\right)<0\right\},
$$

and that the sequence $\left(\hat{p}^{\nu}=T^{\nu}\left(p^{\nu}\right)=\left(0^{\prime},-\delta_{\nu}\right)\right)_{\nu}$ is on the real inward normal to $\partial D^{\nu}$ at 0 . Of course, the functions $\rho^{\nu}$ converge uniformly with all derivatives to the defining function $\rho$ of $D$. In what follows we omit the hat and write $p^{\nu}$ instead of $\hat{p}^{\nu}$.

Denote by $R$ the function

$$
R(z)=\operatorname{Re}\left(z_{n}\right)+\left.\left.\right|^{\prime} z\right|^{2}+\left(\operatorname{Re}\left(z_{n}\right)+\left.\left.\right|^{\prime} z\right|^{2}\right)^{2} .
$$

There is a neighborhood $V_{0}$ of the origin in $\mathbb{C}^{n}$ such that the function $R$ is strictly $J_{s t^{-}}$ plurisubharmonic on $V_{0}$. Fix $\alpha>0$ small enough such that the point $z^{\alpha}=\left({ }^{\prime} 0,-\alpha\right)$ belongs to $V_{0}$. Consider the dilation $\Lambda_{\nu}$ defined on $\mathbb{C}^{n}$ by $\Lambda_{\nu}(z)=\left(\left(\alpha / \delta_{\nu}\right)^{1 / 2^{\prime}} z,\left(\alpha / \delta_{\nu}\right) z_{n}\right)$. If we set $J^{\nu}:=\Lambda_{\nu} \circ J_{\nu} \circ\left(\Lambda_{\nu}\right)^{-1}$ then we have :

Lemma 3.2.4. $\lim _{\nu \rightarrow \infty} J^{\nu}=J_{\text {st }}$, uniformly on compact subsets of $\mathbb{C}^{n}$.

Proof. Considering $J$ as a matrix valued function, we may assume that the Taylor expansion of $J_{\nu}$ at the origin is given by $J_{\nu}=J_{s t}+L_{\nu}(z)+\mathcal{O}\left(|z|^{2}\right)$ on $U$, uniformly with respect to $\nu$. Hence $J^{\nu}\left(z^{0}\right)(v)=J_{s t}(v)+L_{\nu}\left({ }^{\prime} z,\left(\delta_{\nu} / \alpha\right)^{1 / 2} z_{n}\right)(v)+\mathcal{O}\left(\mid\left(\delta_{\nu} \mid\right)\|v\|\right.$. Since $\lim _{\nu \rightarrow \infty} L_{\nu}=0$ by assumption, we obtain the desired result.

Let $\tilde{\rho}^{\nu}:=\left(\alpha / \delta_{\nu}\right) \rho^{\nu} \circ \Lambda_{\nu}^{-1}$ and $G^{\nu}:=\left\{z \in \Lambda_{\nu}\left(U_{1}\right): \tilde{\rho}^{\nu}(z)<0\right\}$. Then the function $R^{\nu}:=\tilde{\rho}^{\nu}+\left(\tilde{\rho}^{\nu}\right)^{2}$ converges with all its derivatives to $R$, uniformly on compact subsets of $\mathbb{C}^{n}$. Hence $R^{\nu}$ is strictly plurisubharmonic on $V_{0}$ and according to Lemma 3.2 .4 there is a positive constant $C$ such that for sufficiently large $\nu$ the function $R^{\nu}-C|z|^{2}$ is strictly $J^{\nu}$-plurisubharmonic on $V_{0}$. Since $\sup _{z \in G^{\nu} \cap \partial V_{0}}\left(R^{\nu}(z)-C|z|^{2}\right)=-C^{\prime}<0$, the function

$$
\tilde{R}^{\nu}:=\left\{\begin{array}{lll}
R^{\nu}-C|z|^{2} & \text { on } & D^{\nu} \cap V_{0} \\
-\frac{C^{\prime}}{2} & \text { on } & D^{\nu} \backslash V_{0}
\end{array}\right.
$$

is $J^{\nu}$-plurisubharmonic on $G^{\nu}$, strictly $J^{\nu}$-plurisubharmonic on $G^{\nu} \cap V_{0}$. Since $z^{\alpha}$ belongs to $V_{0}$, it follows from the Proposition 3.1.5 (see estimate (3.2)) that there exists a positive constant $C^{\prime}>0$ such that for sufficiently large $\nu$ we have :

$$
K_{\left(G^{\nu}, J^{\nu}\right)}\left(z^{\alpha}, v\right) \geq C^{\prime}\|v\|
$$


for every $v \in \mathbb{C}^{n}$.

Moreover for $v \in \mathbb{C}^{n}$ and for sufficiently large $\nu$ we have :

$$
K_{\left(D^{\nu} \cap U_{1}, J_{\nu}\right)}\left(p^{\nu}, v\right)=K_{\left(G^{\nu}, J^{\nu}\right)}\left(z^{\alpha}, \Lambda_{\nu}(v)\right) \geq C^{\prime}\left\|\Lambda_{\nu}(v)\right\| .
$$

This gives the inequality :

$$
K_{\left(D^{\nu} \cap U, J_{\nu}\right)}\left(p^{\nu}, v\right) \geq C^{\prime}\left(\frac{\alpha\left|v_{1}\right|^{2}}{\delta_{\nu}}+\cdots+\frac{\alpha\left|v_{n-1}\right|^{2}}{\delta_{\nu}}+\frac{\alpha^{2}\left|v_{n}\right|^{2}}{\delta_{\nu}^{2}}\right)^{1 / 2} .
$$

Since $C_{1} \delta_{\nu}$ is equivalent to $\left|\rho\left(p^{\nu}\right)\right|$ as $\nu \rightarrow \infty$, we obtain that there is a positive constant $C^{\prime \prime}$ such that

$$
K_{\left(D^{\nu} \cap U, J_{\nu}\right)}\left(p^{\nu}, v\right) \geq C^{\prime \prime}\left(\frac{\|v\|^{2}}{\left|\rho\left(p^{\nu}\right)\right|}+\frac{\left|v_{n}\right|^{2}}{\left|\rho\left(p^{\nu}\right)\right|^{2}}\right)^{1 / 2} .
$$

Since $J_{\nu}(0)=J_{s t}$, we have $\left|\partial \rho\left(p^{\nu}\right)\left(v-i J_{\nu}\left(p^{\nu}\right)(v)\right)\right|^{2}=\left|\partial_{J_{s t}} \rho\left(p^{\nu}\right)(v)\right|^{2}+\mathcal{O}\left(\delta_{\nu}\right)\|v\|^{2}=$ $\left|v_{n}\right|^{2}+\mathcal{O}\left(\delta_{\nu}\right)\|v\|^{2}$. Hence there exists a positive constant $\tilde{C}$ such that

$$
K_{\left(D^{\nu} \cap U, J_{\nu}\right)}\left(p^{\nu}, v\right) \geq \tilde{C}\left(\frac{\|v\|^{2}}{\left|\rho\left(p^{\nu}\right)\right|}+\frac{\left|\partial_{J} \rho\left(p^{\nu}\right)\left(v-i J_{\nu}\left(p^{\nu}\right)(v)\right)\right|^{2}}{\left|\rho\left(p^{\nu}\right)\right|^{2}}\right)^{1 / 2},
$$

contradicting the assumption on the quotient (3.5). This proves the desired estimate.

We have the following corollary :

Corollary 3.2.5. Let $(M, J)$ be an almost complex manifold. Then every $p \in M$ has a basis of complete hyperbolic neighborhoods.

Proof. Let $p \in M$. According to Example 1 there exist a neighborhood $U$ of $p$ and a diffeomorphism $z: U \rightarrow \mathbb{B}$, centered at $p$, such that the function $|z|^{2}$ is strictly $J$-plurisubharmonic on $U$ and $\left\|z_{\star}(J)-J_{s t}\right\|_{\mathcal{C}^{2}(U)} \leq \lambda_{0}$. Hence the open ball $\left\{x \in \mathbb{C}^{n}:\|x\|<1 / 2\right\}$ equipped with the structure $z_{\star}(J)$ satisfies the hypothesis of Theorem 3.2.3. Now the estimate on the Kobaysahi-Royden metric given by this theorem implies that this ball is complete hyperbolic by the standard integration argument.

3.2.3. Arbitrary almost complex structures. We turn now to the proof of Theorem 3.2 .2 on an arbitrary strictly pseudoconvex domain in an almost complex manifold $(M, J)(J$ is not supposed to be a small deformation of the standard structure). In view of Proposition 3.1.5 it suffices to prove the statement in a neighborhood $U$ of a boundary point $q \in \partial D$. Considering local coordinates $z$ centered at $q$, we may assume that $D \cap U$ is a domain in $\mathbb{C}^{n}$ and $0 \in \partial D$, $J(0)=J_{s t}$. The idea of the proof is to reduce the situation to the case of a small deformation of the standard structure considered in Proposition 3.2.3. In the case of real dimension four Theorem 3.2 .2 is a direct corollary of Proposition 3.2.3. In the case of arbitrary dimension the proof of Theorem 3.2.2 requires a slight modification of Proposition 3.2.3. So we treat this case seperately.

3.2.4. Case where $\operatorname{dim} M=4$. According to [73] Corollary 3.1.2, there exist a neighborhood $U$ of $q$ in $M$ and complex coordinates $z=\left(z_{1}, z_{2}\right): U \rightarrow \mathbb{B}_{2} \subset \mathbb{C}^{2}, z(0)=0$ such that $z_{*}(J)(0)=J_{s t}$ and moreover, a map $f: \Delta \rightarrow \mathbb{B}$ is $J^{\prime}:=z_{*}(J)$-holomorphic if it satisfies the equations 


$$
\frac{\partial f_{j}}{\partial \bar{\zeta}}=A_{j}\left(f_{1}, f_{2}\right) \overline{\left(\frac{\partial f_{j}}{\partial \zeta}\right)}, j=1,2
$$

where $A_{j}(z)=O(|z|), j=1,2$.

As pointed out before, one can obtain such coordinates by considering two transversal foliations of $\mathbb{B}$ by $J^{\prime}$-holomorphic curves and then taking these curves into the lines $z_{j}=$ const by a local diffeomorphism (see Figure 1). The direct image of the almost complex structure $J$ under such a diffeomorphism has a diagonal matrix $J^{\prime}\left(z_{1}, z_{2}\right)=\left(a_{j k}(z)\right)_{j k}$ with $a_{12}=a_{21}=0$ and $a_{j j}=i+\alpha_{j j}$ where $\alpha_{j j}(z)=\mathcal{O}(|z|)$ for $j=1,2$. We point out that the lines $z_{j}=$ const are $J$-holomorphic after a suitable parametrization (which, in general, is not linear).

In what follows we omit the prime and denote this structure again by $J$. We may assume that the complex tangent space $T_{0}(\partial D) \cap J(0) T_{0}(\partial D)=T_{0}(\partial D) \cap i T_{0}(\partial D)$ is given by $\left\{z_{2}=0\right\}$. In particular, we have the following expansion for the defining function $\rho$ of $D$ on $U: \rho(z, \bar{z})=2 \operatorname{Re}\left(z_{2}\right)+2 \operatorname{Re} K(z)+H(z)+\mathcal{O}\left(|z|^{3}\right)$, where $K(z)=\sum k_{\nu \mu} z_{\nu} z_{\mu}, k_{\nu \mu}=k_{\mu \nu}$ and $H(z)=\sum h_{\nu \mu} z_{\nu} \bar{z}_{\mu}, h_{\nu \mu}=\bar{h}_{\mu \nu}$.

Consider the non-isotropic dilations $\Lambda_{\delta}:\left(z_{1}, z_{2}\right) \mapsto\left(\delta^{-1 / 2} z_{1}, \delta^{-1} z_{2}\right)=\left(w_{1}, w_{2}\right)$ with $\delta>0$. If $J$ has the above diagonal form in the coordinates $\left(z_{1}, z_{2}\right)$ in $\mathbb{C}^{2}$, then its direct image $J_{\delta}=\left(\Lambda_{\delta}\right)_{*}(J)$ has the form $J_{\delta}\left(w_{1}, w_{2}\right)=\left(a_{j k}\left(\delta^{1 / 2} w_{1}, \delta w_{2}\right)\right)_{j k}$ and so $J_{\delta}$ tends to $J_{s t}$ in the $\mathcal{C}^{2}$ norm as $\delta \rightarrow 0$. On the other hand, $\partial D$ is, in the coordinates $w$, the zero set of the function $\rho_{\delta}=\delta^{-1}\left(\rho \circ \Lambda_{\delta}^{-1}\right)$. As $\delta \rightarrow 0$, the function $\rho_{\delta}$ tends to the function $2 \operatorname{Rew} w_{2}+2 \operatorname{ReK}\left(w_{1}, 0\right)+$ $H\left(w_{1}, 0\right)$ which defines a $J_{s t^{-}}$strictly pseudoconvex domain by Lemma 1.3.7. So we may apply Proposition 3.2.3. This proves Theorem 3.2.2 in dimension 4.

3.2.5. Case where $\operatorname{dim} M=2 n$. In this case we cannot apply directly Proposition 3.2 .3 since $J$ can not be deformed by the non-isotropic dilations to the standard structure. Instead we use the invariance of the Levi form with respect to the non-isotropic dilations.

We suppose that in a neighborhhod of the origin we have $J=J_{s t}+\mathcal{O}(|z|)$. We also may assume that in these coordinates the defining function $\rho$ of $D$ has the form $\rho=2 R e z_{n}+$ $2 \operatorname{Re} K(z)+H(z)+\mathcal{O}\left(|z|^{3}\right)$, where $K$ and $H$ are defined similarly to the 4-dimensional case and $\rho$ is strictly $J$-plurisubharmonic at the origin. We use the notation $z=\left({ }^{\prime} z, z_{n}\right)$.

Consider the non-isotropic dilations $\Lambda_{\delta}:\left({ }^{\prime} z, z_{n}\right) \mapsto\left(w^{\prime}, w_{n}\right)=\left(\delta^{-1 / 2^{\prime}} z, \delta^{-1} z_{n}\right)$ and set $J_{\delta}=\left(\Lambda_{\delta}\right)_{*}(J)$. Then $J_{\delta}$ tends to the almost complex structure $J_{0}(z)=J_{s t}+L\left({ }^{\prime} z, 0\right)$ where $L\left({ }^{\prime} z, 0\right)=\left(L_{k j}\left({ }^{\prime} z, 0\right)\right)_{k j}$ denotes a matrix with $L_{k j}=0$ for $k=1, \ldots, n-1, j=1, \ldots, n$, $L_{n n}=0$ and $L_{n j}\left({ }^{\prime} z, 0\right), j=1, \ldots, n-1$ being (real) linear forms in ' $z$.

Let $\rho_{\delta}=\delta^{-1}\left(\rho \circ \Lambda_{\delta}^{-1}\right)$. As $\delta \rightarrow 0$, the function $\rho_{\delta}$ tends to the function $\tilde{\rho}=$ $2 R e z_{n}+2 \operatorname{ReK}\left({ }^{\prime} z, 0\right)+H\left({ }^{\prime} z, 0\right)$ in the $\mathcal{C}^{2}$ norm. By the invariance of the Levi form we have $\mathcal{L}^{J}(\rho)(0)\left(\Lambda_{\delta}^{-1}(v)\right)=\mathcal{L}^{J_{\delta}}\left(\rho \circ \Lambda_{\delta}^{-1}\right)(0)(v)$. Since $\rho$ is strictly $J$-plurisubharmonic, multiplying by $\delta^{-1}$ and passing to the limit at the right side as $\delta \longrightarrow 0$, we obtain that $\mathcal{L}^{J_{0}}(\tilde{\rho})(0)(v) \geq 0$ for any $v$. Now let $v=\left({ }^{\prime} v, 0\right)$. Then $\Lambda_{\delta}^{-1}(v)=\delta^{1 / 2} v$ and so $\mathcal{L}^{J}(\rho)(0)(v)=\mathcal{L}^{J_{\delta}}\left(\rho_{\delta}\right)(0)(v)$. Passing to the limit as $\delta$ tends to zero, we obtain that $\mathcal{L}^{J_{0}}(\tilde{\rho})(0)(v)>0$ for any $v=\left({ }^{\prime} v, 0\right)$

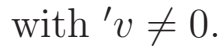

Consider now the function $R=\tilde{\rho}+\tilde{\rho}^{2}$. Then $\mathcal{L}^{J_{0}}(R)(0)(v)=\mathcal{L}^{J_{0}}(\tilde{\rho})(0)(v)+2 v_{n} \bar{v}_{n}$, so $R$ is strictly $J_{0}$-plurisubharmonic in a neighborhood of the origin. Thus the functions $R^{\nu}$ used 
in the proof of Proposition 3.2 .3 are strictly $J^{\nu}$-plurisubharmonic and their Levi forms are bounded from below by a positive constant independent of $\nu$. This allows to use Proposition 3.1.5 and the proof can be proceeded quite similarly to the proof of Proposition 3.2.3 without any changes.

3.2.6. Upper estimate of the Kobayashi-Royden metric. In this subsection we prove the following :

Theorem 3.2.6. Let $M$ be a real $2 n$-dimensional manifold with an almost complex structure $J$ and let $D=\{\rho<0\}$ be a relatively compact domain in $(M, J)$. We assume that $\rho$ is a $\mathcal{C}^{2}$ defining function of $D$, strictly J-plurisubharmonic in a neighborhood of $\bar{D}$. Then there exists a positive constant $c$ such that:

$$
K_{(D, J)}(p, v) \leq c\left[\frac{\left|\partial_{J} \rho(p)(v-i J(p) v)\right|^{2}}{|\rho(p)|^{2}}+\frac{\|v\|^{2}}{|\rho(p)|}\right]^{1 / 2},
$$

for every $p \in D$ and every $v \in T_{p} M$.

Since the estimates are purely local we may assume that $D \subset \mathbb{C}^{n}$. Let $p \in D$ be sufficiently close to $\partial D$ and let $\|v\|=1$. We choose coordinates $z=\left(z^{1}, \ldots, z^{n}\right)$ on $\mathbb{C}^{n}$ such that $0 \in \partial D$, $p=\left(0^{\prime},-\delta\right)$ where $0<\delta<1, \operatorname{dist}(p, \partial D)=\operatorname{dist}(0, p)$, and $D^{\prime}:=\left\{z \in B(0,2) / \operatorname{Re}\left(z_{n}\right)+\right.$ $\left.|z|^{2}<0\right\} \subset D \cap B(0,2)$. Moreover we may assume that the map $f_{v}: \Delta \rightarrow \mathbb{C}^{n}$ defined by $f_{v}(\zeta)=\zeta v$ is $J$-holomorphic. We denote by $d$ the distance from $p$ to $\partial D^{\prime}$ along the line $\mathbb{C} v$. Since $f_{v}(d \Delta)$ is contained in $D^{\prime}$ we have the following inequalities :

$$
K_{(D, J)}(p, v) \leq K_{\left(D^{\prime}, J\right)}(p, v) \leq \frac{1}{d}
$$

Moreover by the strict convexity of $D^{\prime}$ we have :

$$
d \geq \frac{1}{2}\left(\delta^{2}\left\|v_{n}\right\|^{2}+\delta\left\|v^{\prime}\right\|^{2} \mid\right)^{1 / 2} .
$$

On the other hand

$$
\frac{1}{\left(\delta^{2}\left\|v_{n}\right\|^{2}+\delta\left\|v^{\prime}\right\|^{2} \mid\right)^{1 / 2}} \leq\left(\frac{\left\|v_{n}\right\|^{2}}{\delta^{2}}+\frac{\left\|v^{\prime}\right\|^{2}}{\delta}\right)^{1 / 2}
$$

which implies the desired statement.

3.3. Boundary continuity and localization of biholomorphisms. In this section we give some technical results necessary for the proof of the Fefferman mapping theorem. They are also of independent interest.

3.3.1. Boundary continuity of diffeomorphisms. Using estimates of the Kobayashi-Royden metric together with the boundary distance preserving property, we obtain, by means of classical arguments (see, for instance, K.Diederich-J.E.Fornaess [27]), the following

Theorem 3.3.1. Let $D$ and $D^{\prime}$ be two smoothly relatively compact strictly pseudoconvex domains in almost complex manifolds $(M, J)$ and $\left(M^{\prime}, J^{\prime}\right)$ respectively. Let $f: D \rightarrow D^{\prime}$ be a smooth diffeomorphism biholomorphic with respect to $J$ and $J^{\prime}$. Then $f$ extends as a 1/2-Hölder homeomorphism between the closures of $D$ and $D^{\prime}$. 
We recall the following estimates of the Kobayashi-Royden infinitesimal metric obtained previously :

Lemma 3.3.2. Let $D$ be a relatively compact strictly pseudoconvex domain in an almost complex manifold $(M, J)$. Then there exists a constant $C>0$ such that

$$
(1 / C)\|v\| / \operatorname{dist}(p, \partial D)^{1 / 2} \leq K_{(D, J)}(p, v) \leq C\|v\| / \operatorname{dist}(p, \partial D)
$$

for every $p \in D$ and $v \in T_{p} M$.

Proof of Theorem 3.3.1. For any $p \in D$ and any tangent vector $v$ at $p$ we have by Lemma 3.3.2:

$$
C_{1} \frac{\left\|d f_{p}(v)\right\|}{\operatorname{dist}\left(f(p), \partial D^{\prime}\right)^{1 / 2}} \leq K_{\left(D^{\prime}, J^{\prime}\right)}\left(f(p), d f_{p}(v)\right)=K_{(D, J)}(p, v) \leq C_{2} \frac{\|v\|}{\operatorname{dist}(p, \partial D)}
$$

which implies, by Proposition 1.4.8, the estimate

$$
\left\|d f_{p}\right\| \leq C \frac{\|v\|}{\operatorname{dist}(p, \partial D)^{1 / 2}} .
$$

This gives the desired statement.

Theorem 3.3.1 allows to reduce the proof of Fefferman's theorem to a local situation. Indeed, let $p$ be a boundary point of $D$ and $f(p)=p^{\prime} \in \partial D^{\prime}$. It suffices to prove that $f$ extends smoothly to a neighborhood of $p$ on $\partial D$. Consider coordinates $z$ and $z^{\prime}$ defined in small neighborhoods $U$ of $p$ and $U^{\prime}$ of $p^{\prime}$ respectively, with $U^{\prime} \cap D^{\prime}=f(D \cap U)$ (this is possible since $f$ extends as a homeomorphism at $p)$. We obtain the following situation. If $\Gamma=z(\partial D \cap U)$ and $\Gamma^{\prime}=z^{\prime}\left(\partial D^{\prime} \cap U^{\prime}\right)$ then the map $z^{\prime} \circ f \circ z^{-1}$ is defined on $z(D \cap U)$ in $\mathbb{C}^{2}$, continuous up to the hypersurface $\Gamma$ with $f(\Gamma) \subset \Gamma^{\prime}$. Furthermore the map $z^{\prime} \circ f \circ z^{-1}$ is a diffeomorphism between $z(D \cap U)$ and $z^{\prime}\left(D^{\prime} \cap U^{\prime}\right)$ and the hypersurfaces $\Gamma$ and $\Gamma^{\prime}$ are strictly pseudoconvex for the structures $z_{*}(J)$ and $\left(z^{\prime}\right)_{*}\left(J^{\prime}\right)$ respectively. Finally, we may choose $z$ and $z^{\prime}$ such that $z_{*}(J)$ and $z_{*}^{\prime}\left(J^{\prime}\right)$ are represented by diagonal matrix functions in the coordinates $z$ and $z^{\prime}$. As we proved in Lemma 1.3.7. $\Gamma$ (resp. $\Gamma^{\prime}$ ) is also strictly $J_{s t}$-psdeudoconvex at the origin. We call such coordinates $z$ (resp. $z^{\prime}$ ) canonical coordinates at $p$ (resp. at $p^{\prime}$ ). Using the non-isotropic dilation as in Section 2.5, we may assume that the norms $\left\|z_{*}(J)-J_{s t}\right\|_{\mathcal{C}^{2}}$ and $\left\|z_{*}^{\prime}\left(J^{\prime}\right)-J_{s t}\right\|_{\mathcal{C}^{2}}$ are as small as needed. This localization is crucially used in the sequel and we write $J$ (resp. $J^{\prime}$ ) instead of $z_{*}(J)$ (resp. $z_{*}^{\prime}\left(J^{\prime}\right)$ ); we identify $f$ with $z^{\prime} \circ f \circ z^{-1}$.

3.3.2. Hölder extension of holomorphic discs. We study the boundary continuity of pseudoholomorphic discs attached to smooth totally real submanifolds in almost complex manifolds.

Recall that in the case of the integrable structure every smooth totally real submanifold $E$ (of maximal dimension) is the zero set of a positive strictly plurisubharmonic function of class $\mathcal{C}^{2}$. This remains true in the almost complex case. Indeed, we can choose coordinates $z$ in a neighborhood $U$ of $p \in E$ such that $z(p)=0, z_{*}(J)=J_{s t}+O(|z|)$ on $U$ and $z(E \cap U)=\left\{w=(x, y) \in z(U): r_{j}(w)=x^{j}+o(|w|)=0\right\}$. The function $\rho=\sum_{j=1}^{n} r_{j}^{2}$ is strictly $J_{s t}$-plurisubharmonic on $z(U)$ and so remains strictly $z_{*}(J)$-plurisubharmonic, 
restricting $U$ if necessary. Covering $E$ by such neighborhoods, we conclude by mean of the partition of unity.

Let $f: \Delta \rightarrow M$ be a $J$-holomorphic disc and let $\gamma$ be an open arc on the unit circle $\partial \Delta$. As usual we denote by $C(f, \gamma)$ the cluster set of $f$ on $\gamma$; this consists of points $p \in M$ such that $p=\lim _{k \rightarrow \infty} f\left(\zeta_{k}\right)$ for a sequence $\left(\zeta_{k}\right)_{k}$ in $\Delta$ converging to a point in $\gamma$.

Theorem 3.3.3. Let $G$ be a relatively compact domain in an almost complex manifold $(M, J)$ and let $\rho$ be a strictly J-plurisubharmonic function of class $\mathcal{C}^{2}$ on $\bar{G}$. Let $f: \Delta \rightarrow G$ be a $J$-holomorphic disc such that $\rho \circ f \geq 0$ on $\Delta$. Suppose that $\gamma$ is an open non-empty arc on $\partial \Delta$ such that the cluster set $C(f, \gamma)$ is contained in the zero set of $\rho$. Then $f$ extends as a Hölder 1/2-continuous map on $\Delta \cup \gamma$.

We begin the proof by the following well-known assertion (see, for instance, 4]).

Lemma 3.3.4. Let $\phi$ be a positive subharmonic function in $\Delta$ such that the measures $\mu_{r}\left(e^{i \theta}\right):=\phi\left(r e^{i \theta}\right) d \theta$ converge in the weak-star topology to a measure $\mu$ on $\partial \Delta$ as $r \rightarrow 1$. Suppose that $\mu$ vanishes on an open arc $\gamma \subset \partial \Delta$. Then for every compact subset $K \subset \Delta \cup \gamma$ there exists a constant $C>0$ such that $\phi(\zeta) \leq C(1-|\zeta|)$ for any $\zeta \in K \cup \Delta$.

Now fix a point $a \in \gamma$, a constant $\delta>0$ small enough so that the intersection $\gamma \cap(a+\delta \bar{\Delta})$ is compact in $\gamma$; we denote by $\Omega_{\delta}$ the intersection $\Delta \cap(a+\delta \Delta)$. By Lemma 3.3.4, there exists a constant $C>0$ such that, for any $\zeta$ in $\Omega_{\delta}$, we have

$$
\rho \circ f(\zeta) \leq C(1-|\zeta|)
$$

Let $\left(\zeta_{k}\right)_{k}$ be a sequence of points in $\Delta$ converging to $a$ with $\lim _{k \rightarrow \infty} f\left(\zeta_{k}\right)=p$. By assumption, the function $\rho$ is strictly $J$-plurisubharmonic in a neighborhood $U$ of $p$; hence there is a constant $\varepsilon>0$ such that the function $\rho-\varepsilon|z|^{2}$ is $J$-plurisubharmonic on $U$.

Lemma 3.3.5. There exists a constant $A>0$ with the following property: If $\zeta$ is an arbitrary point of $\Omega_{\delta / 2}$ such that $f(\zeta)$ is in $G \cap z^{-1}(\mathbb{B})$, then $\left\|\left|d f_{\zeta} \|\right| \leq A(1-|\zeta|)^{-1 / 2}\right.$.

Proof of Lemma 3.3.5. Set $d=1-|\zeta|$; then the disc $\zeta+d \Delta$ is contained in $\Omega_{\delta}$. Define the domain $G_{d}=\{w \in G: \rho(w)<2 C d\}$. Then it follows by (3.8) that the image $f(\zeta+$ $d \Delta$ ) is contained in $G_{d}$, where the $J$-plurisubharmonic function $u_{d}=\rho-2 C d$ is negative. Moreover we have lower estimates on the Kobayashi-Royden infinitesimal pseudometric given by Proposition 3.2.1,

Hence there exists a positive constant $M$ (independent of $d$ ) such that $K_{\left(G_{d}, J\right)}(w, \eta) \geq$ $M|\eta|\left|u_{d}(w)\right|^{-1 / 2}$, for any $w$ in $G \cap z^{-1}(\mathbb{B})$ and any $\eta \in T_{w} \Omega$. On another hand, we have $K_{\zeta+d \Delta}(\zeta, \tau)=|\tau| / d$ for any $\tau$ in $T_{\zeta} \Delta$ indentified with $\mathbb{C}$. By the decreasing property of the Kobayashi-Royden metric, for any $\tau$ we have

$$
M\left\|d f_{\zeta}(\tau)\right\|\left|u_{d}(f(\zeta))\right|^{-1 / 2} \leq K_{\left(G_{d}, J\right)}\left(f(\zeta), d f_{\zeta}(\tau)\right) \leq K_{\zeta+d \Delta}(\zeta, \tau)=|\tau| / d .
$$

Therefore, ||$\left|d f_{\zeta}\right||| \leq M^{-1}\left|u_{d}(f(\zeta))\right|^{1 / 2} / d$. As $-2 C d \leq u_{d}(f(\zeta))<0$, this implies the desired statement in Lemma 3.3.5 with $A=M^{-1}(2 C)^{1 / 2}$. 
Proof of Theorem 3.3.3. Lemma 3.3.5 implies that $f$ extends as a $1 / 2$-Hölder map to a neighborhood of the point $a$ in view of an integration argument inspired by the classical Hardy-Littlewood theorem. This proves Theorem 3.3 .3 .

\section{NonisotropiC SCALING OF ALMOST COMPLEX MANIFOLDS With BOUNDARY}

We consider a biholomorphism $f$ between two relatively compact, strictly pseudoconvex domains $D$ and $D^{\prime}$ in almost complex manifolds $(M, J)$ and $\left(M^{\prime}, J^{\prime}\right)$. The aim of this Section is to provide a precise information about the boundary behavior of the tangent map of $f$. For convenience of the reader, we begin this Section with the case of four real dimensional almost complex manifolds (subsections 4.1 and 4.2). The case of higher dimension will be treated in Subsection 4.3 .

4.1. Localization and boundary behavior of the tangent map. Our considerations being purely local, we can assume that $D$ and $D^{\prime}$ are domains in $\mathbb{C}^{2}, \Gamma$ and $\Gamma^{\prime}$ are open smooth pieces of their boundaries containing the origin, the almost complex structure $J$ (resp. $\left.J^{\prime}\right)$ is defined in a neighborhood of $D$ (resp. $\left.D^{\prime}\right), f$ is a $\left(J, J^{\prime}\right)$ biholomorphism from $D$ to $D^{\prime}$, continuous up to $\Gamma, f(\Gamma)=\Gamma^{\prime}, f(0)=0$. The matrix $J$ (resp. $J^{\prime}$ ) is diagonal on $D$ (resp. $D^{\prime}$ ).

Consider a basis $\left(\omega_{1}, \omega_{2}\right)$ of $(1,0)$ differential forms (for the structure $J$ ) in a neighborhood of the origin. Since $J$ is diagonal, we may choose $\omega_{j}=d z^{j}-B_{j}(z) d \bar{z}^{j}, j=1,2$. Denote by $Y=\left(Y_{1}, Y_{2}\right)$ the corresponding dual basis of $(1,0)$ vector fields. Then $Y_{j}=\partial / \partial z^{j}-$ $\beta_{j}(z) \partial / \bar{\partial} z^{j}, j=1,2$. Here $\beta_{j}(0)=\beta_{k}(0)=0$. The basis $Y(0)$ simply coincides with the canonical $(1,0)$ basis of $\mathbb{C}^{2}$. In particular $Y_{1}(0)$ is a basis vector of the holomorphic tangent space $H_{0}^{J}(\partial D)$ and $Y_{2}(0)$ is "normal" to $\partial D$. Consider now for $t \geq 0$ the translation $\partial D-t$ of the boundary of $D$ near the origin. Consider, in a neighborhood of the origin, a $(1,0)$ vector field $X_{1}$ (for $J$ ) such that $X_{1}(0)=Y_{1}(0)$ and $X_{1}(z)$ generates the complex tangent space $H_{z}^{J}(\partial D-t)$ at every point $z \in \partial D-t, 0 \leq t<<1$. Setting $X_{2}=Y_{2}$, we obtain a basis of vector fields $X=\left(X_{1}, X_{2}\right)$ on $D$ (restricting $D$ if necessary). Any complex tangent vector $v \in T_{z}^{(1,0)}(D, J)$ at point $z \in D$ admits the unique decomposition $v=v_{t}+v_{n}$ where $v_{t}=\alpha_{1} X_{1}(z)$ (the tangent component) and $v_{n}=\alpha_{2} X_{2}(z)$ (the normal component). Identifying $T_{z}^{(1,0)}(D, J)$ with $T_{z} D$ we may consider the decomposition $v=v_{t}+v_{n}$ for $v \in T_{z}(D)$. Finally we consider this decomposition for points $z$ in a neighborhood of the boundary.

We fix a $(1,0)$ basis vector fields $X$ (resp. $\left.X^{\prime}\right)$ on $D\left(\right.$ resp. $\left.D^{\prime}\right)$ as above.

Proposition 4.1.1. The matrix $A=\left(A_{k j}\right)_{k, j=1,2}$ of the differential $d f_{z}$ with respect to the bases $X(z)$ and $X^{\prime}(f(z))$ satisfies the following estimates : $A_{11}=O(1), A_{12}=$ $O\left(\operatorname{dist}(z, \partial D)^{-1 / 2}\right), A_{21}=O\left(\operatorname{dist}(z, \partial D)^{1 / 2}\right)$ and $A_{22}=O(1)$. 
Proof of Proposition 4.1.1. Consider the case where $v=v_{t}$. It follows from the estimates of the Kobayashi-Royden metric obtained previously that:

$$
\begin{aligned}
\frac{1}{C}\left(\frac{\left\|\left(d f_{z}\left(v_{t}\right)\right)_{t}\right\|}{\operatorname{dist}\left(f(z), \partial D^{\prime}\right)^{1 / 2}}+\frac{\left\|\left(d f_{z}\left(v_{t}\right)\right)_{n}\right\|}{\operatorname{dist}\left(f(z), \partial D^{\prime}\right)}\right) & \leq K_{\left(D^{\prime}, J^{\prime}\right)}\left(f(z), d f_{z}\left(v_{t}\right)\right) \\
& =\quad K_{(D, J)}\left(z, v_{t}\right) \leq C \frac{\left\|v_{t}\right\|}{\operatorname{dist}(z, \partial D)^{1 / 2}} .
\end{aligned}
$$

This implies that $\left\|\left(d f_{z}\left(v_{t}\right)\right)_{t}\right\| \leq C^{5 / 2}\left\|v_{t}\right\|$ and $\left\|\left(d f_{z}\left(v_{t}\right)\right)_{n}\right\| \leq C^{3} \operatorname{dist}(z, \partial D)^{1 / 2}\left\|v_{t}\right\|$, by the boundary distance preserving property given in Proposition 1.4.8. We obtain the estimates for the normal component in a similar way.

4.2. Non isotropic dilations. Our goal now is to prove Fefferman's mapping theorem without the assumption of $\mathcal{C}^{1}$-smoothness of $f$ up to the boundary. This requires an application of the estimates of the Kobayashi-Royden metric given above and the scaling method due to S.Pinchuk; we adapt this to the almost complex case.

We reduced the problem to the following local situation. Let $D$ and $D^{\prime}$ be domains in $\mathbb{C}^{2}, \Gamma$ and $\Gamma^{\prime}$ be open $\mathcal{C}^{\infty}$-smooth pieces of their boundaries, containing the origin. We assume that an almost complex structure $J$ is defined and $\mathcal{C}^{\infty}$-smooth in a neighborhood of the closure $\bar{D}, J(0)=J_{s t}$ and $J$ has a diagonal form in a neighborhood of the origin: $J(z)=\operatorname{diag}\left(a_{11}(z), a_{22}(z)\right)$. Similarly, we assume that $J^{\prime}$ is diagonal in a neighborhood of the origin, $J^{\prime}(z)=\operatorname{diag}\left(a_{11}^{\prime}(z), a_{22}^{\prime}(z)\right)$ and $J^{\prime}(0)=J_{s t}$. The hypersurface $\Gamma\left(\operatorname{resp} . \Gamma^{\prime}\right)$ is supposed to be strictly $J$-pseudoconvex (resp. strictly $J^{\prime}$-pseudoconvex). Finally, we assume that $f: D \rightarrow D^{\prime}$ is a $\left(J, J^{\prime}\right)$-biholomorphic map, 1/2-Hölder homeomorphism between $D \cup \Gamma$ and $D^{\prime} \cup \Gamma^{\prime}$, such that $f(\Gamma)=\Gamma^{\prime}$ and $f(0)=0$. Finally, $\Gamma$ may be defined in a neighborhood of the origin by the equation $\rho(z)=0$ where $\rho(z)=2 \operatorname{Re} z^{2}+2 \operatorname{Re} K(z)+H(z)+o\left(|z|^{2}\right)$ and $K(z)=\sum K_{\mu \nu} z^{\mu \nu}, H(z)=\sum h_{\mu \nu} z^{\mu} \bar{z}^{\nu}, k_{\mu \nu}=k_{\nu \mu}, h_{\mu \nu}=\bar{h}_{\nu \mu}$. The crucial point is that $H\left(z^{1}, 0\right)$ is a positive Hermitian form on $\mathbb{C}$, meaning that in these coordinates $\Gamma$ is strictly pseudoconvex at the origin with respect to the standard structure of $\mathbb{C}^{2}$ (see Lemma 1.3.7]for the proof). Of course, $\Gamma^{\prime}$ admits a similar local representation. In what follows we assume that we are in this setting.

Let $\left(p^{k}\right)$ be a sequence of points in $D$ converging to 0 and let $\Sigma:=\left\{z \in \mathbb{C}^{2}: 2 R e z^{2}+\right.$ $\left.2 \operatorname{Re} K\left(z^{1}, 0\right)+H\left(z^{1}, 0\right)<0\right\}, \Sigma^{\prime}:=\left\{z \in \mathbb{C}^{2}: 2 \operatorname{Re} z^{2}+2 \operatorname{Re} K^{\prime}\left(z^{1}, 0\right)+H^{\prime}\left(z^{1}, 0\right)<0\right\}$. The scaling procedure associates with the pair $\left(f,\left(p^{k}\right)_{k}\right)$ a biholomorphism $\phi$ (with respect to the standard structure $J_{s t}$ ) between $\Sigma$ and $\Sigma^{\prime}$. Since $\phi$ is obtained as a limit of a sequence of biholomorphic maps conjugated with $f$, some of their properties are related and this can be used to study boundary properties of $f$ and to prove that its cotangent lift is continuous up to the conormal bundle $\Sigma(\partial D)$.

4.2.1. Fixing suitable local coordinates and dilations. For any boundary point $t \in \partial D$ we consider the change of variables $\alpha^{t}$ defined by

$$
\left(z^{1}\right)^{*}=\frac{\partial \rho}{\partial \bar{z}^{2}}(t)\left(z^{1}-t^{1}\right)-\frac{\partial \rho}{\partial \bar{z}^{1}}(t)\left(z^{2}-t^{2}\right),\left(z^{2}\right)^{*}=\sum_{j=1}^{2} \frac{\partial \rho}{\partial z^{j}}(t)\left(z^{j}-t^{j}\right) .
$$


Then $\alpha^{t}$ maps $t$ to 0 . The real normal at 0 to $\Gamma$ is mapped by $\alpha^{t}$ to the line $\left\{z^{1}=0, y_{2}=0\right\}$. For every $k$, we denote by $t^{k}$ the projection of $p^{k}$ onto $\partial D$ and by $\alpha^{k}$ the change of variables $\alpha^{t}$ with $t=t^{k}$. Set $\delta_{k}=\operatorname{dist}\left(p^{k}, \Gamma\right)$. Then $\alpha^{k}\left(p^{k}\right)=\left(0,-\delta_{k}\right)$ and $\alpha^{k}(D)=\left\{2 \operatorname{Re} z^{2}+\right.$ $\left.O\left(|z|^{2}\right)<0\right\}$ near the origin. Since the sequence $\left(\alpha^{k}\right)_{k}$ converges to the identity map, the sequence $\left(\alpha^{k}\right)_{*}(J)$ of almost complex structures tends to $J$ as $k \rightarrow \infty$. Moreover there is a sequence $\left(L^{k}\right)$ of linear automorphisms of $\mathbb{R}^{4}$ such that $\left(L^{k} \circ \alpha^{k}\right)_{*}(J)(0)=J_{s t}$. Then $\left(L^{k} \circ \alpha^{k}\right)\left(p^{k}\right)=\left(o\left(\delta_{k}\right),-\delta_{k}^{\prime}\right)$ with $\delta_{k}^{\prime} \sim \delta_{k}$ and $\left(L^{k} \circ \alpha^{k}\right)(D)=\left\{\operatorname{Re}\left(z^{2}+\tau_{k} z^{1}\right)+O\left(|z|^{2}\right)<0\right\}$ near the origin, with $\tau_{k}=o(1)$. Hence there is sequence $\left(M^{k}\right)$ of $\mathbb{C}$-linear transformations of $\mathbb{C}^{2}$, converging to the identity, such that $\left(T^{k}:=M^{k} \circ L^{k} \circ \alpha^{k}\right)$ is a sequence of linear transformations converging to the identity, and $D^{k}:=T^{k}(D)$ is defined near the origin by $D^{k}=\left\{\rho_{k}(z)=R e z^{2}+O\left(|z|^{2}\right)<0\right\}$. Finally $\tilde{p}_{k}=T^{k}\left(p^{k}\right)=\left(o\left(\delta_{k}\right), \delta_{k}^{\prime \prime}+i o\left(\delta_{k}\right)\right)$ with $\delta_{k}^{\prime \prime} \sim \delta_{k}$. We also denote by $\Gamma^{k}=\left\{\rho_{k}=0\right\}$ the image of $\Gamma$ under $T^{k}$. Furthermore, the sequence of almost complex structures $\left(J_{k}:=\left(T^{k}\right)_{*}(J)\right)$ converges to $J$ as $k \rightarrow \infty$ and $J_{k}(0)=J_{s t}$.

We proceed quite similarly for the target domain $D^{\prime}$. For $s \in \Gamma^{\prime}$ we define the transformation $\beta^{s}$ by

$$
\left(z^{1}\right)^{*}=\frac{\partial \rho^{\prime}}{\partial \bar{z}^{2}}(s)\left(z^{1}-s^{1}\right)-\frac{\partial \rho^{\prime}}{\partial \bar{z}^{1}}(s)\left(z^{2}-s^{2}\right),\left(z^{2}\right)^{*}=\sum_{j=1}^{2} \frac{\partial \rho^{\prime}}{\partial z^{j}}(s)\left(z^{j}-s^{j}\right) .
$$

Let $s^{k}$ be the projection of $q^{k}:=f\left(p^{k}\right)$ onto $\Gamma^{\prime}$ and let $\beta^{k}$ be the corresponding map $\beta^{s}$ with $s=s^{k}$. The sequence $\left(q^{k}\right)$ converges to $0=f(0)$ so $\beta^{k}$ tends to the identity. Considering linear transformations $\left(L^{\prime}\right)^{k}$ and $\left(M^{\prime}\right)^{k}$, we obtain a sequence $\left(T^{\prime k}\right)$ of linear transformations converging to the identity and satisfying the following properties. The domain $\left(D^{k}\right)^{\prime}:=$ $T^{\prime k}\left(D^{\prime}\right)$ is defined near the origin by $\left(D^{k}\right)^{\prime}=\left\{\rho_{k}^{\prime}(z):=\operatorname{Re} z^{2}+O\left(|z|^{2}\right)<0\right\}, \Gamma_{k}^{\prime}=\left\{\rho_{k}^{\prime}=0\right\}$ and $\tilde{q}_{k}=T^{\prime k}\left(q^{k}\right)=\left(o\left(\varepsilon_{k}\right), \varepsilon_{k}^{\prime \prime}+i o\left(\varepsilon_{k}\right)\right)$ with $\varepsilon_{k}^{\prime \prime} \sim \varepsilon_{k}$, where $\varepsilon_{k}=\operatorname{dist}\left(q^{k}, \Gamma^{\prime}\right)$. The sequence of almost complex structures $\left(J_{k}^{\prime}:=\left(T^{\prime k}\right)_{*}\left(J^{\prime}\right)\right)$ converges to $J^{\prime}$ as $k \rightarrow \infty$ and $J_{k}^{\prime}(0)=J_{s t}$.

Finally, the map $f^{k}:=T^{\prime k} \circ f \circ\left(T^{k}\right)^{-1}$ satisfies $f^{k}\left(\tilde{p}_{k}\right)=\tilde{q}_{k}$ and is a biholomorphism between the domains $D^{k}$ and $\left(D^{\prime}\right)^{k}$ with respect to the almost complex structures $J_{k}$ and $J_{k}^{\prime}$.

Consider now the non isotropic dilations $\phi_{k}:\left(z^{1}, z^{2}\right) \mapsto\left(\delta_{k}^{1 / 2} z^{1}, \delta_{k} z^{2}\right)$ and $\psi_{k}\left(z^{1}, z^{2}\right)=$ $\left(\varepsilon_{k}^{1 / 2} z^{1}, \varepsilon_{k} z^{2}\right)$ and set $\hat{f}^{k}=\left(\psi_{k}\right)^{-1} \circ f^{k} \circ \phi_{k}$. Then the map $\hat{f}^{k}$ is biholomorphic with respect to the almost complex structures $\hat{J}_{k}:=\left(\left(\phi_{k}\right)^{-1}\right)_{*}\left(J_{k}\right)$ and $\hat{J}_{k}^{\prime}:=\left(\psi_{k}^{-1}\right)_{*}\left(J_{k}^{\prime}\right)$. Moreover if $\hat{D}^{k}:=\phi_{k}^{-1}\left(D^{k}\right)$ and $\left(\hat{D}^{\prime}\right)^{k}:=\psi_{k}^{-1}\left(\left(D^{\prime}\right)^{k}\right)$ then $\hat{D}^{k}=\left\{z \in \phi_{k}^{-1}(U): \hat{\rho}_{k}(z)<0\right\}$ where

$$
\begin{aligned}
\hat{\rho}_{k}(z) & :=\delta_{k}^{-1} \rho\left(\phi_{k}(z)\right) \\
& =2 \operatorname{Re} z^{2}+\delta_{k}^{-1}\left[2 \operatorname{ReK}\left(\delta_{k}^{1 / 2} z^{1}, \delta_{k} z^{2}\right)+H\left(\delta_{k}^{1 / 2} z^{1}, \delta_{k} z^{2}\right)+o\left(\left|\left(\delta_{k}^{1 / 2} z^{1}, \delta_{k} z^{2}\right)\right|^{2}\right) .\right.
\end{aligned}
$$

and $\left(\hat{D}^{\prime}\right)^{k}=\left\{z \in \phi_{k}^{-1}(U): \hat{\rho}_{k}^{\prime}(z)<0\right\}$ where

$$
\begin{aligned}
\hat{\rho}_{k}^{\prime}(z) & :=\varepsilon_{k}^{-1} \rho^{\prime}\left(\psi_{k}(z)\right) \\
& =2 \operatorname{Re} z^{2}+\varepsilon_{k}^{-1}\left[2 \operatorname{Re} K^{\prime}\left(\varepsilon_{k}^{1 / 2} z^{1}, \varepsilon_{k} z^{2}\right)+H^{\prime}\left(\varepsilon_{k}^{1 / 2} z^{1}, \varepsilon_{k} z^{2}\right)+o\left(\left|\left(\varepsilon_{k}^{1 / 2} z^{1}, \varepsilon_{k} z^{2}\right)\right|^{2}\right) .\right.
\end{aligned}
$$

Since $U$ is a fixed neighborhood of the origin, the pullbacks $\phi_{k}^{-1}(U)$ tend to $\mathbb{C}^{2}$ and the functions $\hat{\rho}_{k}$ tend to $\hat{\rho}(z)=2 \operatorname{Re} z^{2}+2 \operatorname{Re} K\left(z^{1}, 0\right)+H\left(z^{1}, 0\right)$ in the $\mathcal{C}^{2}$ norm on any compact subset of $\mathbb{C}^{2}$. Similarly, since $U^{\prime}$ is a fixed neighborhood of the origin, the pullbacks $\psi_{k}^{-1}\left(U^{\prime}\right)$ tend to $\mathbb{C}^{2}$ and the functions $\hat{\rho}_{k}^{\prime}$ tend to $\hat{\rho}^{\prime}(z)=2 \operatorname{Re} z^{2}+2 \operatorname{Re} K^{\prime}\left(z^{1}, 0\right)+H^{\prime}\left(z^{1}, 0\right)$ in the $\mathcal{C}^{2}$ 
norm on any compact subset of $\mathbb{C}^{2}$. If $\Sigma:=\left\{z \in \mathbb{C}^{2}: \hat{\rho}(z)<0\right\}$ and $\Sigma^{\prime}:=\left\{z \in \mathbb{C}^{2}: \hat{\rho}^{\prime}(z)<\right.$ $0\}$ then the sequence of points $\hat{p}^{k}=\phi_{k}^{-1}\left(\tilde{p}_{k}\right) \in \hat{D}^{k}$ converges to the point $(0,-1) \in \Sigma$ and the sequence of points $\hat{q}^{k}=\psi_{k}^{-1}\left(\tilde{q}^{k}\right) \in \hat{D}^{\prime}{ }^{k}$ converges to $(0,-1) \in \Sigma^{\prime}$. Finally $\hat{f}^{k}\left(\hat{p}^{k}\right)=\hat{q}^{k}$.

\subsubsection{Convergence of the dilated families. We begin with the following}

Lemma 4.2.1. The sequences $\left(\hat{J}_{k}^{\prime}\right)$ and $\left(\hat{J}_{k}\right)$ of almost complex structures converge to the standard structure uniformly (with all partial derivatives of any order) on compact subsets of $\mathbb{C}^{2}$.

Proof of Lemma 4.2.1. Denote by $a_{\nu \mu}^{k}(z)$ the elements of the matrix $J_{k}$. Since $J_{k} \rightarrow J$ and $J$ is diagonal, we have $a_{\nu \mu}^{k} \rightarrow a_{\nu \mu}$ for $\nu=\mu$ and $a_{\nu \mu}^{k} \rightarrow 0$ for $\nu \neq \mu$. Moreover, since $J_{k}(0)=J_{s t}, a_{\nu \mu}^{k}(0)=i$ for $\nu=\mu$ and $a_{\nu \mu}(0)=0$ for $\nu \neq \mu$. The elements $\hat{a}_{\nu \mu}^{k}$ of the matrix $\hat{J}_{k}$ are given by: $\hat{a}_{\nu \mu}^{k}\left(z^{1}, z^{2}\right)=a_{\nu \mu}^{k}\left(\delta_{k}^{1 / 2} z^{1}, \delta_{k} z^{2}\right)$ for $\nu=\mu, \hat{a}_{12}^{k}\left(z^{1}, z^{2}\right)=\delta_{k}^{1 / 2} a\left(\delta_{k}^{1 / 2} z^{1}, \delta_{k} z^{2}\right)$ and $\hat{a}_{21}^{k}\left(z^{1}, z^{2}\right)=\delta_{k}^{-1 / 2} a_{21}^{k}\left(\delta_{k}^{1 / 2} z^{1}, \delta_{k} z^{2}\right)$. This implies the desired result.

The next statement is crucial.

Proposition 4.2.2. The sequence $\left(\hat{f}^{k}\right)$ (together with all derivatives) is a relatively compact family (with respect to the compact open topology) on $\Sigma$; every cluster point $\hat{f}$ is a biholomorphism (with respect to $J_{\text {st }}$ ) between $\Sigma$ and $\Sigma^{\prime}$, satisfying $\hat{f}(0,-1)=(0,-1$ ) and $\left(\partial \hat{f}^{2} / \partial z^{2}\right)(0,-1)=1$.

Proof of Proposition 4.2.2. Step 1: convergence. Our proof is based on the method developed by F.Berteloot-G.Coeuré [8]. Consider a domain $G \subset \mathbb{C}^{2}$ of the form $G=\{z \in W: \lambda(z)=$ $\left.2 \operatorname{Re} z^{2}+2 \operatorname{Re} K(z)+H(z)+o\left(|z|^{2}\right)<0\right\}$ where $W$ is a neighborhood of the origin. We assume that an almost complex structure $J$ is diagonal on $W$ and that the hypersurface $\{\lambda=0\}$ is strictly $J$-pseudoconvex at any point. Given $a \in \mathbb{C}^{2}$ and $\delta>0$ denote by $Q(a, \delta)$ the non-isotropic ball $Q(a, \delta)=\left\{z:\left|z^{1}-a_{1}\right|<\delta^{1 / 2},\left|z^{2}-a_{2}\right|<\delta\right\}$. Denote also by $d_{\delta}$ the non-isotropic dilation $d_{\delta}\left(z^{1}, z^{2}\right)=\left(\delta^{-1 / 2} z^{1}, \delta^{-1} z^{2}\right)$.

Lemma 4.2.3. There exist positive constants $\delta_{0}, C, r$ satisfying the following property : for every $\delta \leq \delta_{0}$ and for every J-holomorphic disc $g: \Delta \rightarrow G$ such that $g(0) \in Q(0, \delta)$ we have the inclusion $g(r \Delta) \subset Q(0, C \delta)$.

Proof of Lemma 4.2.3. Assume by contradiction that there exist positive sequences $\delta_{k} \rightarrow 0$, $C_{k} \rightarrow+\infty$, a sequence $\zeta_{k} \in \Delta, \zeta_{k} \rightarrow 0$ and a sequence $g_{k}: \Delta \rightarrow G$ of $J$-holomorphic discs such that $g_{k}(0) \in Q\left(0, \delta_{k}\right)$ and $g_{k}\left(\zeta_{k}\right) \notin Q\left(0, C_{k} \delta_{k}\right)$. Denote by $d_{k}$ the dilations $d_{\delta}$ with $\delta=\delta_{k}$ and consider the composition $h_{k}=d_{k} \circ g_{k}$ defined on $\Delta$. The dilated domains $G_{k}:=d_{k}(G)$ are defined by $\left\{z \in d_{k}(W): \lambda_{k}(z):=\delta_{k}^{-1} \lambda \circ d_{k}^{-1}(z)<0\right\}$ and the sequence $\left(\lambda_{k}\right)$ converges uniformly on compact subsets of $\mathbb{C}^{2}$ to $\hat{\lambda}: z \mapsto 2 \operatorname{Re} z^{2}+2 \operatorname{Re} K(z)+H\left(z^{1}, 0\right)$. Since $J$ is diagonal, the sequence of structures $J_{k}:=\left(d_{k}\right)_{*}(J)$ converges to $J_{s t}$ in the $\mathcal{C}^{2}$ norm on compact subsets of $\mathbb{C}^{2}$.

The discs $h_{k}$ are $J_{k}$-holomorphic and the sequence $\left(h_{k}(0)\right)$ is contained in $Q(0,1)$; passing to a subsequence we may assume that this converges to a point $p \in \overline{Q(0,1)}$. On the other hand, the function $\hat{\lambda}+A \hat{\lambda}^{2}$ is strictly $J_{s t}$-plurisubharmonic on $Q(0,5)$ for a suitable constant $A>0$. 
Since the structures $J_{k}$ tend to $J_{s t}$, the functions $\lambda_{k}+A \lambda_{k}^{2}$ are strictly $J_{k}$-plurisubharmonic on $Q(0,4)$ for every $k$ large enough and their Levi forms admit a uniform lower bound with respect to $k$. By Proposition 3.2.1 the Kobayashi-Royden infinitesimal pseudometric on $G_{k}$ admits the following lower bound : $K_{G_{k}}(z, v) \geq C|v|$ for any $z \in G_{k} \cap Q(0,3), v \in \mathbb{C}^{2}$, with a positive constant $C$ independent of $k$. Therefore, there exists a constant $C^{\prime}>0$ such that $\left\|\left|\left(d h_{k}\right)_{\zeta} \|\right| \leq C^{\prime}\right.$ for any $\zeta \in(1 / 2) \Delta$ satisfying $h_{k}(\zeta) \in G_{k} \cap Q(0,3)$. On the other hand, the sequence $\left(\left|h_{k}\left(\zeta_{k}\right)\right|\right)$ tends to $+\infty$. Denote by $\left[0, \zeta_{k}\right]$ the segment (in $\mathbb{C}$ ) joining the origin and $\zeta_{k}$ and let $\zeta_{k}^{\prime} \in\left[0, \zeta_{k}\right]$ be the point the closest to the origin such that $h_{k}\left(\left[0, \zeta_{k}^{\prime}\right]\right) \subset G_{k} \cap \overline{Q(0,2)}$ and $h_{k}\left(\zeta_{k}^{\prime}\right) \in \partial Q(0,2)$. Since $h_{k}(0) \in Q(0,1)$, we have $\left|h_{k}(0)-h_{k}\left(\zeta_{k}^{\prime}\right)\right| \geq C^{\prime \prime}$ for some constant $C^{\prime \prime}>0$. Let $\left.\zeta_{k}^{\prime}=r_{k} e^{i \theta_{k}}, r_{k} \in\right] 0,1[$. Then

$$
\left|h_{k}(0)-h_{k}\left(\zeta_{k}^{\prime}\right)\right| \leq \int_{0}^{r_{k}}\left|\left\|\left(d h_{k}\right)\left(t e^{i \theta_{k}}\right) \mid\right\| d t \leq C^{\prime} r_{k} \rightarrow 0\right.
$$

This contradiction proves Lemma 4.2.3.

The statement of Lemma 4.2 .3 remains true if we replace the unit disc $\Delta$ by the unit ball $\mathbb{B}_{2}$ in $\mathbb{C}^{2}$ equipped with an almost complex structure $\tilde{J}$ close enough (in the $\mathcal{C}^{2}$ norm) to $J_{s t}$. For the proof it is sufficient to foliate $\mathbb{B}_{2}$ by $\tilde{J}$-holomorphic curves through the origin (in view of a smooth dependence on small perturbations of $J_{s t}$ such a foliation is a small perturbation of the foliation by complex lines through the origin and apply Lemma 4.2 .3 to the foliation.

As a corollary we have the following

Lemma 4.2.4. Let $(M, \tilde{J})$ be an almost complex manifold and let $F^{k}: M \rightarrow G$ be a sequence of $(\tilde{J}, J)$-holomorphic maps. Assume that for some point $p^{0} \in M$ we have $F^{k}(p)=\left(0,-\delta_{k}\right)$, $\delta_{k} \rightarrow 0$, and that the sequence $\left(F^{k}\right)$ converges to 0 uniformly on compact subsets of $M$. Consider the rescaled maps $d_{k} \circ F^{k}$. Then for any compact subset $K \subset M$ the sequence of norms $\left(\left\|d_{k} \circ F^{k}\right\|_{\mathcal{C}^{0}(K)}\right)$ is bounded.

Proof of Lemma 4.2.4. It is sufficient to consider a covering of a compact subset of $M$ by sufficiently small balls, similarly to [8], p.84. Indeed, consider a covering of $K$ by the balls $p^{j}+r \mathbb{B}, j=0, \ldots, N$ where $r$ is given by Lemma 4.2.3 and $p^{j+1} \in p^{j}+r \mathbb{B}$ for any $j$. For $k$ large enough, we obtain that $F^{k}\left(p^{0}+r \mathbb{B}\right) \subset Q\left(0,2 C \delta_{k}\right)$, and $F^{k}\left(p^{1}+r \mathbb{B}\right) \subset$ $Q\left(0,4 C^{2} \delta_{k}\right)$. Continuing this process we obtain that $F^{k}\left(p^{N}+r \mathbb{B}\right) \subset Q\left(0,2^{N} C^{N} \delta_{k}\right)$. This proves Lemma 4.2.4.

Now we return to the proof of Proposition 4.2.2. Lemma 4.2.4 implies that the sequence $\left(\hat{f}^{k}\right)$ is bounded (in the $\mathcal{C}^{0}$ norm) on any compact subset $K$ of $\Sigma$. Covering $K$ by small bidiscs, consider two transversal foliations by $J$-holomorphic curves on every bidisc. Since the restriction of $\hat{f}^{k}$ on every such curve is uniformly bounded in the $\mathcal{C}^{0}$-norm, it follows by the elliptic estimates that this is bounded in $\mathcal{C}^{l}$ norm for every $l$ (see [73]). Since the bounds are uniform with respect to curves, the sequence $\left(\hat{f}^{k}\right)$ is bounded in every $\mathcal{C}^{l}$-norm. So the family $\left(\hat{f}^{k}\right)$ is relatively compact.

Step 2: Holomorphicity of the limit maps. Let $\left(\hat{f}^{k_{s}}\right)$ be a subsequence converging to a smooth map $\hat{f}$. Since $f^{k_{s}}$ satisfies the holomorphicity condition $\hat{J}_{k_{s}}^{\prime} \circ d \hat{f}^{k_{s}}=d \hat{f}^{k_{s}} \circ J_{k_{s}}$, since 
$\hat{J}_{k_{s}}$ and $\hat{J}_{k_{s}}^{\prime}$ converge to $J_{s t}$, we obtain, passing to the limit in the holomorphicity condition, that $\hat{f}$ is holomorphic with respect to $J_{s t}$.

Step 3: Biholomorphicity of $\hat{f}$. Since $\hat{f}(0,-1)=(0,-1) \in \Sigma^{\prime}$ and $\Sigma^{\prime}$ is defined by a plurisubharmonic function, it follows by the maximum principle that $\hat{f}(\Sigma) \subset \Sigma^{\prime}$ (and not just a subset of $\left.\bar{\Sigma}^{\prime}\right)$. Applying a similar argument to the sequence $\left(\hat{f}^{k}\right)^{-1}$ of inverse map, we obtain that this converges (after extraction of a subsequence) to the inverse of $\hat{f}$.

Finally the domain $\Sigma$ (resp. $\Sigma^{\prime}$ ) is biholomorphic to $\mathbb{H}$ by means of the transformation $\left(z^{1}, z^{2}\right) \mapsto\left(z^{1}, z^{2}+K\left(z^{1}, 0\right)\right)$ (resp. $\left.\left(z^{1}, z^{2}\right) \mapsto\left(z^{1}, z^{2}+K^{\prime}\left(z^{1}, 0\right)\right)\right)$. Since a biholomorphism of $\mathbb{H}$ fixing the point $(0,-1)$ has the form $\left(e^{i \theta} z^{1}, z^{2}\right)$ (see, for instance, [22]), $\hat{f}$ is conjugated to this transformation by the above quadratic biholomorphisms of $\mathbb{C}^{2}$. Hence :

$$
\frac{\partial \hat{f}^{2}}{\partial z^{2}}(0,-1)=1 .
$$

This property will be used in the next Section.

4.2.3. Boundary behavior of the tangent map. We suppose that we are in the local situation described at the beginning of the previous section. Here we prove two statements concerning the boundary behavior of the tangent map of $f$ near $\Gamma$. They are obvious if $f$ is of class $\mathcal{C}^{1}$ up to $\Gamma$. In the general situation, their proofs require the scaling method of the previous section. Let $p \in \Gamma$. After a local change of coordinates $z$ we may assume that $p=0, J(0)=J_{s t}$ and $J$ is assumed to be diagonal. In the $z$ coordinates, we consider a base $X$ of $(1,0)$ (with respect to $J$ ) vector fields defined previously. Recall that $X_{2}=\partial / \partial z^{2}+a(z) \partial / \bar{\partial} z^{2}$, $a(0)=0, X_{1}(0)=\partial / \partial z^{1}$ and at every point $z^{0}, X_{1}\left(z^{0}\right)$ generates the holomorphic tangent space $H_{z}^{J}(\partial D-t), t \geq 0$. If we return to the initial coordinates and move the point $p \in \Gamma$, we obtain for every $p$ a basis $X_{p}$ of $(1,0)$ vector fields, defined in a neighborhood of $p$. Similarly, we define the basis $X_{q}^{\prime}$ for $q \in \partial D^{\prime}$.

The elements of the matrix of the tangent map $d f_{z}$ in the bases $X_{p}(z)$ and $X_{f(p)}^{\prime}(z)$ are denoted by $A_{j s}(p, z)$. According to Proposition 4.1.1 the function $A_{22}(p, \cdot)$ is upper bounded on $D$.

Proposition 4.2.5. We have:

(a) Every cluster point of the function $z \mapsto A_{22}(p, z)$ (in the notation of Proposition 4.1.1) is real when $z$ tends to a point $p \in \partial D$.

(b) For $z \in D$, let $p \in \Gamma$ such that $|z-p|=\operatorname{dist}(z, \Gamma)$. There exists a constant $A$, independent of $z \in D$, such that $\left|A_{22}(p, z)\right| \geq A$.

The proof of these statements use the above scaling construction. So we use the notations of the previous section.

Proof of Proposition 4.2.5. (a) Suppose that there exists a sequence of points $\left(p^{k}\right)$ converging to a boundary point $p$ such that $A_{22}(p, \cdot)$ tends to a complex number $a$. Applying the above scaling construction, we obtain a sequence of maps $\left(\hat{f}^{k}\right)_{k}$. Consider the two basis $\hat{X}^{k}:=$ $\left.\delta_{k}^{1 / 2}\left(\left(\phi_{k}^{-1}\right) \circ T^{k}\right)\left(X_{1}\right), \delta_{k}\left(\left(\phi_{k}^{-1}\right) \circ T^{k}\right)\left(X_{2}\right)\right)$ and $\left(\hat{X}^{\prime}\right)^{k}:=\left(\varepsilon_{k}^{-1 / 2}\left(\left(\psi_{k}^{-1}\right) \circ T^{\prime k}\right)\left(X_{1}^{\prime}\right), \varepsilon_{k}^{-1}\left(\left(\psi_{k}^{-1}\right) \circ\right.\right.$ $\left.\left.T^{\prime k}\right)\left(X_{2}^{\prime}\right)\right)$. These vector fields tend to the standard $(1,0)$ vector field base of $\mathbb{C}^{2}$ as $k$ tends to 
$\infty$. Denote by $\hat{A}_{j s}^{k}$ the elements of the matrix of $d \hat{f}^{k}(0,-1)$. Then $A_{22}^{k} \rightarrow\left(\partial \hat{f}^{2} / \partial z^{2}\right)(0,-1)=$ 1 , according to (4.1). On the other hand, $A_{22}^{k}=\varepsilon_{k}^{-1} \delta_{k} A_{22}$ and tends to $a$ by the boundary distance preserving property (Proposition 1.4.8). This gives the statement.

(b) Suppose that there is a sequence of points $\left(p^{k}\right)$ converging to the boundary such that $A_{22}$ tends to 0 . Repeating precisely the argument of (a), we obtain that $\left(\partial \hat{f}^{2} / \partial z^{2}\right)(0,-1)=0$; this contradicts (4.1).

In order to establish the next proposition, it is convenient to associate a wedge with the totally real part of the conormal bundle $\Sigma_{J}(\partial D)$ of $\partial D$ as edge. Consider in $\mathbb{R}^{4} \times \mathbb{R}^{4}$ the set $S=\left\{(z, L): \operatorname{dist}\left((z, L), \Sigma_{J}(\partial D)\right) \leq \operatorname{dist}(z, \partial D), z \in D\right\}$. Then, in a neighborhood $U$ of any totally real point of $\Sigma_{J}(\partial D)$, the set $\mathrm{S}$ contains a wedge $W_{U}$ with $\Sigma_{J}(\partial D) \cap U$ as totally real edge.

Proposition 4.2.6. Let $K$ be a compact subset of the totally real part of the conormal bundle $\Sigma_{J}(\partial D)$. Then the cluster set of the cotangent lift $\tilde{f}$ of $f$ on the conormal bundle $\Sigma(\partial D)$, when $(z, L)$ tends to $\Sigma_{J}(\partial D)$ along the wedge $W_{U}$, is relatively compactly contained in the totally real part of $\Sigma\left(\partial D^{\prime}\right)$.

Proof of Proposition 4.2.6. Let $\left(z^{k}, L^{k}\right)$ be a sequence in $W_{U}$ converging to $\left(0, \partial_{J} \rho(0)\right)=$ $\left(0, d z^{2}\right)$. Set $g=f^{-1}$. We shall prove that the sequence of linear forms $Q^{k}:={ }^{t} d g\left(w^{k}\right) L^{k}$, where $w^{k}=f\left(z^{k}\right)$, converges to a linear form which up to a real factor (in view of Part (a) of Proposition 4.2.5) coincides with $\partial_{J} \rho^{\prime}(0)=d z^{2}$ (we recall that ${ }^{t}$ denotes the transposed map). It is sufficient to prove that the first component of $Q^{k}$ with respect to the dual basis $\left(\omega_{1}, \omega_{2}\right)$ of $X$ tends to 0 and the second one is bounded below from the origin as $k$ tend to infinity. The map $X$ being of class $\mathcal{C}^{1}$ we can replace $X(0)$ by $X\left(w^{k}\right)$. Since $\left(z^{k}, L^{k}\right) \in W_{U}$, we have $L^{k}=\omega_{2}\left(z^{k}\right)+O\left(\delta_{k}\right)$, where $\delta_{k}$ is the distance from $z^{k}$ to the boundary. Since $\left\|d g_{w^{k}}\right\|==0\left(\delta_{k}^{-1 / 2}\right)$, we have $Q^{k}={ }^{t} d g_{w^{k}}\left(\omega_{2}\left(z^{k}\right)\right)+O\left(\delta_{k}^{1 / 2}\right)$. By Proposition 4.1.1, the components of ${ }^{t} d g_{w^{k}}\left(\omega_{2}\left(z^{k}\right)\right)$ with respect to the basis $\left(\omega_{1}\left(z^{k}\right), \omega_{2}\left(z^{k}\right)\right)$ are the elements of the second line of the matrix $d g_{w^{k}}$ with respect to the basis $X^{\prime}\left(w^{k}\right)$ and $X\left(z^{k}\right)$. So its first component is $0\left(\delta_{k}^{1 / 2}\right)$ and tends to 0 as $k$ tends to infinity. Finally the component $A_{22}^{k}$ is bounded below from the origin by Part (b) of Proposition 4.2.5.

4.3. Lifts of biholomorphisms to the cotangent bundle : the case of arbitrary dimension. Our further considerations rely deeply on the estimates of the Kobayashi-Royden infinitesimal pseudometric given in Section 3.

Let $D\left(\right.$ resp. $\left.D^{\prime}\right)$ be a strictly pseudoconvex domain in an almost complex manifold $(M, J)$ (resp. $\left.\left(M^{\prime}, J^{\prime}\right)\right)$ and let $f$ be a $\left(J, J^{\prime}\right)$-biholomorphism from $D$ to $D^{\prime}$. Fix a point $p \in \partial D$ and a sequence $\left(p^{k}\right)_{k}$ in $D$ converging to $p$. After extraction we may assume that the sequence $\left(f\left(p^{k}\right)\right)_{k}$ converges to a point $p^{\prime}$ in $\partial D^{\prime}$. According to the Hopf lemma, $f$ has the boundary distance property. Namely, there is a positive constant $C$ such that

$$
(1 / A) \operatorname{dist}\left(f\left(p^{k}\right), \partial D^{\prime}\right) \leq \operatorname{dist}\left(p^{k}, \partial D\right) \leq A \operatorname{dist}\left(f\left(p^{k}\right), \partial D^{\prime}\right)
$$

where $A$ is independent of $k$ (see [24]).

Since all our considerations are local we set $p=p^{\prime}=0 \in \mathbb{C}^{n}$. We may assume that $J(0)=J_{s t}$ and $J^{\prime}(0)=J_{s t}$. Let $U$ (resp. $V$ ) be a neighborhood of the origin in $\mathbb{C}^{n}$ such that $D \cap U=\left\{z \in U: \rho(z, \bar{z}):=z_{n}+\bar{z}_{n}+\operatorname{Re}(K(z))+H(z, \bar{z})+\cdots<0\right\}$ (resp. $D^{\prime} \cap V=$ 
$\left.\left\{w \in V: \rho^{\prime}(w, \bar{w}):=w_{n}+\bar{w}_{n}+\operatorname{Re}\left(K^{\prime}(w)\right)+H^{\prime}(w, \bar{w})+\cdots<0\right\}\right)$ where $K(z)=\sum k_{\nu \mu} z^{\nu} z^{\mu}$, $k_{\nu \mu}=k_{\mu \nu}, H(z)=\sum h_{\nu \mu} z^{\nu} \bar{z}^{\mu}, h_{\nu \mu}=\bar{h}_{\mu \nu}$ and $\rho$ is a strictly $J$-plurisubharmonic function on $U$ (resp. $K^{\prime}(z)=\sum k_{\nu \mu}^{\prime} w^{\nu} w^{\mu}, k_{\nu \mu}^{\prime}=k_{\mu \nu}^{\prime}, H^{\prime}(w)=\sum h_{\nu \mu}^{\prime} w^{\nu} \bar{w}^{\mu}, h_{\nu \mu}^{\prime}=\bar{h}_{\mu \nu}^{\prime}$ and $\rho^{\prime}$ is a strictly $J^{\prime}$-plurisubharmonic function on $V$ ).

4.3.1. Asymptotic behaviour of the tangent map of $f$. We wish to understand the limit behaviour (when $k \rightarrow \infty$ ) of $d f\left(p^{k}\right)$. Consider the vector fields

$$
v^{j}:=\left(\partial \rho / \partial x^{n}\right) \partial / \partial x^{j}-\left(\partial \rho / \partial x^{j}\right) \partial / \partial x^{n}
$$

for $j=1, \ldots, n-1$, and

$$
v^{n}:=\left(\partial \rho / \partial x^{n}\right) \partial / \partial y^{n}-\left(\partial \rho / \partial y^{n}\right) \partial / \partial x^{n} .
$$

Restricting $U$ if necessary, the vector fields $X^{1}, \ldots, X^{n-1}$ defined by $X^{j}:=v^{j}-i J\left(v^{j}\right)$ form a basis of the $J$-holomorphic tangent space to $\{\rho=\rho(z)\}$ at any $z \in U$. Moreover, if $X^{n}:=v^{n}-i J v^{n}$ then the family $X:=\left(X^{1}, \ldots, X^{n}\right)$ forms a basis of $(1,0)$ vector fields on $U$. Similarly we define a basis $X^{\prime}:=\left(X^{\prime 1}, \ldots, X^{\prime n}\right)$ of $(1,0)$ vector fields on $V$ such that $\left(X^{\prime 1}(w), \ldots, X^{\prime n-1}(w)\right)$ defines a basis of the $J^{\prime}$-holomorphic tangent space to $\left\{\rho^{\prime}=\rho^{\prime}(w)\right\}$ at any $w \in V$. We denote by $A\left(p^{k}\right):=\left(A\left(p^{k}\right)_{j, l}\right)_{1 \leq j, l \leq n}$ the matrix of the map $d f\left(p^{k}\right)$ in the basis $X\left(p^{k}\right)$ and $X\left(f\left(p^{k}\right)\right)$.

Remark 4.3.1. In sake of completeness we should write $X_{0}$ and $X_{0}^{\prime}$ to emphasize that the structure was normalized by the condition $J(0)=J_{s t}$ and $A\left(0, p^{k}\right)$ for $A\left(p^{k}\right)$. The same construction is valid for any boundary point of $D$. The corresponding notations will be used in Proposition 4.3.5.

Proposition 4.3.2. The matrix $A\left(p^{k}\right)$ satisfies the following estimates:

$$
A\left(p^{k}\right)=\left(\begin{array}{cc}
O_{n-1, n-1}(1) & O_{n-1,1}\left(\operatorname{dist}\left(p^{k}, \partial D\right)^{-1 / 2}\right) \\
O_{1, n-1}\left(\operatorname{dist}\left(p^{k}, \partial D\right)^{1 / 2}\right) & O_{1,1}(1)
\end{array}\right) .
$$

The matrix notation means that the following estimates are satisfied : $A\left(p^{k}\right)_{j, l}=O(1)$ for $1 \leq j, l \leq n-1, A\left(p^{k}\right)_{j, n}=O\left(\operatorname{dist}\left(p^{k}, \partial D\right)^{-1 / 2}\right)$ for $1 \leq j \leq n-1, A\left(p^{k}\right)_{n, l}=$ $O\left(\operatorname{dist}\left(p^{k}, \partial D\right)^{1 / 2}\right)$ for $1 \leq l \leq n-1$ and $A\left(p^{k}\right)_{n, n}=O(1)$.

The proof of Proposition 4.3.2 was given previously in dimension two (Proposition 4.1.1) but is valid without any modification in any dimension. We note that the asymptotic behaviour of $A\left(p^{k}\right)$ depends only on the distance from the point to $\partial D$, not on the choice of the sequence $\left(p^{k}\right)_{k}$.

4.3.2. Scaling process and model domains. The following construction is similar to the two dimensional case. For every $k$ denote by $q^{k}$ the projection of $p^{k}$ to $\partial D$ and consider the change of variables $\alpha^{k}$ defined by

$$
\left\{\begin{aligned}
\left(z^{j}\right)^{*} & =\frac{\partial \rho}{\partial \bar{z}^{n}}\left(q^{k}\right)\left(z^{j}-\left(q^{k}\right)^{j}\right)-\frac{\partial \rho}{\partial \bar{z}^{j}}\left(q^{k}\right)\left(z^{n}-\left(q^{k}\right)^{n}\right), & \text { for } 1 \leq j \leq n-1, \\
\left(z^{n}\right)^{*} & = & \sum_{j=1}^{n} \frac{\partial \rho}{\partial z^{j}}\left(q^{k}\right)\left(z^{j}-\left(q^{k}\right)^{j}\right) .
\end{aligned}\right.
$$


If $\delta_{k}:=\operatorname{dist}\left(p^{k}, \partial D\right)$ then $\alpha^{k}\left(p^{k}\right)=\left(0,-\delta_{k}\right)$ and $\alpha^{k}(D)=\left\{2 R e z^{n}+O\left(|z|^{2}\right)<0\right\}$ near the origin. Moreover, the sequence $\left(\alpha^{k}\right)_{*}(J)$ converges to $J$ as $k \rightarrow \infty$, since the sequence $\left(\alpha^{k}\right)_{k}$ converges to the identity map. Let $\left(L^{k}\right)_{k}$ be a sequence of linear automorphisms of $\mathbb{R}^{2 n}$ such that $\left(T^{k}:=L^{k} \circ \alpha^{k}\right)_{k}$ converges to the identity, and $D^{k}:=T^{k}(D)$ is defined near the origin by $D^{k}=\left\{\rho_{k}(z)=R e z^{n}+O\left(|z|^{2}\right)<0\right\}$. The sequence of almost complex structures $\left(J_{k}:=\left(T^{k}\right)_{*}(J)\right)_{k}$ converges to $J$ as $k \rightarrow \infty$ and $J_{k}(0)=J_{s t}$. Furthermore $\tilde{p}_{k}:=T^{k}\left(p^{k}\right)$ satisfies $\tilde{p}_{k}=\left(o\left(\delta_{k}\right), \delta_{k}^{\prime \prime}+i o\left(\delta_{k}\right)\right)$ with $\delta_{k}^{\prime \prime} \sim \delta_{k}$.

We proceed similarly on $D^{\prime}$. Denote by $s^{k}$ the projection of $f\left(p^{k}\right)$ onto $\partial D^{\prime}$ and define the transformation $\beta^{k}$ by

$$
\left\{\begin{aligned}
&\left(w^{j}\right)^{*}=\frac{\partial \rho^{\prime}}{\partial \bar{w}^{n}}\left(s^{k}\right)\left(w^{j}-\left(s^{k}\right)^{j}\right)-\frac{\partial \rho^{\prime}}{\partial \bar{w}^{j}}\left(s^{k}\right)\left(w^{n}-\left(s^{k}\right)^{n}\right), \quad \text { for } 1 \leq j \leq n-1, \\
&\left(w^{n}\right)^{*}= \\
& \sum_{j=1}^{n} \frac{\partial \rho^{\prime}}{\partial w^{j}}\left(s^{k}\right)\left(w^{j}-\left(s^{k}\right)^{j}\right) .
\end{aligned}\right.
$$

We define a sequence $\left(T^{\prime k}\right)_{k}$ of linear transformations converging to the identity and satisfying the following properties. The domain $\left(D^{k}\right)^{\prime}:=T^{\prime k}\left(D^{\prime}\right)$ is defined near the origin by $\left(D^{k}\right)^{\prime}=$ $\left\{\rho_{k}^{\prime}(w):=\operatorname{Rew}^{n}+O\left(|w|^{2}\right)<0\right\}$, and $\tilde{f}\left(p_{k}\right)=T^{\prime k}\left(f\left(p_{k}\right)\right)=\left(o\left(\varepsilon_{k}\right), \varepsilon_{k}^{\prime \prime}+i o\left(\varepsilon_{k}\right)\right)$ with $\varepsilon_{k}^{\prime \prime} \sim \varepsilon_{k}$, where $\varepsilon_{k}=\operatorname{dist}\left(f\left(p_{k}\right), \partial D^{\prime}\right)$. The sequence of almost complex structures $\left(J_{k}^{\prime}:=\left(T^{\prime k}\right)_{*}\left(J^{\prime}\right)\right)_{k}$ converges to $J^{\prime}$ as $k \rightarrow \infty$ and $J_{k}^{\prime}(0)=J_{s t}$.

Finally, the map $f^{k}:=T^{\prime k} \circ f \circ\left(T^{k}\right)^{-1}$ satisfies $f^{k}\left(\tilde{p}_{k}\right)=\tilde{f}\left(p_{k}\right)$ and is a $\left(J_{k}, J_{k}^{\prime}\right)$ biholomorphism between the domains $D^{k}$ and $\left(D^{\prime}\right)^{k}$.

Let $\phi_{k}:\left({ }^{\prime} z, z^{n}\right) \mapsto\left(\delta_{k}^{1 / 2 \prime} z, \delta_{k} z^{n}\right)$ and $\psi_{k}\left({ }^{\prime} w, w^{n}\right)=\left(\varepsilon_{k}^{1 / 2}{ }^{\prime} w, \varepsilon_{k} w^{n}\right)$ and set $\hat{f}^{k}=\left(\psi_{k}\right)^{-1} \circ f^{k} \circ$ $\phi_{k}$. The map $\hat{f}^{k}$ is $\left(\hat{J}_{k}, \hat{J}_{k}^{\prime}\right)$-biholomorphic, where $\hat{J}_{k}:=\left(\left(\phi_{k}\right)^{-1}\right)_{*}\left(J_{k}\right)$ and $\hat{J}_{k}^{\prime}:=\left(\psi_{k}^{-1}\right)_{*}\left(J_{k}^{\prime}\right)$. If $\hat{D}^{k}:=\phi_{k}^{-1}\left(D^{k}\right)$ and $\left(\hat{D}^{\prime}\right)^{k}:=\psi_{k}^{-1}\left(\left(D^{\prime}\right)^{k}\right)$ then $\hat{D}^{k}=\left\{z \in \phi_{k}^{-1}(U): \hat{\rho}_{k}(z)<0\right\}$ where

$$
\begin{aligned}
\hat{\rho}_{k}(z) & :=\delta_{k}^{-1} \rho\left(\phi_{k}(z)\right) \\
& =2 \operatorname{Re} z^{n}+\delta_{k}^{-1}\left[2 \operatorname{Re} K\left(\delta_{k}^{1 / 2 \prime} z, \delta_{k} z^{n}\right)+H\left(\delta_{k}^{1 / 2 \prime} z, \delta_{k} z^{n}\right)+o\left(\left|\left(\delta_{k}^{1 / 2 \prime} z, \delta_{k} z^{n}\right)\right|^{2}\right) .\right.
\end{aligned}
$$

and $\left(\hat{D}^{\prime}\right)^{k}=\left\{w \in \phi_{k}^{-1}(V): \hat{\rho}_{k}^{\prime}(z)<0\right\}$ where

$$
\begin{aligned}
\hat{\rho}_{k}^{\prime}(w) & :=\varepsilon_{k}^{-1} \rho^{\prime}\left(\psi_{k}(w)\right) \\
& =2 R e w^{n}+\varepsilon_{k}^{-1}\left[2 \operatorname{Re} K^{\prime}\left(\varepsilon_{k}^{1 / 2}{ }^{\prime} w, \varepsilon_{k} w^{n}\right)+H^{\prime}\left(\varepsilon_{k}^{1 / 2}{ }^{\prime} w, \varepsilon_{k} w^{n}\right)+o\left(\left|\left(\varepsilon_{k}^{1 / 2}{ }^{\prime} w, \varepsilon_{k} w^{n}\right)\right|^{2}\right) .\right.
\end{aligned}
$$

Since $U$ is a neighborhood of the origin, the pullbacks $\phi_{k}^{-1}(U)$ converge to $\mathbb{C}^{n}$ and the functions $\hat{\rho}_{k}$ converge to $\hat{\rho}(z)=2 \operatorname{Re} z^{n}+2 \operatorname{Re} K\left({ }^{\prime} z, 0\right)+H\left({ }^{\prime} z, 0\right)$ in the $\mathcal{C}^{2}$ norm on compact subsets of $\mathbb{C}^{n}$. Similarly, since $V$ is a neighborhood of the origin, the pullbacks $\psi_{k}^{-1}\left(U^{\prime}\right)$ converge to $\mathbb{C}^{n}$ and the functions $\hat{\rho}_{k}^{\prime}$ converge to $\hat{\rho}^{\prime}(w)=2 \operatorname{Re} w^{n}+2 \operatorname{Re} K^{\prime}\left({ }^{\prime} w, 0\right)+H^{\prime}\left({ }^{\prime} w, 0\right)$ in the $\mathcal{C}^{2}$ norm on compact subsets of $\mathbb{C}^{n}$. If $\Sigma:=\left\{z \in \mathbb{C}^{n}: \hat{\rho}(z)<0\right\}$ and $\Sigma^{\prime}:=\left\{w \in \mathbb{C}^{n}\right.$ : $\left.\hat{\rho}^{\prime}(w)<0\right\}$ the sequence of points $\hat{p}_{k}=\phi_{k}^{-1}\left(\tilde{p}_{k}\right) \in \hat{D}^{k}$ converges to the point $(0,-1) \in \Sigma$ and the sequence of points $\hat{f}\left(p_{k}\right)=\psi_{k}^{-1}\left(\tilde{f}\left(p_{k}\right)\right) \in \hat{D}^{\prime{ }^{k}}$ converges to $(0,-1) \in \Sigma^{\prime}$. Finally $\hat{f}^{k}\left(\hat{p}_{k}\right)=\hat{f}\left(p_{k}\right)$.

The limit behaviour of the dilated objects is given by the following proposition (see Figure 6).

Proposition 4.3.3. $(i)$ The sequences $\left(\hat{J}_{k}\right)$ and $\left(\hat{J}_{k}^{\prime}\right)$ of almost complex structures converge to model structures $J_{0}$ and $J_{0}^{\prime}$ uniformly (with all partial derivatives of any order) on compact subsets of $\mathbb{C}^{n}$. 
(ii) $\left(\Sigma, J_{0}\right)$ and $\left(\Sigma^{\prime}, J_{0}^{\prime}\right)$ are model domains.

(iii) The sequence $\left(\hat{f}^{k}\right)$ (together with all derivatives) is a relatively compact family (with respect to the compact open topology) on $\Sigma$; every cluster point $\hat{f}$ is a $\left(J_{0}, J_{0}^{\prime}\right)$-biholomorphism between $\Sigma$ and $\Sigma^{\prime}$, satisfying $\hat{f}(0,-1)=(0,-1)$ and $\hat{f}^{n}\left({ }^{\prime} 0, z^{n}\right)=z^{n}$ on $\Sigma$.

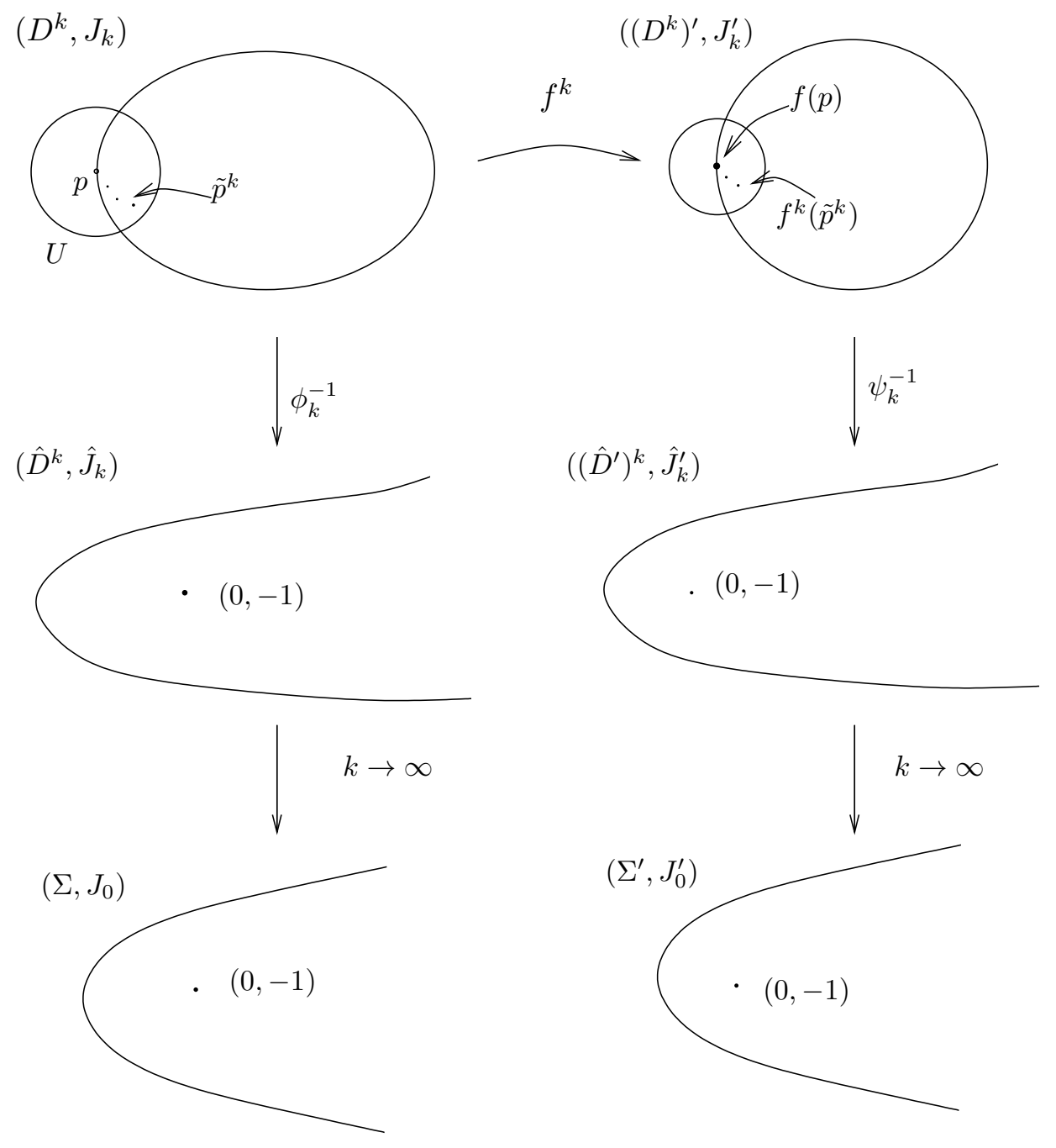

Figure 6

Proof of Proposition 4.3.3. We start with the proof of $(i)$. We focus on structures $\hat{J}_{k}$. Consider $J=J_{s t}+L(z)+O\left(|z|^{2}\right)$ as a matrix valued function, where $L$ is a real linear matrix. The Taylor expansion of $J_{k}$ at the origin is given by $J_{k}=J_{s t}+L^{k}(z)+O\left(|z|^{2}\right)$ on $U$, uniformly with respect to $k$. Here $L^{k}$ is a real linear matrix converging to $L$ at infinity. Write $\hat{J}_{k}=J_{s t}+\hat{L}^{k}+O\left(\delta_{k}\right)$. If $L^{k}=\left(L_{j, l}^{k}\right)_{j, l}$ then $\hat{L}_{j, l}^{k}=L_{j, l}^{k}\left(\phi_{k}(z)\right)$ for $1 \leq j \leq n-1,1 \leq l \leq n$, $\hat{L}_{n, l}^{k}=\delta_{k}^{-1 / 2} L_{n, l}^{k}\left(\phi_{k}(z)\right)$ for $1 \leq l \leq n-1$ and $\hat{L}_{n, n}^{k}=L_{n, n}^{k}\left(\phi_{k}(z)\right)$. This gives the conclusion. 
Proof of $(i i)$. We focus on $\left(\Sigma, J_{0}\right)$. By the invariance of the Levi form we have $\mathcal{L}^{J_{k}}\left(\rho_{k}\right)(0)\left(\phi_{k}(v)\right)=\mathcal{L}^{\hat{J}_{k}}\left(\rho_{k} \circ \phi_{k}\right)(0)(v)$. Write $J_{0}=J_{s t}+L^{\infty}$. Since $\rho_{k}$ is strictly $J_{k^{-}}$ plurisubharmonic uniformly with respect to $k$ ( $\rho_{k}$ converges to $\rho$ and $J_{k}$ converges to $J$ ), multiplying by $\delta_{k}^{-1}$ and passing to the limit at the right side as $k \rightarrow \infty$, we obtain that $\mathcal{L}^{J_{0}}(\hat{\rho})(0)(v) \geq 0$ for any $v$. Now let $v=\left(v^{\prime}, 0\right) \in T_{0}(\partial \Sigma)$. Then $\phi_{k}(v)=\delta_{k}^{1 / 2} v$ and so $\mathcal{L}_{k}^{J}(\rho)(0)(v)=\mathcal{L}^{\hat{J}_{k}}\left(\rho_{k}\right)(0)(v)$. Passing to the limit as $k$ tends to infinity, we obtain that $\mathcal{L}^{J_{0}}(\hat{\rho})(0)(v)>0$ for any $v=\left(v^{\prime}, 0\right)$ with $v^{\prime} \neq 0$.

Proof of (iii). The proof of the existence and of the biholomorphicity of $\hat{f}$ is the same as in dimension two. We prove the identity on $\hat{f}^{n}$. Let $t$ be a real positive number. Then we have :

Lemma 4.3.4. $\lim _{t \rightarrow \infty} \hat{\rho}^{\prime}\left(\hat{f}^{\prime}(0,-t)\right)=\infty$.

Proof of Lemma 4.3.4. According to the boundary distance property (4.2) we have

$$
\left|\rho^{\prime}\left(f \circ\left(T^{k}\right)^{-1} \circ \phi_{k}\right)\left({ }^{\prime} 0,-t\right)\right| \geq C \operatorname{dist}\left(T_{k}^{-1}\left({ }^{\prime} 0,-\delta_{k} t\right)\right) .
$$

Then

$$
\left|\hat{\rho}_{k}^{\prime}\left(\hat{f}^{k}\left({ }^{\prime} 0,-t\right)\right)\right| \geq C \varepsilon_{k}^{-1} \delta_{k} t .
$$

Since $\hat{\rho}_{k}^{\prime}$ converges to $\hat{\rho}^{\prime}$ uniformly on compact subsets of $\Sigma^{\prime}$ and $\varepsilon_{k} \simeq \delta_{k}$ (by the boundary distance property (4.2) ) we obtain :

$$
\left|\hat{\rho}^{\prime}\left(\hat{f}\left({ }^{\prime} 0,-t\right)\right)\right| \geq C t .
$$

This proves Lemma 4.3.4.

We turn back to the proof of part (iii) of Proposition 4.3.3. Assume first that $J$ (and similarly $J^{\prime}$ ) are not integrable (see Proposition 1.3.10). Consider a $J$-complex hypersurface $A \times \mathbb{C}$ in $\mathbb{C}^{n}$ where $A$ is a $J_{s t}$ complex hypersurface in $\mathbb{C}^{n-1}$. Since $f\left((A \times \mathbb{C}) \cap \mathbb{H}_{P_{1}}\right)=$ $\left(A^{\prime} \times \mathbb{C}\right) \cap \mathbb{H}_{P_{2}}$ where $A^{\prime}$ is a $J_{s t}$ complex hypersurface in $\mathbb{C}^{n-1}$, it follows that the restriction of $\hat{f}^{n}$ to $\left\{{ }^{\prime} z={ }^{\prime} 0, \operatorname{Re}\left(z^{n}\right)<0\right\}$ is a $J_{s t}$ automorphism of $\left\{{ }^{\prime} z={ }^{\prime} 0, \operatorname{Re}\left(z^{n}\right)<0\right\}$. Let $\phi: \zeta \mapsto(\zeta-1) /(\zeta+1)$. The function $\hat{g}:=\phi^{-1} \circ \hat{f}^{n} \circ \phi$ is a $J_{s t}$ automorphism of the unit disc in $\mathbb{C}$. In view of Lemma 4.3 .4 this satisfies $\hat{g}(0)=0$ and $\hat{g}(1)=1$. Hence $\hat{g} \equiv i d$ and $\hat{f}^{n}\left({ }^{\prime} 0, z^{n}\right)=z^{n}$ on $\Sigma$.

Assume now that $J$ and $J^{\prime}$ are integrable. Let $F$ (resp. $F^{\prime}$ ) be the diffeomorphism from $\Sigma$ to $\mathbb{H}_{P}$ (resp. from $\Sigma$ to $\mathbb{H}_{P^{\prime}}$ ) given in the proof of Proposition 1.3.10. The diffeomorphism $g:=F^{\prime} \circ f \circ F^{-1}$ is a $J_{s t^{-}}$-biholomorphism from $\mathbb{H}_{P}$ to $\mathbb{H}_{P^{\prime}}$ satisfying $g\left({ }^{\prime} 0,-1\right)=\left({ }^{\prime} 0,-1\right)$. Since $(\Sigma, J)$ and $\left(\Sigma^{\prime}, J^{\prime}\right)$ are model domains, the domains $\mathbb{H}_{P}$ and $\mathbb{H}_{P^{\prime}}$ are strictly $J_{s t^{-}}$ pseudoconvex. In particular, since $P$ and $P^{\prime}$ are homogeneous of degree two, there are linear complex maps $L, L^{\prime}$ in $\mathbb{C}^{n-1}$ such that the map $G$ (resp. $\left.G^{\prime}\right)$ defined by $G\left({ }^{\prime} z, z_{n}\right)=\left(L\left({ }^{\prime} z\right), z_{n}\right)$ (resp. $\left.G^{\prime}\left({ }^{\prime} z, z_{n}\right)=\left(L^{\prime}\left({ }^{\prime} z\right), z_{n}\right)\right)$ is a biholomorphism from $\mathbb{H}_{P}$ (resp. $\left.\mathbb{H}_{P^{\prime}}\right)$ to $\mathbb{H}$. The map $G^{\prime} \circ g \circ G^{-1}$ is an automorphism of $\mathbb{H}$ satisfying $G^{\prime} \circ g \circ G^{-1}\left({ }^{\prime} 0,-1\right)=\left({ }^{\prime} 0,-1\right)$. Let $\Phi$ be the $J_{s t}$ biholomorphism from $\mathbb{H}$ to the unit ball $\mathbb{B}_{n}$ of $\mathbb{C}^{n}$ defined by $\Phi\left({ }^{\prime} z, z^{n}\right)=\left(\sqrt{2}^{\prime} z / 1-\right.$ $\left.z^{n},\left(1+z^{n}\right) /\left(1-z^{n}\right)\right)$. Let $\hat{g}:=\Phi^{-1} \circ g \circ \Phi$. In view of lemma 4.2 this satisfies $\hat{g}(0)=0$ and $\hat{g}\left({ }^{\prime} 0,1\right)=\left({ }^{\prime} 0,1\right)$. Hence $\hat{g}^{n} \equiv i d$ and $\hat{f}^{n}\left({ }^{\prime} z, z^{n}\right)=z^{n}$ for every $z$ in $\Sigma$. 
According to part $(\mathrm{ii})$ of Proposition 4.3 .3 and restricting $U$ if necessary, one may view $D \cap U$ as a strictly $J_{0}$-pseudoconvex domain in $\mathbb{C}^{n}$ and $J$ as a small deformation of $J_{0}$ in a neighborhood of $\bar{D} \cap U$. The same holds for $D^{\prime} \cap V$.

For $p \in \partial D$ and $z \in D$ let $X_{p}(z)$ and $X_{f(p)}^{\prime}(f(z))$ be the basis of $(1,0)$ vector fields defined above. The elements of the matrix of $d f_{z}$ in the bases $X_{p}(z)$ and $X_{f(p)}^{\prime}(f(z))$ are denoted by $A_{j s}(p, z)$. According to Proposition 4.3.2 the function $A_{n, n}(p, \cdot)$ is upper bounded on $D$.

Proposition 4.3.5. We have:

(a) Every cluster point of the function $z \mapsto A_{n, n}(p, z)$ is real when $z$ tends to $p \in \partial D$.

(b) For $z \in D$, let $p \in \partial D$ such that $|z-p|=\operatorname{dist}(z, \partial D)$. There exists a constant $A$, independent of $z \in D$, such that $\left|A_{n, n}(p, z)\right| \geq A$.

Proof of Proposition 4.3.5. (a) Suppose that there exists a sequence of points $\left(p^{k}\right)$ converging to a boundary point $p$ such that $A_{n, n}(p, \cdot)$ tends to a complex number $a$. Applying the above scaling construction, we obtain a sequence of maps $\left(\hat{f}^{k}\right)_{k}$. For $k \geq 0$ consider the dilated vector fields

$$
Y_{k}^{j}:=\delta_{k}^{1 / 2}\left(\left(\phi_{k}^{-1}\right) \circ T^{k}\right)\left(X^{j}\left(p^{k}\right)\right)
$$

for $j=1, \ldots, n-1$, and

$$
Y_{k}^{n}:=\delta_{k}\left(\left(\phi_{k}^{-1}\right) \circ T^{k}\right)\left(X_{n}\left(p^{k}\right)\right)
$$

Similarly we define

$$
Y_{k}^{\prime j}:=\varepsilon_{k}^{-1 / 2}\left(\left(\psi_{k}^{-1}\right) \circ T^{\prime k}\right)\left(X^{\prime j}\left(f\left(p^{k}\right)\right)\right)
$$

for $j=1, \ldots, n-1$, and

$$
Y_{k}^{\prime n}:=\varepsilon_{k}^{-1}\left(\left(\psi_{k}^{-1}\right) \circ T^{\prime k}\right)\left(X_{n}^{\prime}\left(f\left(p^{k}\right)\right)\right)
$$

For every $k$, the $n$-tuple $Y^{k}:=\left(Y_{k}^{1}, \ldots, Y_{k}^{n}\right)$ is a basis of $(1,0)$ vector fields for the dilated structure $\hat{J}^{k}$. In view of Proposition 4.3 .3 the sequence $\left(Y^{k}\right)_{k}$ converges to a basis of $(1,0)$ vector fields of $\mathbb{C}^{n}$ (with respect to $J_{0}$ ) as $k$ tends to $\infty$. Similarly, the $n$-tuple $Y^{\prime k}:=$ $\left(Y_{k}^{\prime 1}, \ldots, Y_{k}^{\prime n}\right)$ is a basis of $(1,0)$ vector fields for the dilated structure $\hat{J}^{\prime k}$ and $\left(Y^{\prime k}\right)_{k}$ converges to a basis of $(1,0)$ vector fields of $\mathbb{C}^{n}$ (with respect to $J_{0}^{\prime}$ ) as $k$ tends to $\infty$. In particular the last components $Y_{k}^{n}$ and $Y_{k}^{\prime n}$ converge to the $(1,0)$ vector field $\partial / \partial z^{n}$. Denote by $\hat{A}_{j s}^{k}$ the elements of the matrix of $d \hat{f}^{k}(0,-1)$. Then $A_{n, n}^{k}$ converges to $\left(\partial \hat{f}^{n} / \partial z^{n}\right)(0,-1)=1$, according to Proposition 4.3.3. On the other hand, $A_{n, n}^{k}=\varepsilon_{k}^{-1} \delta_{k} A_{n, n}$ converges to $a$ by the boundary distance preserving property (4.2). This gives the statement.

(b) Suppose that there is a sequence of points $\left(p^{k}\right)$ converging to the boundary such that $A_{n, n}$ tends to 0 . Repeating precisely the argument of $(\mathrm{a})$, we obtain that $\left(\partial \hat{f}^{n} / \partial z^{n}\right)(0,-1)=$ 0 ; this contradicts part (iii) of Proposition 4.3.3.

Proposition 4.3.6. The cluster set of the cotangent lift $f^{*}$ on $\Sigma(\partial D)$ is contained in $\Sigma\left(\partial D^{\prime}\right)$.

Proof of Proposition 4.3.6.

Step one. We first reduce the problem to the following local situation. Let $D$ and $D^{\prime}$ be domains in $\mathbb{C}^{n}, \Gamma$ and $\Gamma^{\prime}$ be open $\mathcal{C}^{\infty}$-smooth pieces of their boundaries, containing the origin. We assume that an almost complex structure $J$ is defined and $\mathcal{C}^{\infty}$-smooth in a neighborhood 
of the closure $\bar{D}, J(0)=J_{s t}$. Similarly, we assume that $J^{\prime}(0)=J_{s t}$. The hypersurface $\Gamma$ (resp. $\Gamma^{\prime}$ ) is supposed to be strictly $J$-pseudoconvex (resp. strictly $J^{\prime}$-pseudoconvex). Finally, we assume that $f: D \rightarrow D^{\prime}$ is a $\left(J, J^{\prime}\right)$-biholomorphic map. It follows from the estimates of the Kobayashi-Royden infinitesimal pseudometric given in [33] that $f$ extends as a 1/2-Hölder homeomorphism between $D \cup \Gamma$ and $D^{\prime} \cup \Gamma^{\prime}$, such that $f(\Gamma)=\Gamma^{\prime}$ and $f(0)=0$. Finally $\Gamma$ is defined in a neighborhood of the origin by the equation $\rho(z)=0$ where $\rho(z)=2 \operatorname{Re} z^{n}+2 \operatorname{Re} K(z)+H(z)+o\left(|z|^{2}\right)$ and $K(z)=\sum K_{\mu \nu} z^{\mu \nu}, H(z)=\sum h_{\mu \nu} z^{\mu} \bar{z}^{\nu}$, $k_{\mu \nu}=k_{\nu \mu}, h_{\mu \nu}=\bar{h}_{\nu \mu}$. As we noticed at the end of Section 3 the hypersurface $\Gamma$ is strictly $\hat{J}$-pseudoconvex at the origin. The hypersurface $\Gamma^{\prime}$ admits a similar local representation. In what follows we assume that we are in this setting.

Let $\Sigma:=\left\{z \in \mathbb{C}^{n}: 2 \operatorname{Re} z^{n}+2 \operatorname{Re} K\left({ }^{\prime} z, 0\right)+H\left({ }^{\prime} z, 0\right)<0\right\}, \quad \Sigma^{\prime}:=\left\{z \in \mathbb{C}^{n}:\right.$ $\left.2 R e z^{n}+2 \operatorname{Re} K^{\prime}\left({ }^{\prime} z, 0\right)+H^{\prime}\left({ }^{\prime} z, 0\right)<0\right\}$. If $\left(p^{k}\right)$ is a sequence of points in $D$ converging to 0 , then according to Proposition 4.3.3, the scaling procedure associates with the pair $\left(f,\left(p^{k}\right)_{k}\right)$ two linear almost complex structures $J_{0}$ and $J_{0}^{\prime}$, both defined on $\mathbb{C}^{n}$, and a $\left(J_{0}, J_{0}^{\prime}\right)$ biholomorphism $\hat{f}$ between $\Sigma$ and $\Sigma^{\prime}$. Moreover $\left(\Sigma, J_{0}\right)$ and $\left(\Sigma^{\prime}, J_{0}^{\prime}\right)$ are model domains. To prove that the cluster set of the cotangent lift of $f$ at a point in $N(\Gamma)$ is contained in $N\left(\Gamma^{\prime}\right)$, it is sufficient to prove that $\left(\partial \hat{f}^{n} / \partial z^{n}\right)\left({ }^{\prime} 0,-1\right) \in \mathbb{R} \backslash\{0\}$.

Step two. The proof of Proposition 4.3.6 is given by the following Lemma.

Lemma 4.3.7. Let $K$ be a compact subset of the totally real part of the conormal bundle $\Sigma_{J}(\partial D)$. Then the cluster set of the cotangent lift $f^{*}$ of $f$ on the conormal bundle $\Sigma(\partial D)$, when $(z, L)$ tends to $\Sigma_{J}(\partial D)$ along the wedge $W_{U}$, is relatively compactly contained in the totally real part of $\Sigma\left(\partial D^{\prime}\right)$.

We recall that the totally real part of $\Sigma\left(\partial D^{\prime}\right)$ is the complement of the zero section in $\Sigma\left(\partial D^{\prime}\right)$.

Proof of Lemma 4.3.7. Let $\left(z^{k}, L^{k}\right)$ be a sequence in $W_{U}$ converging to $\left(0, \partial_{J} \rho(0)\right)=\left(0, d z^{n}\right)$. We shall prove that the sequence of linear forms $Q^{k}:={ }^{t} d f^{-1}\left(w^{k}\right) L^{k}$, where $w^{k}=f\left(z^{k}\right)$, converges to a linear form which up to a real factor (in view of Part (a) of Proposition 4.3.5) coincides with $\partial_{J} \rho(0)=d z^{n}$ (we recall that ${ }^{t}$ denotes the transposed map). It is sufficient to prove that the $(n-1)$ first component of $Q^{k}$ with respect to the dual basis $\left(\omega_{1}, \ldots, \omega_{n}\right)$ of $X$ converge to 0 and the last one is bounded below from the origin as $k$ goes to infinity. The map $X$ being of class $\mathcal{C}^{1}$ we can replace $X(0)$ by $X\left(w^{k}\right)$. Since $\left(z^{k}, L^{k}\right) \in W_{U}$, we have $L^{k}=\omega_{n}\left(z^{k}\right)+O\left(\delta_{k}\right)$, where $\delta_{k}$ is the distance from $z^{k}$ to the boundary. Since $\left\|\left|d f_{w^{k}}^{-1}\right|\right\|=0\left(\delta_{k}^{-1 / 2}\right)$, we have $Q^{k}={ }^{t} d f_{w^{k}}^{-1}\left(\omega_{n}\left(z^{k}\right)\right)+O\left(\delta_{k}^{1 / 2}\right)$. By Proposition 4.3.3, the components of ${ }^{t} d f_{w^{k}}^{-1}\left(\omega_{n}\left(z^{k}\right)\right)$ with respect to the basis $\left(\omega_{1}\left(z^{k}\right), \ldots, \omega_{n}\left(z^{k}\right)\right)$ are the elements of the last line of the matrix $d f_{w^{k}}^{-1}$ with respect to the basis $X^{\prime}\left(w^{k}\right)$ and $X\left(z^{k}\right)$. So its $(n-1)$ first components are $0\left(\delta_{k}^{1 / 2}\right)$ and converge to 0 as $k$ tends to infinity. Finally the component $A_{n, n}^{k}$ is bounded below from the origin by Part (b) of Proposition 4.3.5.

\subsubsection{Compactness principle. In this section we prove the following}

Theorem 4.3.8. Let $(M, J)$ be an almost complex manifold, not equivalent to a model domain. Let $D=\{r<0\}$ be a relatively compact domain in a smooth manifold $N$ and let $\left(f^{\nu}\right)_{\nu}$ be a sequence of diffeomorphisms from $M$ to $D$. Assume that 
(i) the sequence $\left(J_{\nu}:=f_{*}^{\nu}(J)\right)_{\nu}$ extends smoothly up to $\bar{D}$ and is compact in the $C^{2}$ convergence on $\bar{D}$,

(ii) the Levi forms of $\partial D, \mathcal{L}^{J_{\nu}}(\partial D)$ are uniformly bounded from below (with respect to $\nu$ ) by a positive constant.

Then the sequence $\left(f^{\nu}\right)_{\nu}$ is compact in the compact-open topology on $M$.

We proceed by contradiction. Assume that there is a compact $K_{0}$ in $M$, points $p^{\nu} \in M$ and a point $q \in \partial D$ such that $\lim _{\nu \rightarrow \infty} f^{\nu}\left(p^{\nu}\right)=q$.

Lemma 4.3.9. For every relatively compact neighborhood $V$ of $q$ there is $\nu_{0}$ such that for $\nu \geq \nu_{0}$ we have : $\lim _{x \rightarrow q}$ in $f_{q^{\prime} \in D \cap \partial V} d_{\left(D, J_{\nu}\right)}^{K}=\infty$.

Proof of Lemma 4.3.9. Restricting $U$ if necessary, we may assume that the function $\rho+C \rho^{2}$ is a strictly $J_{\nu}$-plurisubharmonic function in a neighborhood of $\bar{D} \cap U$, for sufficiently large $\nu$. Moreover, using Proposition B, we can focus on $K_{D \cap U}$. Smoothing $D \cap U$, we may assume that the hypothesis of Proposition A are satisfied on $D \cap U$, uniformly for sufficiently large $\nu$. In particular, the inequality (3.4) is satisfied on $D \cap U$, with a positive constant $c$ independent of $\nu$. The result follows by a direct integration of this inequality.

The following Lemma is a corollary of Lemma 4.3.9.

Lemma 4.3.10. For every $K \subset \subset M$ we have : $\lim _{\nu \rightarrow \infty} f^{\nu}(K)=q$.

Proof of Lemma 4.3.10, Let $K \subset \subset M$ be such that $x^{0} \in K$. Since the function $x \mapsto d_{D}^{K}\left(x^{0}, x\right)$ is bounded from above by a constant $C$ on $K$, it follows from the decreasing property of the Kobayashi pseudodistance that

$$
d_{\left(D, J_{\nu}\right)}^{K}\left(f^{\nu}\left(x^{0}\right), f^{\nu}(x)\right) \leq C
$$

for every $\nu$ and every $x \in K$. It follows from Lemma 4.3.9 that for every $V \subset \subset U$, containing $p$, we have :

$$
\lim _{\nu \rightarrow \infty} d_{\left(D, J_{\nu}\right)}^{K}\left(f^{\nu}\left(x^{0}\right), D \cap \partial V\right)=+\infty
$$

Then from conditions (4.3) and (4.4) we deduce that $f^{\nu}(K) \subset V$ for every sufficiently large $\nu$. This gives the statement.

Fix now a point $p \in M$ and denote by $p^{\nu}$ the point $f^{\nu}(p)$. We may assume that the sequence $\left(J_{\nu}:=f_{*}^{\nu}(J)\right)_{\nu}$ converges to an almost complex structure $J^{\prime}$ on $\bar{D}$ and according to Lemma 4.3.10 we may assume that $\lim _{\nu \rightarrow \infty} p^{\nu}=q$. We apply Subsection 4.3 to the domain $D$ and the sequence $\left(q^{\nu}\right)_{\nu}$. We denote by $T^{\nu}$ the linear transformation $T^{\nu}:=M^{\nu} \circ L^{\nu} \circ \alpha^{\nu}$, as in Subsection 4.3, and we consider $D^{\nu}:=T^{\nu}(D)$, and $J^{\nu}:=T_{*}^{\nu}\left(J_{\nu}\right)$. If $\phi_{\nu}$ is the nonisotropic dilation $\phi_{\nu}:\left({ }^{\prime} z, z^{n}\right) \mapsto\left(\delta_{\nu}^{1 / 2}{ }^{\prime} z, \delta_{\nu} z^{n}\right)$ then we set $\hat{f}^{\nu}:=\phi_{\nu}^{-1} \circ T^{\nu} \circ f$ and $\hat{J}^{\nu}:=\left(\phi_{\nu}^{-1}\right)_{*}\left(J^{\nu}\right)$. We also consider $\hat{\rho}_{\nu}:=\delta_{\nu}^{-1} \circ \rho \circ \phi_{\nu}$ and $\hat{D}^{\nu}:=\left\{\hat{\rho}_{\nu}<0\right\}$. As proved in Subsection 4.3, the sequence $\left(\hat{D}^{\nu}\right)_{\nu}$ converges, in the local Hausdorff convergence, to a domain $\Sigma:=\left\{z \in C^{n}\right.$ : $\left.\hat{\rho}(z):=2 \operatorname{Re} z^{n}+2 \operatorname{Re} K\left({ }^{\prime} z, 0\right)+H\left({ }^{\prime} z, 0\right)<0\right\}$, where $K$ and $H$ are homogeneous of degree two. According to Proposition 4.3 .3 we have :

(i) The sequence $\left(\hat{J}^{\nu}\right)$ converges to a model almost complex structure $J_{0}$, uniformly (with all partial derivatives of any order) on compact subsets of $\mathbb{C}^{n}$, 
(ii) $\left(\Sigma, J_{0}\right)$ is a model domain,

(iii) the sequence $\left(\hat{f}^{\nu}\right)_{\nu}$ converges to a $\left(J, J_{0}\right)$ holomorphic map $F$ from $M$ to $\Sigma$.

To prove Theorem 4.3.8, it remains to prove that $F$ is a diffeomorphism from $M$ to $\Sigma$. We first notice that according to condition $(i i)$ of Theorem 4.3.8 and Lemma 4.3.9, the domain $D$ is complete $J_{\nu}$-hyperbolic. In particular, since $f^{\nu}$ is a $\left(J, J_{\nu}\right)$ biholomorphism from $M$ to $D$, the manifold $M$ is complete $J$-hyperbolic. Consequently, for every compact subset $L$ of $M$, there is a positive constant $C$ such that for every $z \in L$ and every $v \in T_{z} M$ we have $K_{(M, J)}(z, v) \geq C\|v\|$. Consider the map $\hat{g}^{\nu}:=\left(\hat{f}^{\nu}\right)^{-1}$. This is a $\left(\hat{J}^{\nu}, J\right)$ biholomorphism from $\hat{D}^{\nu}$ to $M$. Let $K$ be a compact set in $\Sigma$. We may consider $\hat{g}^{\nu}(K)$ for sufficiently large $\nu$. By the decreasing property of the Kobayashi distance, there is a compact subset $L$ in $M$ such that $\hat{g}^{\nu}(K) \subset L$ for sufficiently large $\nu$. Then for every $w \in K$ and for every $v \in T_{w} \Sigma$ we obtain, by the decreasing of the Kobayashi-Royden infinitesimal pseudometric :

$$
\left\|d f^{\nu}(w)(v)\right\| \leq(1 / C)\|v\|,
$$

uniformly for sufficiently large $\nu$. According to Ascoli Theorem, we may extract from $\left(\hat{g}^{\nu}\right)_{\nu}$ a subsequence, converging to a map $G$ from $\Sigma$ to $M$. Finally, on any compact subset $K$ of $M$, by the equality $\hat{g}^{\nu} \circ \hat{f}^{\nu}=i d$ we obtain $F \circ G=i d$. This gives the result.

As a corollary of Theorem 4.3.8 we obtain the following almost complex version of the Wong-Rosay Theorem in real dimension four :

Corollary 4.3.11. Let $(M, J)$ (resp. $\left(M^{\prime}, J^{\prime}\right)$ ) be an almost complex manifold of real dimension four. Let $D$ (resp. $\left.D^{\prime}\right)$ be a relatively compact domain in $M$ (resp. N). Consider a sequence $\left(f^{\nu}\right)_{\nu}$ of diffeomorphisms from $D$ to $D^{\prime}$ such that the sequence $\left(J_{\nu}:=f_{*}^{\nu}(J)\right)_{\nu}$ extends to $\bar{D}^{\prime}$ and converges to $J^{\prime}$ in the $C^{2}$ convergence on $\bar{D}^{\prime}$.

Assume that there is a point $p \in D$ and a point $q \in \partial D^{\prime}$ such that $\lim _{\nu \rightarrow \infty} f^{\nu}(p)=q$ and such that $D^{\prime}$ is strictly $J^{\prime}$-pseudoconvex at $q$. Then there is a $\left(J, J_{\text {st }}\right)$-biholomorphism from $M$ to the unit ball $\mathbb{B}^{2}$ in $\mathbb{C}^{2}$.

Proof of Corollary 4.3.11. The proof of Corollary 4.3.11 follows exactly the same lines as the proof of Theorem 4.3.8.

\section{Elliptic REGUlARITY ON ALMOST COMPLEX MANIFOLDS With BOUNDARY}

This section is devoted to one of the main technical steps of our construction. We prove that a pseudoholomorphic disc attached (in the sense of the cluster set) to a smooth totally real submanifold in an almost complex manifold, extends smoothly up to the boundary. In the case of the integrable structure, various versions of this statement have been obtained by several authors. In the almost complex case, similar assertions have been established by H.Hofer [42, J.-C.Sikorav [73], S.Ivashkovich-V.Shevchishin [45], E.Chirka [18], D.McDuffD.Salamon [59] under stronger assumptions on the initial boundary regularity of the disc (at least the continuity is required). Our proof consists of two steps. First, we show that a disc extends as a 1/2-Hölder continuous map up to the boundary. The proof is based on special estimates of the Kobayashi-Royden metric in "Grauert tube" type domains. The second step is the reflection principle adapted to the almost complex category; here we follow the construction of E.Chirka [18]. 
5.1. Reflection principle and regularity of analytic discs. We prove the following :

Theorem 5.1.1. Let $N$ be a smooth $\mathcal{C}^{\infty}$ totally real submanifold in $(M, J)$ and let $\varphi: \Delta^{+} \rightarrow$ $M$ be J-holomorphic, where $\Delta^{+}:=\{\zeta \in \Delta: \operatorname{Im}(\zeta)>0\}$. Assume that the cluster set of $\varphi$ on the real interval ] - 1, 1 [is contained in $N$. Then $\varphi$ is of class $\mathcal{C}^{\infty}$ on $\left.\Delta^{+} \cup\right]-1,1[$.

In case $N$ has a weaker regularity then the exact regularity of $\varphi$, related to that of $N$, can be derived directly from the following proof of Theorem 5.1.1.

Proof of Theorem 5.1.1. Step one. It follows by Theorem 3.3.3 that $\varphi$ extends as a Hölder 1/2-continuous map on $\left.\Delta^{+} \cup\right]-1,1[$.

Step two : The disc $\varphi$ is of class $\mathcal{C}^{1+1 / 2}$. The following construction of the reflection principle for pseudoholomorphic discs is due to Chirka [18]. For reader's convenience we give the details. Let $a \in]-1,1\left[\right.$. Our consideration being local at $a$, we may assume that $N=\mathbb{R}^{n} \subset$ $\mathbb{C}^{n}, a=0$ and $J$ is a smooth almost complex structure defined in the unit ball $\mathbb{B}_{n}$ in $\mathbb{C}^{n}$.

After a complex linear change of coordinates we may assume that $J=J_{s t}+O(|z|)$ and $N$ is given by $x+i h(x)$ where $x \in \mathbb{R}^{n}$ and $d h(0)=0$. If $\Phi$ is the local diffeomorphism $x \mapsto x, y \mapsto$ $y-h(x)$ then $\Phi(N)=\mathbb{R}^{n}$ and the direct image of $J$ by $\Phi$, still denoted by $J$, keeps the form $J_{s t}+O(|z|)$. Then $J$ has a basis of (1,0)-forms given in the coordinates $z$ by $d z^{j}+\sum_{k} a_{j k} d \bar{z}^{k}$; using the matrix notation we write it in the form $\omega=d z+A(z) d \bar{z}$ where the matrix function $A(z)$ vanishes at the origin. Writing $\omega=(I+A) d x+i(I-A) d y$ where $I$ denotes the identity matrix, we can take as a basis of $(1,0)$ forms : $\omega^{\prime}=d x+i(I+A)^{-1}(I-A) d y=d x+i B d y$. Here the matrix function $B$ satisfies $B(0)=I$. Since $B$ is smooth, its restriction $B_{\mid \mathbb{R}^{n}}$ on $\mathbb{R}^{n}$ admits a smooth extension $\hat{B}$ on the unit ball such that $\hat{B}-B_{\mid \mathbb{R}^{n}}=O\left(|y|^{k}\right)$ for any positive integer $k$. Consider the diffeomorphism $z^{*}=x+i \hat{B}(z) y$. In the $z^{*}$-coordinates the submanifold $N$ still coincides with $\mathbb{R}^{n}$ and $\omega^{\prime}=d x+i B d y=d z^{*}+i(B-\hat{B}) d y-i(d \hat{B}) y=d z^{*}+\alpha$, where the coefficients of the form $\alpha$ vanish up to the first order on $\mathbb{R}^{n}$. Therefore there is a basis of $(1,0)$-forms (with respect to the image of $J$ under the coordinate diffeomorphism $z \mapsto z^{*}$ ) of the form $d z^{*}+A\left(z^{*}\right) d \bar{z}^{*}$, where $A$ vanishes to first order on $\mathbb{R}^{n}$ and $\|A\|_{\mathcal{C}^{1}\left(\overline{\mathbb{B}}_{n}\right)}<<1$.

Consider the continuous map $\psi$ defined on $\Delta$ by

$$
\left\{\begin{array}{cc}
\psi=\varphi & \text { on } \Delta^{+} \\
\psi(\zeta)=\overline{\varphi(\bar{\zeta})} & \text { for } \zeta \in \Delta^{-}:=\{\zeta \in \Delta / \operatorname{Im}(\zeta)<0\} .
\end{array}\right.
$$

Since the map $\varphi$ satisfies

$$
\bar{\partial} \varphi+A(\varphi) \overline{\partial \varphi}=0
$$

on $\Delta^{+}$, the map $\psi$ satisfies the equation

$$
\bar{\partial} \psi(\zeta)+\overline{A(\varphi(\bar{\zeta}))} \overline{\partial \psi(\zeta)}=0
$$

for $\zeta \in \Delta^{-}$.

Hence $\psi$ is a solution on $\Delta$ of the elliptic equation

$$
\bar{\partial} \psi+\lambda(\cdot) \overline{\partial \psi}=0
$$


where $\lambda$ is defined by $\lambda(\zeta)=A(\varphi(\zeta))$ for $\left.\zeta \in \Delta^{+} \cup\right]-1,1\left[\right.$ and $\lambda(\zeta)=\overline{A(\varphi(\bar{\zeta}))}$ for $\zeta \in \Delta^{-}$. According to Step one, the map $\lambda$ is Hölder $1 / 2$ continuous on $\Delta$ and vanishes on ] $-1,1[$. This implies that $\psi$ is of class $\mathcal{C}^{1+1 / 2}$ on $\Delta$ by equation (5.2) (see [73, 79]).

Step three: Geometric bootstrap. See Figure 7 . Let $v=(1,0)$ in $\mathbb{R}^{2}$ and consider the disc $\varphi^{c}$ defined on $\Delta^{+}$by

$$
\varphi^{c}(\zeta)=(\varphi(\zeta), d \varphi(\zeta)(v))
$$

The cluster set $C\left(\varphi^{c},\right]-1,1[)$ is contained in the smooth submanifold $T N$ of $T M$.

Lemma 5.1.2. If $N$ is a totally real submanifold in an almost complex manifold $(M, J)$ then $T N$ is a totally real submanifold in $\left(T M, J^{c}\right)$.

Proof of Lemma 5.1.2. Let $X \in T(T N) \cap J^{c}(T(T N))$. If $X=(u, v)$ in the trivialisation $T(T M)=T M \oplus T M$ then $u \in T N \cap J(T N)$, implying that $u=0$. Hence $v \in T N \cap J(T N)$, implying that $v=0$. Finally, $X=0$.

Applying Step two to $\varphi^{c}$ and $T N$ we prove that the first derivative of $\varphi$ with respect to $x(x+i y$ are the standard coordinates on $\mathbb{C})$ is of class $\mathcal{C}^{1+1 / 2}$ on $\left.\Delta^{+} \cup\right]-1,1[$. The $J$-holomorphicity equation (5.1) may be written as

$$
\frac{\partial \varphi}{\partial y}=J(\varphi) \frac{\partial \varphi}{\partial x}
$$

on $\left.\Delta^{+} \cup\right]-1,1\left[\right.$. Hence $\partial \varphi / \partial y$ is of class $\mathcal{C}^{1+1 / 2}$ on $\left.\Delta^{+} \cup\right]-1,1[$, meaning that $\varphi$ is of class $\mathcal{C}^{2+1 / 2}$ on $\left.\Delta^{+} \cup\right]-1,1$. We prove now that $\varphi$ is of class $\mathcal{C}^{3+1 / 2}$ on $\left.\Delta^{+} \cup\right]-1,1$. The reader will conclude, repeating the same argument that $\varphi$ is of class $\mathcal{C}^{\infty}$ on $\left.\Delta^{+} \cup\right]-1,1[$.

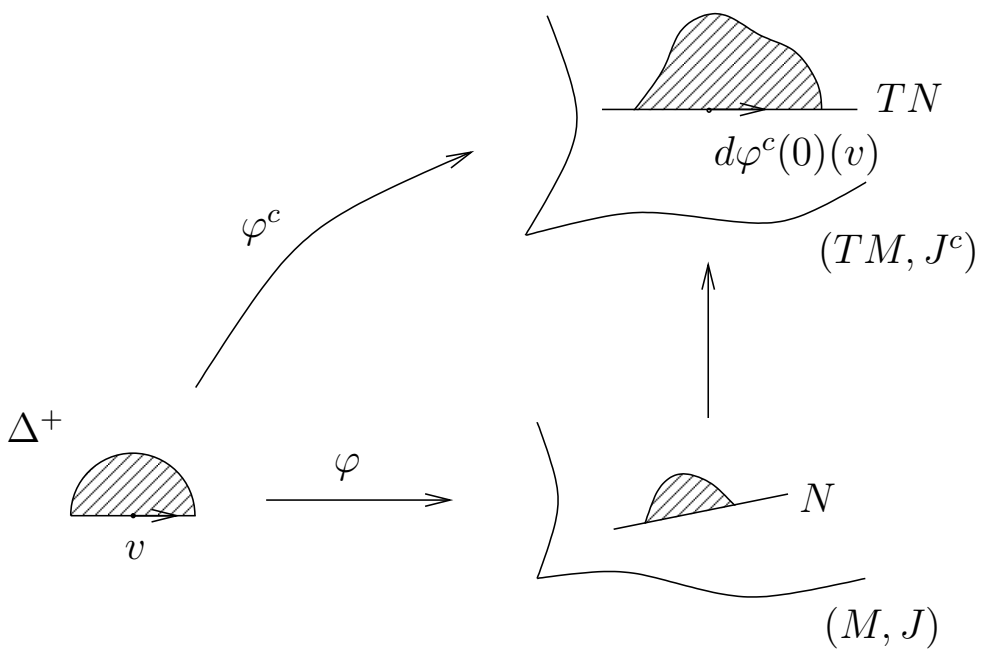

Figure 7

Replace now the data $(M, J)$ and $\varphi$ by $\left(T M, J^{c}\right)$ and $\varphi^{c}$ in Step three. The map ${ }^{2} \varphi^{c}$ defined on $\Delta^{+}$by ${ }^{2} \varphi^{c}(\zeta)=\left(\varphi^{c}(\zeta), d \varphi^{c}(\zeta)(v)\right)$ is ${ }^{2} J^{c}$-holomorphic on $\Delta^{+}\left({ }^{2} J^{c}\right.$ is the complete lift of $J^{c}$ to the second tangent bundle $T(T M)$. According to Step two, its first derivative 
$\partial\left({ }^{2} \varphi^{c}\right) / \partial x$ is of class $C^{1+1 / 2}$ on $\left.\Delta^{+} U\right]-1,1\left[\right.$. This means that the second derivatives $\frac{\partial^{2} \varphi}{\partial x^{2}}$ and $\frac{\partial^{2} \varphi}{\partial x \partial y}$ are $C^{1+1 / 2}$ on $\left.\Delta^{+} \cup\right]-1,1[$. Differentiating equation (5.1) with respect to $y$, we prove that $\frac{\partial^{2} \varphi}{\partial y^{2}}$ is $C^{1+1 / 2}$ on $\left.\Delta^{+} \cup\right]-1,1\left[\right.$ and so that $\varphi$ is $C^{3+1 / 2}$ on $\left.\Delta^{+} \cup\right]-1,1[$.

5.2. Behavior of pseudoholomorphic maps near totally real submanifolds. Let $\Omega$ be a domain in an almost complex manifold $(M, J)$ and $E \subset \Omega$ be a smooth $n$-dimensional totally real submanifold defined as the set of common zeros of the functions $r_{j}, j=1, \ldots, n$ smooth on $\Omega$. We suppose that $\bar{\partial}_{J} r_{1} \wedge \ldots \wedge \bar{\partial}_{J} r_{n} \neq 0$ on $\Omega$. Consider the "wedge" $W(\Omega, E)=$ $\left\{z \in \Omega: r_{j}(z)<0, j=1, \ldots, n\right\}$ with "edge" $E$. For $\delta>0$ we denote by $W_{\delta}(\Omega, E)$ the "shrinked" wedge $\left\{z \in \Omega: r_{j}(z)-\delta \sum_{k \neq j} r_{k}<0, j=1, \ldots, n\right\}$. The main goal of this Section is to prove the following

Proposition 5.2.1. Let $W(\Omega, E)$ be a wedge in $\Omega \subset(M, J)$ with a totally real $n$-dimensional edge $E$ of class $\mathcal{C}^{\infty}$ and let $f: W(\Omega, E) \rightarrow\left(M^{\prime}, J^{\prime}\right)$ be a $\left(J, J^{\prime}\right)$-holomorphic map. Suppose that the cluster set $C(f, E)$ is (compactly) contained in a $\mathcal{C}^{\infty}$ totally real submanifold $E^{\prime}$ of $M^{\prime}$. Then for any $\delta>0$ the map $f$ extends to $W_{\delta}(\Omega, E) \cup E$ as a $\mathcal{C}^{\infty}$-map.

We previously established this statement for a single $J$-holomorphic disc. The general case also relies on the ellipticity of the $\bar{\partial}$-operator. It requires an additional technique of attaching pseudoholomorphic discs to a totally real manifold which could be of independent interest.

Now we prove Proposition 5.2.1. Let $\left(h_{t}\right)_{t}$ be the family of $J$-holomorphic discs, smoothly depending on the parameter $t \in \mathbb{R}^{2 n}$, defined in Lemma 1.3.5. It follows from Lemma 3.3.5, applied to the holomorphic disc $f \circ h_{t}$, uniformly with respect to $t$, that there is a constant $C$ such that $\|d f(z)\| \| \leq \operatorname{dist}(z, E)^{-1 / 2}$ for any $z \in W_{\delta}(\Omega, E)$. This implies that $f$ extends as a Hölder $1 / 2$-continuous map on $W_{\delta}(\Omega, E) \cup E$.

It follows now from Theorem 5.1.1 that every composition $f \circ h_{t}$ is smooth up to $\partial \Delta^{+}$. Moreover, the direct examination of our argument shows that the $\mathcal{C}^{k}$ norm of the discs $f \circ h_{t}$ are uniformly bounded, for any $k$. Recall the separate smoothness principle (Proposition 3.1, [77]):

Proposition 5.2.2. Let $F_{j}, 1 \leq j \leq n$, be $\mathcal{C}^{\alpha}$ ( $\alpha>1$ noninteger) smooth foliations in a domain $\Omega \subset \mathbb{R}^{n}$ such that for every point $p \in \Omega$ the tangent vectors to the curves $\gamma_{j} \in F_{j}$ passing through $p$ are linearly independent. Let $f$ be a function on $\Omega$ such that the restrictions $f_{\mid \gamma_{j}, 1}, j \leq n$, are of class $\mathcal{C}^{\alpha-1}$ and are uniformly bounded in the $\mathcal{C}^{\alpha-1}$ norm. Then $f$ is of class $\mathcal{C}^{\alpha-1}$.

Using Lemma 1.3.5 we construct $n$ transversal foliations of $E$ by boundaries of Bishop's discs. Since the restriction of $f$ on every such curve satisfies the hypothesis of Proposition 5.2.2, $f$ is smooth up to E. This proves Proposition 5.2.1.

Let $\Gamma$ and $\Gamma^{\prime}$ be two totally real maximal submanifolds in almost complex manifodls $(M, J)$ and $\left(M^{\prime}, J^{\prime}\right)$. Let $W(\Gamma, M)$ be a wedge in $M$ with edge $\Gamma$. 
Proposition 5.2.3. If $F: W(\Gamma, M) \rightarrow M^{\prime}$ is $\left(J, J^{\prime}\right)$-holomorphic and if the cluster set of $\Gamma$ is contained in $\Gamma^{\prime}$ then $F$ extends as a $\mathcal{C}^{\infty}$ map up to $\Gamma$.

Proof of Proposition 5.2.3. In view of Proposition 5.1.1 the proof is classical (see [24]).

As a direct application of Proposition 5.2 .3 we obtain the following partial version of Fefferman's Theorem :

Corollary 5.2.4. Let $D$ and $D^{\prime}$ be two smooth relatively compact domains in real manifolds. Assume that $D$ admits an almost complex structure $J$ smooth on $\bar{D}$ and such that $(D, J)$ is strictly pseudoconvex. Let $f$ be a smooth diffeomorphism $f: D \rightarrow D^{\prime}$, extending as a $\mathcal{C}^{1}$ diffeomorphism (still called $f$ ) between $\bar{D}$ and $\bar{D}^{\prime}$. Then $f$ is a smooth $\mathcal{C}^{\infty}$ diffeomorphism between $\bar{D}$ and $\bar{D}^{\prime}$ if and only if the direct image $f_{*}(J)$ of $J$ under $f$ extends smoothly on $\bar{D}^{\prime}$ and $\left(D^{\prime}, f_{*}(J)\right)$ is strictly pseudoconvex.

Proof of Corollary 5.2.4. The cotangent lift $f^{*}$ of $f$ to the cotangent bundle over $D$, locally defined by $f^{*}:=\left(f,{ }^{t}(d f)^{-1}\right)$, is a $\left(\tilde{J}, \tilde{J}^{\prime}\right)$-biholomorphism from $T^{*} D$ to $T^{*} D^{\prime}$, where $J^{\prime}:=f_{*}(J)$. According to Proposition 1.5.2, the conormal bundle $\Sigma(\partial D)$ (resp. $\left.\Sigma\left(\partial D^{\prime}\right)\right)$ is a totally real submanifold in $T^{*} M$ (resp. $\left.T^{*} M^{\prime}\right)$. We consider $\Sigma(\partial D)$ as the edge of a wedge $W(\Sigma(\partial D), M)$ contained in $T D$. Then we may apply Proposition 5.2 .3 to $F=f^{*}$ to conclude.

5.3. Fefferman's mapping Theorem. Here we present one of the main results of our paper. This was obtained in the paper [34].

Theorem 5.3.1. Let $D$ and $D^{\prime}$ be two smooth relatively compact domains in real manifolds. Assume that $D$ admits an almost complex structure $J$ smooth on $\bar{D}$ and such that $(D, J)$ is strictly pseudoconvex. Then a smooth diffeomorphism $f: D \rightarrow D^{\prime}$ extends to a smooth diffeomorphism between $\bar{D}$ and $\bar{D}^{\prime}$ if and only if the direct image $f_{*}(J)$ of $J$ under $f$ extends smoothly on $\bar{D}^{\prime}$ and $\left(D^{\prime}, f_{*}(J)\right)$ is strictly pseudoconvex.

Theorem 5.3.1 is a consequence of Proposition 4.3.6. We recall that according to Proposition 1.5 .2 the conormal bundle $\Sigma_{J}(\partial D)$ of $\partial D$ is a totally real submanifold in the cotangent bundle $T^{*} M$. Consider the set

$$
S=\left\{(z, L) \in \mathbb{R}^{2 n} \times \mathbb{R}^{2 n}: \operatorname{dist}\left((z, L), \Sigma_{J}(\partial D)\right) \leq \operatorname{dist}(z, \partial D), z \in D\right\} .
$$

In a neighborhood $U$ of any totally real point of $\Sigma_{J}(\partial D)$, the set $\mathrm{S}$ contains a wedge $W_{U}$ with $\Sigma_{J}(\partial D) \cap U$ as totally real edge.

Then in view of Proposition 5.2.3 we obtain the following Proposition :

Proposition 5.3.2. There is a wedge $W_{U^{\prime}}$ contained in the wedge $W_{U}$ such that the map $f^{*}$ extends to $W_{U^{\prime}} \cup \Sigma(\partial D)$ as a $\mathcal{C}^{\infty}$-map.

Proposition 5.3 .2 implies immediately that $f$ extends as a smooth $\mathcal{C}^{\infty}$ diffeomorphism from $\bar{D}$ to $\bar{D}^{\prime}$ (see Figure 8 ). 


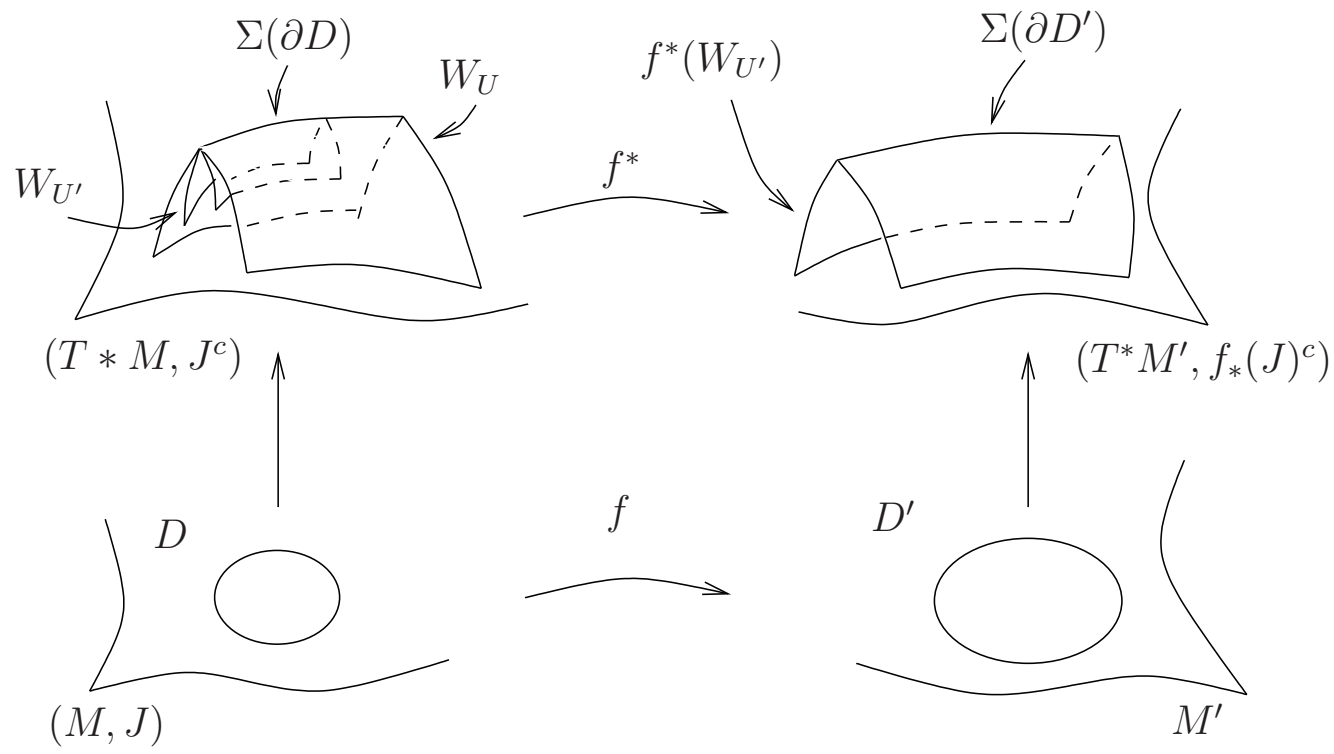

Figure 8

In this survey, we presented an overview of different results dealing with local analysis in almost complex manifolds, establishing some bases of the geometry of nonintegrable structures. We point out that there are many open questions concerning for instance the contact geometry (the contact properties of the Riemann map,...), the study of Monge-Ampère equations, or the links between almost complex analysis and symplectic topology. Our approach here may be considered as a necessary first step to study such questions.

\section{REFERENCES}

[1] Audin, M., Lafontaine, J., Holomorphic curves in symplectic geometry, Birkhäuser, Progress in Mathematics, 117, 1994.

[2] Balogh, Z., Leuenberger, Ch., Higher dimensional Riemann maps, Internat. J. Math. 9 (1998), 421-442.

[3] Barraud, J.F., Mazzilli, E., Regular type of real hyper-surfaces in (almost) complex manifolds, Math. Z. 248 (2004), 757-772.

[4] Bell, S., Lempert, L., A $\mathcal{C}^{\infty}$ Schwarz reflection principle in one and several complex variables, J. Differential Geom. 32-2 (1990), 899-915.

[5] Bennequin, D., Topologie symplectique, convexité holomorphe holomorphe et structures de contact [d'après Y. Eliashberg, D. Mc Duff et al.], Astérisque 189-190 (1990), 285-323.

[6] Berteloot, F., Attraction des disques analytiques et continuité höldérienne d'applications holomorphes propres, Topics in complex analysis (Warsaw, 1992), 91-98, Banach Center Publ., 31, Polish Acad. Sci., Warsaw, 1995.

[7] Berteloot, F., Principe de Bloch et estimations de la métrique de Kobayashi dans les domaines de $\mathbb{C}^{2}$, J. Geom. Anal., 13-1 (2003), 29-37.

[8] Berteloot, F., Coeuré, G., Domaines de $\mathbb{C}^{2}$, pseudoconvexes et de type fini ayant un groupe non compact d'automorphismes, Ann. Inst. Fourier 41 (1991), 77-86.

[9] Bland, J., Contact geometry and CR structures on $\mathbb{S}^{3}$, Acta Math. 172 (1994), 1-49.

[10] Bland, J., Duchamp, T., Moduli for pointed convex domains, Invent. Math. 104 (1991), 61-112. 
[11] Bland, J., Duchamp, T., Kalka, M., A characterization of $\mathbb{C P}^{n}$ by its automorphism group, Lecture Notes in Math. 1268 (1987), 60-65.

[12] Bu, S., Schachermayer, W., Approximation of Jensen measures by image measures under holomorphic functions and applications, Trans. AMS. 331 (1992), 585-608.

[13] Cartan, É., Sur la géométrie pseudo-conforme des hypersurfaces de l'espace de deux variables complexes, I, Annali di Mat. 11 (1932), 17-90.

[14] Cegrell, U., Sur les ensembles singuliers impropres des fonctions plurisousharmoniques C.R. Acad. Sci. Paris, 281(1975), 905-908.

[15] Cerne, M., Stationary discs of fibrations over the circle, Internat. J. Math. 6 (1995), 805-823.

[16] Chern, S. S., Moser, J. K. Real hypersurfaces in complex manifolds, Acta Math. 133 (1974), 219271.

[17] Chirka, E., Regularity of boundaries of analytic sets, Math. USSR Sb. 45(1983), 291-336.

[18] Chirka, E., Introduction to the almost complex analysis, Lecture notes (2003).

[19] Chirka, E., Personal communication.

[20] Chirka, E., Coupet, B., Sukhov,A., On boundary regularity of analytic discs, Mich. Math. J. 46 (1999), 271-279.

[21] Clancey, K., Gohberg, I., Factorization of matrix functions and singular integral operators, Birkhauser, Basel, Boston, Stuttgart, 1981.

[22] Coupet, B., Precise regularity up to the boundary of proper holomorphic mappings Ann. Scuola Norm. Sup. Pisa 20 (1993), 461-482.

[23] Coupet, B. Gaussier, H., Sukhov, A., Riemann maps in almost complex manifolds, Ann. Sc. Norm. Super. Pisa Cl. Sci. 5 (2003), 761-785.

[24] Coupet, B. Gaussier, H., Sukhov, A., Fefferman's mapping theorem on almost complex manifolds in complex dimension two, Math. Z. 250 (2005), 59-90.

[25] Debalme, R., Kobayashi hyperbolicity of almost complex manifolds, preprint of the University of Lille, IRMA 50 (1999), math.CV/9805130.

[26] Debalme, R., IVAshkovich, S., Complete hyperbolicit neiborhoods in almost complex surfaces, Int. J. Math. 12 (2001), 211-221.

[27] Diederich, K., Fornaess, J.E., Proper holomorphic maps onto pseudoconvex domains with real analytic boundary, Ann. Math. 110 (1979), 575-592.

[28] Diederich, K, Sukhov, A., Diffeomorphisms of Stein structures, math. CV/0603416, to appear in Journ. Geom. Analysis.

[29] Diederich, K, Sukhov, A., Plurisubharmonic exhaustion functions and almost complex Stein structures, mat. CV/0603417.

[30] Edgar, G.A., Complex martingale convergence, Lecture Notes in Math. 1116 (1985), 38-59.

[31] Fefferman, Ch., The Bergman kernel and biholomorphic mappings of pseudoconvex domains, Invent. math. 26 (1974), 1-65.

[32] Forstneric, F., An elementary proof of Fefferman's theorem, Exposit. Math. 10 (1992), 135-149.

[33] Gaussier, H., Sukhov, A., Estimates of the Kobayashi metric in almost complex manifolds, ArXiv math.cv/0307334, Bull. Soc. Math. France 133 (2005), 259-273.

[34] Gaussier, H., Sukhov, A., On the geometry of model almost complex manifolds with boundary, math. CV/0412095, to appear in Math. Z.

[35] Globevnik, J., Perturbation by analytic discs along maximal real submanifolds of $\mathbb{C}^{N}$, Math. Z. 217 (1994), 287-316.

[36] Globevnik, J., Perturbing analytic discs attached to a maximal totally real submanifolds of $\mathbb{C}^{n}$, Indag. Math. 7 (1996), 37-46.

[37] Graham, I., Boundary behavior of the Carathéodory and Kobayashi metrics on strongly pseudoconvex domains in $C^{n}$ with smooth boundary, Trans. Amer. Math. Soc. 207 (1975), 219-240.

[38] Grauert, H., Lieb, I., Das Ramirezsche Integral und die Lösung der Gleichung $\bar{\partial} f=\alpha$ im Bereich der beschränkten Formen, (German) Rice Univ. Studies 56 (1970), 29-50.

[39] Gromov, M., Pseudoholomorphic curves in symplectic manifolds, Invent. Math. 82-2 (1985), 307-347. 
[40] Hamilton, R., Deformation of complex structures on manifolds with boundary. I. The stable case. J. Differential Geometry 12 (1977), 1-45.

[41] Henkin, G., Integral representation of functions in strongly pseudoconvex regions, and applications to the $\bar{\partial}$-problem (Russian) Mat. Sb. (N.S.) 82 (124) (1970), 300-308.

[42] Hofer, H., Pseudoholomorphic curves in symplectizations with applications to the Weinstein conjecture in dimension three. Invent. Math. 114 (1993), 515-563.

[43] Ishihara, K. Yano, Tangent and cotangent bundles: differential geometry, Pure ans Applied Mathematics, No. 16. Marcel Dekker Inc, New York, 1973.

[44] Ivashkovish, S., Rosay, J.P., Schwarz-type lemmas for solutions of $\bar{\partial}$-inequalities and complete hyperbolicity of almost complex manifolds, Preprint.

[45] Ivashkovish, S., Shevchishin, V., Reflection principle and J-complex curves with boundary on totally real immersions, Comm. in Contemp. Math. 4-1 (2002), 65-106.

[46] Karpova, N.G., On the removal of singularoties of plurisubharmonic functions, Math. Notes. 49 (1991) English. Trans. Math. USSR Sb. 63 (1989), 252-256.

[47] Kobayashi, S., Hyperbolic complex spaces. Grundlehren der Mathematischen Wissenschaften [Fundamental Principles of Mathematical Sciences], 318. Springer-Verlag, Berlin, 1998.

[48] Kobayashi, S., Almost complex manifolds and hyperbolicity. Dedicated to Shiing-Shen Chern on his 90th birthday. Results Math. 40 (2001), no. 1-4, 246-256.

[49] Kerzman, N., Rosay, J.-P., Fonctions plurisousharmoniques d'exhaustion bornées et domaines taut. (French) [Bounded plurisubharmonic exhaustion functions and taut domains], Math. Ann. 257 (1981), no. $2,171-184$.

[50] Kruglikov, B.S., Existence of close pseudoholomorphic disks for almost complex manifolds and their application to the Kobayashi-Royden pseudonorm. (Russian) Funktsional. Anal. i Prilozhen. 33 (1999), no. 1, 46-58, 96; translation in Funct. Anal. Appl. 33 (1999), no. 1, 38-48.

[51] Kruglikov, B.S., Deformation of big pseudoholomorphic discs and application to the Hanh pseudonorm arXiv: math. CV/0304166 v1 14.04.2003.

[52] Lempert, L., La métrique de Kobayashi et la representation des domaine sur la boule, Bull. Math. Soc. France 109 (1981), 427-474.

[53] Lempert, L., Solving the degenerate complex Monge-Ampère equation with one concentrated singularity, Math. Ann. 263 (1983), 515-532.

[54] LemperT, L., A precise result on the boundary regularity of biholomorphic mappings, Math. Z. 193 (1986), 559-579.

[55] Lempert, L., Holomorphic invariants, normal forms and moduli space of convex domains, Ann. of Math. 128 (1988), 47-78.

[56] LEMPeRT, L., Erratum: A precise result on the boundary regularity of biholomorphic mappings, Math. Z. 206 (1991), 501-504.

[57] Lempert, L., Szöke, R., The tangent bundle of an almost complex manifold, Canad. Math. Bull. 44 (2001), 70-79.

[58] Libermann, P., Problèmes d'équivalence relatifs à une structure presque complexe sur une variété à quatre dimensions, Acad. Roy. Belgique Bull. Cl. Sci. (5) 36 (1950), 742-755.

[59] McDuff, D., Salamon, D., J-holomorphic curves and symplectic topology, American Mathematical Society Colloquium Publications, 52. Providence, RI, 2004. xii+669 pp.

[60] Newlander, A., Nirenberg, L., Complex analytic coordinates in almost complex manifolds, Ann. of Math. (2) 65 (1957), 391-404.

[61] Nijenhuis, A., Woolf, W., Some integration problems in almost-complex and complex manifolds, Ann. Math. 77 (1963), 429-484.

[62] Nirenberg, L., Webster, S., YAng, P., Local boundary regularity of holomorphic mappings, Comm. Pure Applied Math. 33 (1980), 305-338.

[63] Pang, M.Y., Smoothness of the Kobayashi metric of non-convex domains, Int. J. Math. 4 (1993), 953-987. 
[64] Pflug, P., Ein Fortsetzungsatz fur plurisubharmonische Funktionen uber reell-2-kodimensionale Flachen Arch. Math. (Basel). 33(1979/80), 559-663.

[65] Pinchuk, S., A boundary uniqueness theorem for holomorphic functions of several complex variables Matth. Notes 15(1974), 116-120.

[66] Ransford, Th., Potential theory in the complex plane, Cambridge Univ. Press, 1995.

[67] Pinchuk, S., The scaling method and holomorphic mappings, Several complex variables and complex geometry, Part 1 (Santa Cruz, CA, 1989), 151-161, Proc. Sympos. Pure Math., 52 Part 1, Amer. Math. Soc., Providence, RI, 1991.

[68] Pinchuk, S., Khasanov, S., Asymptotically holomorphic functions and their applications, Math. USSR Sb. 62 (1989), 541-550.

[69] Royden, H.L., Remarks on the Kobayashi metric, Lecture Notes in Mathematics 185, Springer-Verlag, 1970, pp.125-137.

[70] Semmes, S., A generalization of Riemann mappings and geometric structures on a space of domains in $\mathbb{C}^{n}$, Mem. Amer. Math. Soc. 98 (1992), vi+98pp.

[71] Shiffman, B., Extension of positive line bundles and meromorphic maps Invent. math. 15(1972), 332-347.

[72] Sibony, N., A class of hyperbolic manifolds, Ann. of Math. Stud., 100, pp.91-97, Princeton Univ. Press, Princeton, NJ, 1981.

[73] Sikorav, J.-C., Some properties of holomorphic curves in almost complex manifolds in "Holomorphic curves and symplectic geometry", Ed. M.Audin, J.Lafontaine, Birkhauser, (1994), 165-189.

[74] SpIRo, A. Total reality of the conormal bundle of a real hypersurface in an almost complex manifold, Preprint, December 2003.

[75] Spiro, A., Sukhov A. An existence theorem for stationary discs in almost complex manifolds, math. CV/0502121.

[76] Spiro, A., Trapani, S., Eversive maps of bounded convex domains in $\mathbb{C}^{n+1}$, J. Geom. Anal. 12 (2002), 695-715.

[77] Tumanov, A., Analytic discs and the regularity of CR mappings in higher codimension, Duke Math. J. 76 (1994), 793-807.

[78] Tumanov, A., Extremal discs and the regularity of CR mappings in higher codimension, Amer. J. Math. 123 (2001), 445-473.

[79] VeKuA, N.P. Systems of singular integral equations, Nordholf, Groningen 1967.

[80] Webster, S. On the reflection principle in several complex variables, Proc. Amer. Math. Soc. 71 (1978), 26-28.

[81] Webster, S. A new proof of the Newlander-Nirenberg theorem, Math. Z. 201 (1989), 303-316.

[82] Yano, K., Ishinara, Sh., Tangent and cotangent bundles, Marcel Dekker NY 1973.

$\begin{array}{lll}\text { Bernard Coupet } & \text { Hervé Gaussier } & \text { Alexandre Sukhov } \\ \text { C.M.I. } & \text { I.M.J. } & \text { U.S.T.L. } \\ \text { 39, Rue Joliot-Curie } & \text { 4, PlaCe Jussieu } & \text { Cité Scientifique } \\ \text { 13453 Marseille Cedex 13 } & 75252 \text { Paris Cedex } & 59655 \text { Villeneuve D'AscQ Cedex } \\ \text { coupet@cmi.univ-mrs.fr } & \text { gaussier@math.jussieu.fr } & \text { sukhov@agat.univ-lille1.fr }\end{array}$

